\title{
Tópicos em Métodos de Ponto Proximal
}

\author{
Paulo José da Silva e Silva
}

TESE APRESENTADA

$\mathrm{AO}$

INSTITUTO DE MATEMÁTICA E ESTATÍSTICA

DA

UNIVERSIDADE DE SÃO PAULO

PARA

OBTENÇÃO DO GRAU DE

DOUTOR EM MATEMÁTICA APLICADA

Área de Concentração: Otimização e Controle

Orientador: Prof. Dr. Carlos Humes Júnior

O aluno recebeu apoio da FAPESP - Processo 96/09939-0

São Paulo, Dezembro 2000 


\section{Tópicos em Métodos de Ponto Proximal}

Este exemplar corresponde à redação final da tese devidamente corrigida e defendida por Paulo José da Silva e Silva e aprovada pela comissão julgadora.

São Paulo, dezembro de 2000.

Banca Examinadora:

Prof. Dr. Carlos Humes Junior (orientador)

IME/USP

Prof. Dr. Clóvis Caesar Gonzaga

UFSC

Prof. Dr. Alfredo Noel Iusem

IMPA

Prof. Dr. José Mario Martinez Perez

IMECC/UNICAMP

Profa. Dra. Yoshiko Wakabayashi

IME/USP 
A meus avós João, Antônia, Francisco e Rita 


\section{Agradecimentos}

Uma lista como esta não tem fim...

Aos meus pais, pelo apoio e por me ensinarem, ainda pequeno, a admirar a beleza das idéias.

A minha noiva Silvina, pela paciência, atenção, carinho e, sobretudo, por ser a pessoa que é.

Aos meu irmão, Francisco, que me acompanhou estes últimos anos.

Ao Carlos, pela já longa cumplicidade na Matemática e fora dela.

Aos meus companheiros de Matemática, com quem tive o privilégio de trabalhar, em particular meus co-autores: Marcelo, Benar e Eckstein.

Ao Prof. Iusem, pelo belo curso que me apresentou os métodos proximais e pelo constante apoio em minha curta vida acadêmica.

Ao meu professor do segundo grau, Valdener, por ter me ensinado a me encantar com a Matemática.

Aos meus amigos que, graças a Deus, são muitos: Caputi, Raul, Cibele, Pil, Daniel, Liane, Fábio, Mané, Sônia, Prof. Barone, Possani, Lupa, Jack, Sô, Carine, Luís, Lílian, Rodrigo, Sílvia, Laura, Jerusa, Cláucio e tantos outros com quem dividi minhas horas. 


\section{Abstract}

This thesis deals with proximal point methods and their use to solve variational inequalities and convex optimization problems.

We present two new regularizations families and the respective proximal methods. The first family, simple in concept, is based on translations of strict convex functions. The second family contains a broad class of coercive regularizations, extending recent results in the literature. In particular, we extend the double regularizations presented by Auslender et al. to a wide class that contains Bregman distances and we succeed to prove that these regularizations may be used to solve variational inequalities with maximal monotone operators that may not be para-monotone. We also explore the relationship of proximal and multiplier methods.

Finally, we present some new criteria to accept approximate solutions of the unconstrained problems that have to be solved by multiplier methods. 


\section{Índice}

1 Introdução e Problemas 1

1.1 Introdução . . . . . . . . . . . . . . . . . . . 1

1.2 Os Problemas . . . . . . . . . . . . . . . . . 3

1.3 Métodos Proximais . . . . . . . . . . . . . . 5

2 MPP, Descida e Multiplicadores 7

2.1 MPP inexato baseado em descida . . . . . . . . . . . . . 7

2.2 Conexão com métodos de multiplicadores . . . . . . . . . . . . 15

3 Distâncias Baseadas em Translação 24

3.1 Métodos de ponto proximal e funções estritamente convexas . . . . . . 24

3.2 Uma versão inexata do $\phi$-MPP . . . . . . . . . . . . . . . . 28

3.3 Métodos de multiplicadores . . . . . . . . . . . . . 30

4 Distâncias Coercivas $\quad 34$

4.1 Regularizações Coercivas Separáveis . . . . . . . . . . . . . . . . . 34

4.2 O Método Proximal com Distâncias Coercivas Separáveis . . . . . . . . 40

4.3 Distância Coercivas Separáveis Aplicadas à Otimização . . . . . . . . . . 44

5 Regularizações Duplas $\quad 50$ 
5.1 Uma Desigualdade Interessante . . . . . . . . . . . . . . . . . 51

5.2 Análise de Convergência . . . . . . . . . . . . . . . . . 54

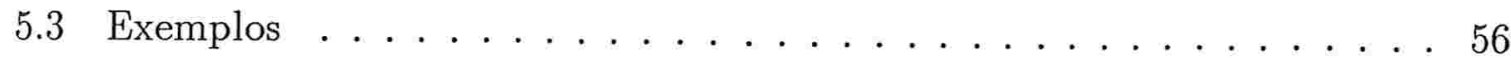

5.4 Penalidades e Métodos de Multiplicadores . . . . . . . . . . . . 61

6 Resolução Aproximada em Métodos de Multiplicadores $\quad 65$

6.1 Métodos de Multiplicadores com Distâncias Coercivas . . . . . . . . . . 65

6.2 O Método Híbrido de Solodov e Svaiter . . . . . . . . . . . . . 69

$\begin{array}{lll}7 \text { Conclusão } & 74\end{array}$

A Existência de zeros de desigualdades variacionais regularizadas $\quad 76$

B Conexões entre distâncias de Bregman e $\varphi$-divergências $\quad 81$

C Tabelas dos Testes Numéricos $\quad 86$

C.1 Mult. e Distâncias Coercivas . . . . . . . . . . . . . . . . . 87

C.2 Método Híbrido Projeção-Lagrangiano Aumentado Proximal . . . . . . . 93 


\section{Lista de Figuras}

2.10 ângulo $\theta \ldots \ldots \ldots \ldots \ldots$

2.2 O passo de projeção . . . . . . . . . . . . . . . . . 14

2.3 Penalidade $P(u)=2 u+\sqrt{u^{2}+1} \ldots \ldots \ldots \ldots$

3.1 Exemplos de penalidade . . . . . . . . . . . . . . 31

5.1 Limites para as derivadas de uma regularização dupla . . . . . . . . . . 52

5.2 Limites para derivada de uma penalidade . . . . . . . . . . . . 63

B.1 Mudança de escala atuando na dist. de Bregman e na $\varphi$-divergência baseadas em $h(x)=x \log \left(e^{x}-1\right) \ldots \ldots . \ldots . \ldots 82$

B.2 Mudança de escala atuando na dist. de Bregman e na $\varphi$-divergência baseadas em $h(x)=\operatorname{dilog}\left(e^{x}\right)+x \log \left(e^{x}-1\right) \ldots \ldots 83$

B.3 Mudança de escala atuando na dist. de Bregman e na $\varphi$-divergência baseadas em $h(x)=x^{2}-\sqrt{x} \ldots \ldots . \ldots . \ldots . \ldots 83$ 


\section{Lista de Tabelas}

C.1 Conjunto de 130 problemas com restrições de desigualdades da coleção do CUTE/mastsif_small [10] que serviu de base para os testes. . . . . . 86

C.2 Problemas resolvidos pela versão exata do Algoritmo 4.3.5 . . . . . . 87

C.3 Problemas resolvidos pela versão inexata do Algoritmo 4.3.5 . . . . . 87

C.4 Problemas usados no teste comparativo do Algoritmo 4.3.5 . . . . . . 88

C.5 Comparação entre as versões exata e inexata do Algoritmo 4.3 .5 . . . . . 89

C.6 Comparação entre as versões exata e inexata do Algoritmo 4.3.5 . . . . . 90

C.7 Comparação entre as versões exata e inexata do Algoritmo 4.3 .5 . . . . . 91

C.8 Comparação entre as versões exata e inexata do Algoritmo 4.3 .5 . . . . . 92

C.9 Problemas resolvidos pela versão exata do algoritmo Híbrido . . . . . . . 93

C.10 Problemas resolvidos pela versão inexata do algoritmo Híbrido . . . . . . 93

C.11 Problemas usados no teste comparativo do algoritmo Híbrido . . . . . . . 94

C.12 Comparação entre as versões exata e inexata do algoritmo Híbrido . . . . 95

C.13 Comparação entre as versões exata e inexata do algoritmo Híbrido . . . . 96

C.14 Comparação entre as versões exata e inexata do algoritmo Híbrido . . . . 97

C.15 Comparação entre as versões exata e inexata do algoritmo Híbrido . . . . 98 


\section{Notações e Definições}

$\mathrm{a} \leq \mathrm{b}$ : $\mathrm{O}$ vetor $\mathrm{a} \in \mathbb{R}^{\mathrm{n}}$ é menor ou igual $\mathrm{a} b \in \mathbb{R}^{\mathrm{n}}$ coordenada à coordenada. Se a relação for estrita (para todas as coordenadas) escrevemos $a<b$.

$a_{+}$: Vetor, de mesma dimensão de $a$, cujas coordenadas são iguais a $\max \left(0, a_{i}\right), \forall i$. Analogamente, $a_{-}$denota o vetor com coordenadas iguais a $\min \left(0, a_{i}\right), \forall i$.

$a_{i}: a$-ésima coordenada do vetor $a \in \mathbb{R}^{n}$.

$\operatorname{int} A$ : Interior do conjunto $A \subset \mathbb{R}^{n}$.

ri $A$ : Interior relativo do conjunto $A \subset \mathbb{R}^{n}$ (interior de $A$ com relação à "maior" variedade afim que o contém).

$\left\{x^{k}\right\}_{\mathcal{A}}, \mathcal{A} \subset \mathbb{N}$ : Uma seqüência com índices em $\mathcal{A}$. Se $\mathcal{A}=\mathbb{N}$, usamos a notação $\left\{x^{k}\right\}$. Caso a seqüência seja de números reais (e não de vetores), usaremos a notação $\left\{a_{k}\right\}$.

$x^{k} \rightarrow_{\mathcal{A}} \bar{x}:$ A seqüência $\left\{x^{k}\right\}_{\mathcal{A}}$ converge para $\bar{x}$. Mais uma vez podemos omitir o conjunto $\mathcal{A}$, caso este coincida com o $\mathbb{N}$.

$f: A \rightarrow B$ : Função que associa a cada elemento do conjunto $A$ um elemento de $B$.

$\mathrm{T}: \mathrm{A} \rightrightarrows \mathrm{B}$ : Operador que associa a cada elemento de $A$ um subconjunto de $\mathrm{B}$.

dom f: Domínio efetivo de f. Se $f: A \rightarrow \overline{\mathbb{R}}$ é uma função, dom $f$ é o conjunto de pontos $x$ de $A$ tais que $f(x)<+\infty$. Se $f: A \rightrightarrows B$ é um operador, dom $f$ é o conjunto de pontos $x$ de $A$ tais que $f(x) \neq \emptyset$.

Uma função $f: A \rightarrow B$ é fechada ou semi-contínua inferior (sci) se, dada $\left\{x^{k}\right\} \subset A$, $x \rightarrow \bar{x} \in A$, então $\liminf \operatorname{in}_{k \rightarrow \infty} f\left(x^{k}\right) \geq f(\bar{x})$. 
Um operador $\mathrm{T}: \mathrm{A} \rightrightarrows \mathrm{B}$ é semi-continuo exterior se, $\left\{x^{k}\right\} \subset A, x \rightarrow \bar{x} \in A$ e $\left\{y^{k}\right\} \subset B$, $y^{k} \in T\left(x^{k}\right)$ e $y^{k} \rightarrow \bar{y} \in B$, então $\bar{y} \in T(\bar{x})$.

Uma função $f: \mathbb{R}^{n} \rightarrow \overline{\mathbb{R}}$ é dita própria se ela não vale $-\infty$ em nenhum ponto $x \in \mathbb{R}^{n}$ e se $f$ for finita em pelo menos um ponto de seu domínio.

$\mathrm{C} \subset \mathbb{R}^{n}$ é chamado de conjunto convexo se, para todo $\zeta \in[0,1]$ e todos $c_{1}, \mathrm{c}_{2} \in \mathrm{C}$, $\zeta c_{1}+(1-\zeta) c_{2} \in C$.

Uma função $f: \mathbb{R}^{n} \rightarrow \overline{\mathbb{R}}$ é dita convexa se sua epígrafe, epi $f=\left\{(x, \alpha) \in \mathbb{R}^{n} \times \mathbb{R} \mid\right.$ $f(x) \leq \alpha\}$, for um conjunto convexo.

A lista de definições e resultados associados a conjuntos e funções convexas é muito extensa. Duas ótimas referêcias são [37, 41].

Se $C \in \mathbb{R}^{n}$ é um conjunto cujo fecho é convexo, chamamos de cone de recessão de $C$, denotato por $C^{\infty}$, o cone composto por todas os vetores $d \in \mathbb{R}^{n}$ tais que

$$
\forall x \in \operatorname{riC}, \forall \alpha \geq 0, \quad x+\alpha d \in C .
$$

Se $f: \mathbb{R}^{n} \rightarrow(-\infty, \infty]$ é uma função convexa, fechada e própria, tomemos $\bar{x} \in$ $\operatorname{dom} f$. A função $f^{\infty}: \mathbb{R}^{n} \rightarrow(-\infty, \infty]$, dada por

$$
f^{\infty}(d) \stackrel{\text { def }}{=} \lim _{t \rightarrow \infty} \frac{f(\bar{x}+t d)-f(\bar{x})}{t}
$$

é conhecida como função de recessão de f. $^{1}$

$\delta_{A}: \mathbb{R}^{n} \rightarrow(-\infty, \infty], A \subset \mathbb{R}^{n}:$ Denota a função indicadora de $A$, ou seja, a função que associa a cada $x \in \mathbb{R}^{n}$ o valor 0 , se $x \in A$, e $+\infty$, caso contrário.

Um operador $\mathrm{T}: \mathbb{R}^{\mathrm{n}} \rightrightarrows \mathbb{R}^{\mathrm{n}}$ é monótono se

$$
\forall x_{1}, x_{2} \in \mathbb{R}^{n}, \forall y_{1} \in T\left(x_{1}\right), \forall y_{2} \in T\left(x_{2}\right),\left\langle y_{1}-y_{2}, x_{1}-x_{2}\right\rangle \geq 0
$$

T é monótono maximal se ele não puder ser estendido preservando sua monotonicidade.

É impossível apresentar aqui todos os resultados associados a estes operadores. Uma ótima referência é [41, Capítulo 12].

\footnotetext{
${ }^{1}$ As noções de cone e função de recessão aparecem na literatura com nomes variados. Por exemplo, em [37] temos recession cone e recession function, já em [23, 24] encontramos asymptotic cone e asymptotic function. Por fim, em [41] usa-se horizon cone e horizon function. Optamos por basear nossa tradução em [37] por considerarmos que este livro é a principal referência da área de Análise Convexa em espaços de dimensão finita. Porém mantemos a notação de [41, Capítulo 3], por ser, ao nosso ver, mais sugestiva.
} 
Seja $f: \mathbb{R}^{n} \rightarrow \overline{\mathbb{R}}$. Um subgradiente de $f$ em $\bar{x}$ é um vetor $\gamma \in \mathbb{R}^{n}$, tal que

$$
\forall x \in \mathbb{R}^{n}, f(x) \geq f(\bar{x})+\langle\gamma, x-\bar{x}\rangle .
$$

$O$ operador que associa a todo ponto $x \in \mathbb{R}^{n}$ o conjunto dos subgradientes de $f$ em $x$ é chamado de subdiferencial de $f$ e denotado por $\partial f$.

Seja $f: \mathbb{R}^{n} \rightarrow \overline{\mathbb{R}}$. A função $f^{*}: \mathbb{R}^{n} \rightarrow \overline{\mathbb{R}}$, definida por

$$
\forall y \in \mathbb{R}^{n}, f^{*}(y) \stackrel{\text { def }}{=} \sup _{x}\{\langle y, x\rangle-f(x)\}
$$

é conhecida como função convexo conjugada a $\mathrm{f}$.

De forma análoga, a função $f_{*}: \mathbb{R}^{n} \rightarrow \overline{\mathbb{R}}$, definida por

$$
\forall y \in \mathbb{R}^{n}, f_{*}(y) \stackrel{\text { def }}{=} \inf _{x}\{\langle y, x\rangle-f(x)\}
$$

é conhecida como função côncova conjugada $a \mathrm{f}$.

Uma seqüência $\left\{x^{k}\right\} \subset \mathbb{R}^{n}$ é Fejér monótona com relação a um conjunto $A$ se

$$
\forall a \in A, \forall k \in \mathbb{N}, \quad\left\|x^{k+1}-a\right\| \leq\left\|x^{k}-a\right\|
$$

Dizemos que um vetor a é normal a um conjunto convexo $C$ em um ponto $\bar{x} \in C$ se a não forma um ângulo agudo com nenhum segmento de $C$ que tenha $\bar{x}$ como um dos extremos:

$$
\forall x \in C,\langle x-\bar{x}, a\rangle \leq 0 .
$$

$\mathrm{O}$ conjunto de todos os vetores normais é chamado de cone normal a $\mathrm{C}$ em $\bar{\chi}$. Este cone é sempre convexo.

Seja $f: \mathbb{R}^{n} \rightarrow \overline{\mathbb{R}}$ e $A \subset \mathbb{R}^{n}$. O conjunto de todos os pontos de mínimo (máximo) de $f$ em $A$ será denotado por

$$
\underset{x \in A}{\operatorname{Argmin}}\{f(x)\} \quad(\underset{x \in A}{\operatorname{Argmax}}\{f(x)\}) .
$$

Caso este conjunto contenha um único elemento, vamos denotar este ponto de mínimo (máximo) por

$$
\underset{x \in \mathcal{A}}{\operatorname{argmin}}\{f(x)\} \quad(\underset{x \in \mathcal{A}}{\operatorname{argmax}}\{f(x)\}) .
$$




\section{Introdução e Problemas}

\subsection{Introdução}

A década de 90 testemunhou uma grande atividade relacionada a métodos de ponto proximal aplicados à otimização convexa e a problemas de desigualdades variacionais, bem como ao estudo de sua conexão com métodos de multiplicadores. Nosso trabalho insere-se nesta tradição e objetiva apresentar algumas contribuições à teoria de pontos proximais.

Nosso envolvimento nesta área iniciou-se no mestrado, quando, baseados em idéias de separadores, analisamos alguns métodos proximais, sob o ponto de vista de convergência de subseqüências. A extensão destes resultados durante o primeiro ano de nosso doutorado e a iteração com os pareceristas do artigo [25] convenceram-nos a usar diretamente o separador implícito nestes métodos: a queda da função objetivo.

Esta ferramenta foi então aplicada a várias extensões, descritas abaixo. Mais uma vez, uma análise aprofundada das técnicas desenvolvidas permitiu o estudo de problemas sem a presença de função objetivo, levando-nos à investigação de problemas de desigualdades variacionais com operadores monótonos maximais.

Introduzimos duas novas classes de funções de regularização que permitem provar a convergência, no sentido de subseqüências, dos respectivos métodos proximais. A primeira classe, bastante simples, baseia-se em translações de funções estritamente convexas. A segunda consiste de uma ampla gama de regularizações coercivas que estende resultados recentes de Ben-Tal e Zibulevsky [6] e de Auslender, Teboulle e Ben-Tiba [4, 5]. Em particular, analisamos o conceito de regularizações duplas em um contexto que engloba distâncias de Bregman, mostrando que é possível usá-las para resolver problemas de desigualdade variacional com operadores que não são, necessariamente, para-monótonos. Estudamos também a ligação destes métodos com métodos 
de multiplicadores.

No caso de otimização, os métodos que propomos apresentam um critério de aceitação de soluções aproximadas dos sub-problemas proximais baseados nas condições propostas por Solodov e Svaiter em [43, 44], mas que não dependem de um passo extragradiente para garantir a otimalidade dos pontos de acumulação.

O restante do trabalho é dividido da seguinte forma:

O final do Capítulo 1 descreve os problemas que desejamos resolver e introduz um modelo simples de métodos proximais.

O Capítulo 2 inicia-se com uma nova análise de convergência do método híbrido proposto por Solodov e Svaiter em [43]; baseada em limites para queda da função objetivo a cada passo. A prova apresentada é bastante simples, mas já contém as idéias principais que vão permear a análise dos algoritmos propostos nos capítulos seguintes. Além disto, a Seção 2.2 contém a teoria necessária para relacionar métodos proximais e métodos de multiplicadores.

O Capítulo 3 introduz métodos proximais baseados em translações de funções estritamente convexas. Analisa também os métodos de multiplicadores que podemos derivar a partir desta classe de regularizações, estendendo a classe de penalidades $\mathrm{P}_{\mathrm{E}}^{+}$descrita por Bertsekas em [8].

O Capítulo 4 apresenta uma nova classe de distâncias coercivas e analisa a convergência de um modelo de algoritmo proximal baseado nesta classe. Mostramos que é possível construir exemplos de nossos termos coercivos a partir de $\varphi$-divergências e distâncias de Bregman, usando uma técnica que denominamos mudança de escala. Em seguida, este modelo é aplicado ao caso de otimização, resultando em uma generalização parcial de [5].

O Capítulo 5 estende a idéia de regularizações duplas descrita em [4, 5]. Esta interessante classe de distâncias generalizadas permite recuperar a Fejér monotonicidade da seqüência proximal com relação ao conjunto de soluções de um problema de desigualdade variacional.

O Capítulo 6 discute técnicas para permitir a solução aproximada dos sub-problemas de métodos de multiplicadores. Alguns experimentos numéricos preliminares são apresentados.

No Capítulo 7, apresentamos brevemente algumas linhas de desenvolvimento futuro.

O Apêndice B explora, rapidamente, a correlação entre $\varphi$-divergências e distâncias de Bregman quando aplicamos a mudança de escala. Descrevemos como várias regularizações podem ser obtidas, ao mesmo tempo, de membros destas duas classes. 


\subsection{Os Problemas}

Esta seção tem como objetivo servir de ponto central para a definição e referência dos problemas que iremos tratar nesta tese.

Iniciamos denotando por B uma caixa $n$-dimensional, possivelmente ilimitada:

$$
B \stackrel{\text { def }}{=}\left(\left[a_{1}, b_{1}\right] \times \ldots \times\left[a_{n}, b_{n}\right]\right) \cap \mathbb{R}^{n},
$$

$-\infty \leq a_{i}<b_{i} \leq+\infty$ para $i=1, \ldots, n$.

O problema mais simples que vamos estudar é:

Definição 1.2.1. Seja $f: \mathbb{R}^{n} \rightarrow(-\infty,+\infty]$ uma função convexa, própria e fechada. 0 problema de programação convexa em caixa é o problema de

$$
\begin{array}{ll}
\min & f(x) \\
\text { s.a } & x \in B .
\end{array}
$$

Se $\mathrm{B}=\mathbb{R}^{n}$, vamos empregar também o nome problema de programação convexa irrestrito.

Estamos também interessados em problemas de otimização convexa com restrições funcionais mais gerais:

Definição 1.2.2. Sejam $f: \mathbb{R}^{n} \rightarrow(-\infty, \infty]$ e $g: \mathbb{R}^{n} \rightarrow(-\infty, \infty]^{m}$, tais que $f$ e as componentes de $g, g_{i}, i=1, \ldots, m$, são funções convexas, próprias, fechadas e para $i=1, \ldots, m$,

$$
\text { ridom } g_{i} \supset \text { ridom } f \text { e } \operatorname{dom} g_{i} \supset \operatorname{dom} f \text {. }
$$

O problema de programação convexa primal, ou simplesmente primal é o problema de

$$
\begin{array}{ll}
\min & f(x) \\
\text { s.a } & g(x) \leq 0 .
\end{array}
$$

Supomos ainda que este problema possui valor ótimo finito.

Associado ao problema primal, definimos o seu dual lagrangiano:

Definição 1.2.3. O problema dual é o problema de

$$
\begin{array}{ll}
\max & F(\lambda) \stackrel{\text { def }}{=} \inf _{x \in \mathbb{R}^{n}}\{L(x, \lambda)\} \\
\text { s.a } & \lambda \geq 0,
\end{array}
$$

no qual $L: \mathbb{R}^{n} \times \mathbb{R}^{m} \rightarrow \overline{\mathbb{R}}$ é a função lagrangiana estendida, ou simplesmente lagrangiano:

$$
L(x, \lambda) \stackrel{\text { def }}{=} f(x)+\langle\lambda, g(x)\rangle-\delta_{\mathbb{R}_{+}^{m}}(\lambda) .
$$


A ligação entre estes dois problemas tem sido objeto de estudo constante na literatura de programação não-linear e será essencial neste trabalho. Em particular, é fundamental a relação de conjugação entre a função perturbação do lado-direito, $v$, associada a (1.2) e a função objetivo dual:

Lema 1.2.4. [38, Exemplo 1 e Teorema 7] Seja $v: \mathbb{R}^{m} \rightarrow \overline{\mathbb{R}}$ dada por:

$$
\forall \lambda \in \mathbb{R}^{m}, v(\lambda) \stackrel{\text { def }}{=} \inf \left\{f(x) \mid x \in \mathbb{R}^{n}, g(x) \leq \lambda\right\}
$$

Então,

$$
\forall \lambda \in \mathbb{R}^{m}, F(\lambda)=-v^{*}(-\lambda)
$$

Outro resultado que desempenhará um papel importante nesta tese é:

Teorema 1.2.5. O conjunto de soluções duais é não vazio e limitado se, e somente se, existe $\bar{x} \in \operatorname{dom} f$ tal que $\mathrm{g}(\overline{\mathrm{x}})<0$. Chamaremos esta última condição de condição de qualificação de Slater.

Demonstração. Apesar de acreditarmos que este resultado é clássico, apresentaremos a sua demonstração uma vez que todas as referências que encontramos [21, 30, 31] assumiam que $f$ e $g_{i}, i=1, \ldots, m$, eram Lipschitz contínuas, o que não é necessariamente verdade na fronteira dos domínios efetivos de funções convexas.

1. Considere que o conjunto de soluções duais é não vazio e limitado.

Seja $\lambda^{*} \geq 0$ uma solução dual. Suponha, por contradição, que a condição de Slater não é válida:

$$
\nexists x \in \operatorname{dom} f, g(x)<0 .
$$

Usando o Teorema generalizado de Gordan [29, Teorema 4.2.3], vemos que existe $h \in \mathbb{R}_{+}^{m}$, tal que $h \neq 0$ e $\langle h, g(x)\rangle \geq 0$, para todo $x \in$ dom $f$. Então, para todo real $M>0$,

$$
\forall x \in \operatorname{dom} f, f(x)+\left\langle\lambda^{*}+M h, g(x)\right\rangle \geq f(x)+\left\langle\lambda^{*}, g(x)\right\rangle .
$$

Lembrando que $h$ é positivo e não-nulo, podemos tomar o ínfimo em $x$ e concluir que

$$
F\left(\lambda^{*}+M h\right) \geq F\left(\lambda^{*}\right) .
$$

Portanto $\lambda^{*}+M h$ também é uma solução dual. Como podemos escolher $M$ arbitrariamente grande, temos uma contradição com a limitação do conjunto de soluções duais. 
2. Considere que $\exists \bar{x} \in \operatorname{dom} f, g(\bar{x})<0$.

Usando [37, Corolário 28.2.1], vemos que o conjunto de soluções duais é não vazio. Seja $\lambda^{*} \geq 0$ uma solução dual e $F^{*}$ o valor ótimo dual, que é igual a $F\left(\lambda^{*}\right)$. Chamemos de $\mu \stackrel{\text { def }}{=} \min _{i=1, \ldots, m}\left\{\left|g_{i}(\bar{x})\right|\right\}>0$. Temos:

$$
\begin{aligned}
F^{*}+\mu\left\|\lambda^{*}\right\|_{1} & \leq F\left(\lambda^{*}\right)-\left\langle\lambda^{*}, g(\bar{x})\right\rangle \\
& =\inf _{x}\left\{f(x)+\left\langle\lambda^{*}, g(x)\right\rangle\right\}-\left\langle\lambda^{*}, g(\bar{x})\right\rangle \\
& \leq f(\bar{x}) .
\end{aligned}
$$

Portanto,

$$
\left\|\lambda^{*}\right\|_{1} \leq \frac{f(\bar{x})-F^{*}}{\mu}
$$

Uma extensão natural dos problemas de otimização acima pode ser feita usando-se operadores monótonos maximais e desigualdades variacionais. ${ }^{1}$ Neste contexto, estamos interessados em:

Definição 1.2.6. Seja $T: \mathbb{R}^{n} \rightrightarrows \mathbb{R}^{n}$ um operador monótono maximal tal que dom $T \cap$ int $\mathrm{B} \neq \emptyset .^{2} \mathrm{O}$ problema da desigualdade variacional é o problema de encontrar $x \in \mathbb{R}^{n}$ tal que:

$$
0 \in T(x)+N_{B}(x)
$$

Nesta inclusão, $N_{B}(x)$ denota o cone normal à caixa $B$ em $x$.

Caso $\mathrm{B}=\mathbb{R}^{n}$, usaremos o nome busca de zeros de $\mathrm{T}$.

\subsection{Métodos Proximais}

Considere o problema de programação convexa em caixa (1.1).

Seja $D: \mathbb{R}^{n} \times \mathbb{R}^{n} \rightarrow(-\infty, \infty]$ tal que, para cada $y \in \operatorname{int} B:$

\footnotetext{
${ }^{1} \mathrm{Na}$ verdade, o problema de busca de zeros de operadores monótonos maximais generaliza os problemas de otimização convexa, o problema de busca de zero de operadores de sela associados a funções convexo-côncavas, entre outros. Para a definição e resultados sobre operadores monótonos maximais veja [41, Capítulo 12]. Em particular, o operador subdiferencial, af, é monótono maximal [41, Teorema 12.17]; porém existem operadores monótonos maximais que não são subdiferenciais de nenhuma função [41, Teorema 12.25].

${ }^{2}$ Esta condição é necessária para garantir a maximalidade da soma de operadores: $T+N_{B}[41$, Corolário 12.44].
} 
1. $D(\cdot, y)$ é finita em uma vizinhança de $y$;

2. $\mathrm{D}(\cdot, y)$ é própria, fechada, estritamente convexa em seu domínio efetivo e atinge mínimo em $y$.

Vamos chamar de método proximal uma recursão do tipo:

$$
x^{1} \in B, x^{k+1}=\underset{x}{\operatorname{argmin}}\left\{f(x)+\alpha_{k} D\left(x, x^{k}\right)\right\},
$$

$\left\{\alpha_{k}\right\}$ é uma seqüência de reais positivos conhecidos como parâmetros de regularização. A função D receberá o nome de regularização ou distância generalizada.

Em muitas situações, é interessante considerar que a distância generalizada é separável, isto é, que existem $d_{i}: \mathbb{R} \times \mathbb{R} \rightarrow(-\infty, \infty]$ tais que

$$
D(x, y)=\sum_{i=1}^{n} d_{i}\left(x_{i}, y_{i}\right) \text {. }
$$

Além disso, podemos estar interessados em métodos proximais que permitam o uso de diferentes parâmetros de regularização para cada coordenada. Neste caso, a recursão (1.5) torna-se:

$$
x^{1} \in B, x^{k+1}=\underset{x}{\operatorname{argmin}}\left\{f(x)+\sum_{i=1}^{n} \alpha_{i}^{k} d_{i}\left(x_{i}, x_{i}^{k}\right)\right\} .
$$

Para simplificar a notação, definimos:

$$
\begin{aligned}
& \nabla_{1} \mathrm{D}(x, y) \stackrel{\text { def }}{=} \frac{\partial \mathrm{D}}{\partial x}(x, y) \\
& \mathrm{d}_{i}^{\prime}\left(x_{i}, y_{i}\right) \stackrel{\text { def }}{=} \frac{\partial d_{i}}{\partial x_{i}}\left(x_{i}, y_{i}\right) .
\end{aligned}
$$

Vamos também estudar métodos proximais para a resolução do problema de desigualdade variacional (1.4). Neste caso, (1.6) pode ser facilmente generalizada, obtendo-se:

$$
x^{1} \in \mathrm{B}, 0 \in \mathrm{T}\left(x^{\mathrm{k}+1}\right)+\operatorname{diag}\left(\alpha^{\mathrm{k}}\right) \nabla_{1} \mathrm{D}\left(x^{\mathrm{k}+1}, x^{k}\right)
$$

na qual "diag $\left(\alpha^{k}\right)$ " denota a matriz diagonal obtida a partir do vetor de parâmetros $\alpha^{\mathrm{k}}$.

Por fim, chamaremos de Método Proximal Clássico o método proximal que usa o quadrado da norma euclidiana como regularização. 
CAPÍtulo 2

\section{Métodos Proximais como Métodos de Descida e sua Relação com Métodos de Multiplicadores}

\subsection{Uma versão inexata do método proximal base- ada em propriedades de descida}

Nesta seção, vamos analisar o método de ponto proximal clássico sob a perspectiva de métodos de descida para programação não-linear. Apresentaremos também um critério para aceitar soluções aproximadas do passo proximal. Outra característica desta seção é que os resultados são obtidos de forma agradavelmente simples, apesar de já apresentarem as idéias básicas que vão permear o restante deste trabalho.

Em [40], Rockafellar provou a convergência do método proximal clássico com resolução aproximada dos sub-problemas. Para obter seus resultados, ele considerou que os erros feitos a cada iteração são somáveis. Em [43], Solodov e Svaiter obtiveram resultados mais robustos, provando que os erros relativos podem se manter constantes caso seja introduzido um passo intermediário composto por uma projeção.

A seguir, vamos apresentar uma nova prova dos resultados de [43], considerando que os operadores estão definidos no $\mathbb{R}^{n}$. Além disto, demonstraremos que é possível obter resultados de convergência no caso de otimização que não dependem do passo extra de projeção. 


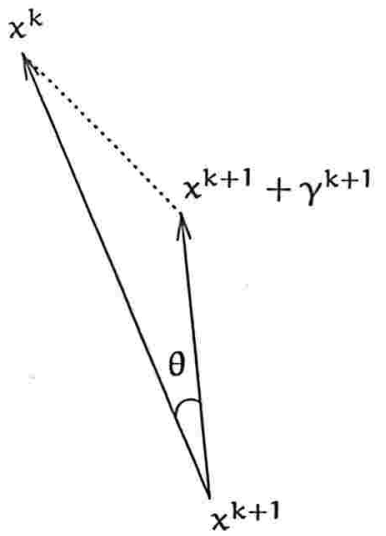

Figura 2.1: $\mathrm{O}$ ângulo $\theta$.

\section{Caracterização da descida}

Estamos interessados em estudar o problema de minimização irrestrita, introduzido na Definição 1.2.1, com a hipótese adicional que a função objetivo é finita e continuamente diferenciável em todo $\mathbb{R}^{n}$.

Uma estratégia usual para resolução deste tipo de problema é o emprego de um algoritmo que garanta o decréscimo da função objetivo, como o método de Cauchy ou estratégias baseadas em regiões de confiança. A idéia é assegurar a otimalidade dos pontos de acumulação através de um "decréscimo suficiente" ${ }^{1}$ da função objetivo.

Suponha que estamos interessados em estudar o quanto $f$ decai em uma seqüência $\left\{x^{k}\right\}$. Uma forma indireta de estimar esta queda é usar a convexidade de $f$ para concluir que:

$$
\gamma^{k+1} \stackrel{\text { def }}{=} \nabla f\left(x^{k+1}\right), \quad f\left(x^{k}\right) \geq f\left(x^{k+1}\right)+\left\langle\gamma^{k+1}, x^{k}-x^{k+1}\right\rangle .
$$

Isto é equivalente a

$$
f\left(x^{k}\right) \geq f\left(x^{k+1}\right)+\left\|\gamma^{k+1}\right\|\left\|x^{k}-x^{k+1}\right\| \cos \theta,
$$

na qual $\theta$ é o ângulo entre $\gamma^{k+1}$ e $x^{k}-\chi^{k+1}$ (como ilustra a Figura 2.1).

$\mathrm{O}$ ponto $x^{k+1}$ certamente será "melhor" do que $x^{k}$ se $\theta$ for um ângulo agudo. Considerando o triângulo $\left[x^{k}, x^{k+1}, x^{k+1}+\gamma^{k+1}\right]$, apresentado na Figura 2.1, $\theta$ será agudo se o lado oposto a ele não for o maior dos lados deste triângulo. Uma maneira de garantir esta propriedade é verificar o seguinte critério de aceitação:

$$
\left\|\gamma^{k+1}+\left(x^{k+1}-x^{k}\right)\right\| \leq \sigma \max \left\{\left\|\gamma^{k+1}\right\|,\left\|x^{k+1}-x^{k}\right\|\right\},
$$

\footnotetext{
${ }^{1}$ A palavra suficiente não é definida formalmente propositadamente. Existem várias tentativas de dar um significado preciso a suficiente. Um exemplo são os modelos básicos de algoritmos em [33].
} 
no qual $\sigma$ é um número positivo estritamente menor que 1.

Começaremos nossa análise observando algumas propriedades simples do triângulo $\left[x^{k}, x^{k+1}, x^{k+1}+\gamma^{k+1}\right]$. Chamemos de $M$ o comprimento do maior lado deste triângulo, $\left(M \stackrel{\text { def }}{=} \max \left\{\left\|\gamma^{k+1}\right\|,\left\|x^{k+1}-x^{k}\right\|\right\}\right)$ e $\alpha$ o ângulo oposto a este lado.

Lema 2.1.1. Usando a notação definida acima e (2.2),

$$
\left.0 \leq \operatorname{sen} \theta \leq \sigma \quad \text { (e portanto } \cos \theta \geq \sqrt{1-\sigma^{2}}\right)
$$

Demonstração. Usando a lei dos senos:

$$
\frac{M}{\operatorname{sen} \alpha}=\frac{\left\|\gamma^{k+1}+x^{k+1}-x^{k}\right\|}{\operatorname{sen} \theta}
$$

logo,

$$
\frac{\operatorname{sen} \theta}{\operatorname{sen} \alpha}=\frac{\left\|\gamma^{k+1}+x^{k+1}-x^{k}\right\|}{M} \leq \sigma
$$

A demonstração está concluída.

Este Lema mostra que (2.2) não só garante que $\theta$ seja um ângulo agudo, (2.2) força o ângulo $\theta$ a não se aproximar do ângulo reto, uma vez que ele deve ser menor que $\operatorname{arcsen}(\sigma)<\pi / 2$.

$\mathrm{O}$ critério de aceitação também implica que a razão entre $\left\|\gamma^{k+1}\right\|$ e $\left\|x^{k+1}-x^{k}\right\|$ é bem comportada:

Lema 2.1.2. Seja $M \stackrel{\text { def }}{=} \max \left\{\left\|\gamma^{k+1}\right\|,\left\|x^{k+1}-x^{k}\right\|\right\}$ e $m$ o menor destes dois números. Se vale (2.2), então

$$
(1-\sigma) M \leq m \leq M
$$

Demonstração. Claramente,

$$
\begin{gathered}
M-m \leq\left\|x^{k}-x^{k+1}-\gamma^{k+1}\right\| \leq \sigma M \Rightarrow \\
(1-\sigma) M \leq m .
\end{gathered}
$$


Usando os resultados acima e a estimativa do decréscimo de $f$ dada por (2.1), obtemos:

$$
\begin{aligned}
f\left(x^{k}\right) & \geq f\left(x^{k+1}\right)+(1-\sigma) \sqrt{1-\sigma^{2}} M^{2} \\
& \geq f\left(x^{k+1}\right)+(1-\sigma) \sqrt{1-\sigma^{2}}\left\|\gamma^{k+1}\right\|^{2} .
\end{aligned}
$$

Isto sugere o seguinte método de descida:

\section{Algoritmo 2.1.3. Método Proximal Inexato para Funções Diferenciáveis}

Seja $f: \mathbb{R}^{n} \rightarrow \mathbb{R}$ uma função convexa e diferenciável e seja $\sigma$ um número em $[0,1)$.

1. Inicialização: Comece $\operatorname{com} x^{1} \in \mathbb{R}^{n}$;

2. Iteração: Encontre $x^{k+1}$ tal que, para $\gamma^{k+1} \stackrel{\text { def }}{=} \nabla f\left(x^{k+1}\right)$ e $e^{k+1} \stackrel{\text { def }}{=} \gamma^{k+1}+x^{k+1}-x^{k}$,

$$
\left\|e^{k+1}\right\| \leq \sigma \max \left\{\left\|\gamma^{k+1}\right\|,\left\|x^{k+1}-x^{k}\right\|\right\} .
$$

Isto pode ser feito por meio de uma minimização aproximada de

$$
f(x)+\frac{1}{2}\left\|x-x^{k}\right\|^{2}
$$

Aplicando recursivamente a desigualdade (2.3) concluímos que

$$
f\left(x^{1}\right) \geq f\left(x^{k}\right)+(1-\sigma) \sqrt{1-\sigma^{2}} \sum_{j=2}^{k}\left\|\gamma^{j}\right\|^{2} .
$$

Portanto, a seqüência $\left\{f\left(x^{k}\right)\right\}$ é decrescente, e assim, ou ela é ilimitada inferiormente ou converge para um valor real. Neste último caso, (2.4) implica que $\gamma^{k}\left(=\nabla f\left(x^{k}\right)\right) \rightarrow 0^{2}$ o que nos leva a enunciar o seguinte resultado de convergência:

Teorema 2.1.4. Seja $f: \mathbb{R}^{n} \rightarrow \mathbb{R}$ convexa, continuamente diferenciável e limitada inferiormente. Então, todos os pontos de acumulação da seqüência gerada pelo Algoritmo 2.1.3 são soluções do problema programação de convexa irrestrito.

Demonstração. Seja $\bar{x}$ o limite de uma subseqüência $\left\{x^{k}\right\}_{k \in \mathcal{K}}$. A discussão que segue a desigualdade (2.3) mostrou que, sob as hipóteses acima, $\gamma^{k} \rightarrow 0$. Portanto, a continuidade de $\nabla f$ implica que $\nabla f(\bar{x})=0$, assim $\bar{x}$ é um mínimo de $f$.

\footnotetext{
${ }^{2} \mathrm{Na}$ verdade $\left\|\gamma^{\mathrm{k}}\right\|$ é $\mathrm{l}^{2}$, ou seja ela deve ser quadrado somável.
} 


\section{O caso não-diferenciável}

Nesta seção, vamos abrir mão das hipóteses de diferenciabilidade de f e explorar melhor sua convexidade. Isto é, vamos estudar o problema de otimização irrestrito (1.1) sem hipóteses adicionais.

O substituto natural do gradiente de f é o operador subdiferencial [41, Capítulo 8], $\partial f$, uma vez que para $\gamma^{k+1} \in \partial f\left(x^{k+1}\right)$ a desigualdade fundamental

$$
f\left(x^{k}\right) \geq f\left(x^{k+1}\right)+\left\langle\gamma^{k+1}, x^{k}-x^{k+1}\right\rangle
$$

ainda se verifica.

Seguindo a seqüência de desigualdades (2.1) a (2.3) podemos observar que, com as definições acima, elas também se mantêm válidas, em particular:

$$
f\left(x^{k}\right) \geq f\left(x^{k+1}\right)+(1-\sigma) \sqrt{1-\sigma^{2}}\left\|\gamma^{k+1}\right\|^{2} .
$$

Mais uma vez, usando esta desigualdade para motivar um algoritmo de otimização, somos levados a minimizar aproximadamente

$$
f(x)+\frac{1}{2}\left\|x-x^{k}\right\|^{2}
$$

Observe que a norma euclidiana regulariza a função objetivo original garantindo convexidade forte e, portanto, melhorando as propriedades de convergência dos algoritmos usuais de minimização. Porém, ela "obriga" o próximo iterado a se manter próximo do ponto atual e é possível que desejemos reduzir este efeito. Introduzimos então o parâmetro de regularização $\alpha_{k}$ que pode ser usado para controlar a "influência" da regularização. A função que vamos minimizar torna-se:

$$
f(x)+\frac{\alpha_{k}}{2}\left\|x-x^{k}\right\|^{2}
$$

Estas idéias materializam-se no seguinte método proximal:

\section{Algoritmo 2.1.5. Método Proximal Inexato para Funções Convexas}

Seja $f: \mathbb{R}^{n} \rightarrow(-\infty, \infty]$ uma função convexa, própria e fechada. Seja $\sigma$ um número em $[0,1)$ e $\widetilde{\alpha}$ uma constante positiva.

1. Inicialização: Comece $\operatorname{com} x^{1} \in \mathbb{R}^{n}$;

2. Iteração: Escolha $\alpha_{k}$ tal que $0<\alpha_{k} \leq \widetilde{\alpha}$. Encontre $x^{k+1}$ tal que, para $\gamma^{k+1} \in$ $\partial f\left(x^{k+1}\right)$ e $e^{k+1} \stackrel{\text { def }}{=} \gamma^{k+1}+\alpha_{k}\left(x^{k+1}-x^{k}\right)$,

$$
\left\|e^{k+1}\right\| \leq \sigma \max \left\{\left\|\gamma^{k+1}\right\|, \alpha_{k}\left\|x^{k+1}-x^{k}\right\|\right\} .
$$


Isto pode ser feito minimizando aproximadamente

$$
f(x)+\frac{\alpha_{k}}{2}\left\|x-x^{k}\right\|^{2} .
$$

Teorema 2.1.6. Seja $f: \mathbb{R}^{n} \rightarrow(-\infty, \infty]$ uma função convexa, própria, fechada $e$ limitada inferiormente. Então, todos os pontos de acumulação da seqüência gerada pelo algoritmo proximal inexato são soluções do problema convexo irrestrito.

Demonstração. Analogamente ao feito na demonstração do Teorema 2.1.4, definimos

$$
\begin{aligned}
& M \stackrel{\text { def }}{=} \max \left\{\left\|\gamma^{k+1}\right\|, \alpha_{k}\left\|x^{k+1}-x^{k}\right\|\right\} \text { e } \\
& m \stackrel{\text { def }}{=} \min \left\{\left\|\gamma^{k+1}\right\|, \alpha_{k}\left\|x^{k+1}-x^{k}\right\|\right\} .
\end{aligned}
$$

Podemos então mais uma vez percorrer o caminho que levou à desigualdade (2.3) e, com o auxílio de (2.5), mostrar que vale uma pequena variação desta desigualdade:

$$
f\left(x^{k}\right) \geq f\left(x^{k+1}\right)+\frac{1}{\alpha_{k}}(1-\sigma) \sqrt{1+\sigma^{2}}\left\|\gamma^{k+1}\right\|^{2} .
$$

Lembrando que $\left\{\alpha_{k}\right\}$ é limitada superiormente, podemos concluir mais uma vez que se $\left\{f\left(x^{k}\right)\right\}$ for limitada inferiormente, então $\gamma^{k} \rightarrow 0$. Usando a semi-continuidade exterior do operador subdiferencial concluímos que todo ponto de acumulação de $\left\{x^{k}\right\}$ minimiza f.

\section{Operadores monótonos maximais}

Métodos de ponto proximal são importantes para a solução do problema mais geral de busca de zeros de operadores monótonos maximais: dado um operador $T: \mathbb{R}^{n} \rightrightarrows \mathbb{R}^{n}$ monótono e maximal, deseja-se encontrar um ponto $x$ tal que

$$
0 \in T(x)
$$

Neste contexto ainda é possível usar as idéias geométricas que apresentamos.

Nosso ponto de partida é a versão exata do método de ponto proximal clássico, dada por:

$$
\begin{gathered}
\gamma^{k+1} \in T\left(x^{k+1}\right), \\
\gamma^{k+1}+\alpha_{k}\left(x^{k+1}-x^{k}\right)=0 .
\end{gathered}
$$


Em [40], Rockafellar introduziu alguns critérios de aproximação para relaxar a igualdade. Em [43], Solodov e Svaiter provaram que, com a introdução de um passo intermediário de projeção, é possível usar critérios de aceitação ainda mais permissivos que os de [40]. Este método é conhecido como algoritmo híbrido projeção-ponto proximal inexato. Apresentaremos uma demonstração alternativa da convergência do método proposto por Solodov e Svaiter baseada em idéias semelhantes àquelas usadas para analisar os Algoritmos 2.1.3 e 2.1.5.

$\mathrm{O}$ algoritmo híbrido projeção-ponto proximal usa um passo proximal inexato para obter um hiperplano que separa $x^{k}$ do conjunto de soluções. O novo iterado, $x^{k+1}$, será a projeção de $x^{k}$ sobre este hiperplano; logo $x^{k+1}$ estará mais próximo do conjunto de zeros de T do que $x^{k}$ (Figura 2.2). Formalmente temos:

\section{Algoritmo 2.1.7. Algoritmo Híbrido Projeção-Ponto Proximal Inexato}

Seja $T: \mathbb{R}^{n} \rightrightarrows \mathbb{R}^{n}$ um operador monótono maximal. Seja $\sigma$ um número em $[0,1)$ e $\widetilde{\alpha}$ um real positivo.

1. Inicialização: Comece $\operatorname{com} x^{1} \in \mathbb{R}^{n}$;

2. Iteração: Escolha $\alpha_{k}$ tal que $0<\alpha_{k} \leq \widetilde{\alpha}$. Encontre $\widetilde{x}^{k}$ e $\widetilde{\gamma}^{k} \in T\left(\widetilde{x}^{k}\right)$ tais que

$$
\left\|\tilde{\gamma}^{k}+\alpha_{k}\left(\tilde{x}^{k}-x^{k}\right)\right\| \leq \sigma \max \left\{\left\|\tilde{\gamma}^{k}\right\|, \alpha_{k}\left\|\tilde{x}^{k}-x^{k}\right\|\right\},
$$

o que pode ser feito através de uma resolução aproximada de

$$
0 \in T(x)+\alpha_{k}\left(x-x^{k}\right) \text {. }
$$

Então, $x^{k+1}$ é a projeção de $x^{k}$ sobre o hiperplano que passa por $\tilde{x}^{k}$ e que tem $\widetilde{\gamma}^{k}$ como normal, ou seja

$$
x^{k+1}=x^{k}-\frac{\left\langle\widetilde{\gamma}^{k}, x^{k}-\widetilde{x}^{k}\right\rangle}{\left\|\widetilde{\gamma}^{k}\right\|^{2}} \widetilde{\gamma}^{k} \text {. }
$$

Destacamos que o algoritmo descrito acima é análogo ao Algoritmo 2.1.5, porém o caso de otimização não necessita da projeção. A necessidade da projeção deve-se à ausência de uma função de mérito explícita para o caso geral de operadores monótonos: não existe uma função sendo minimizada. A projeção garante que o novo ponto $x^{k+1}$, estará mais próximo dos zeros de $T$ do que $x^{k}$. Isto é uma conseqüência direta da monotonicidade de $T$, pois para todo $x^{*} \in T^{-1}(0)$,

$$
\left\langle x^{*}-\tilde{x}^{k}, \tilde{\gamma}^{k}-0^{k}\right\rangle=\left\langle x^{*}-\tilde{x}^{k}, \tilde{\gamma}^{k}\right\rangle \leq 0,
$$

e, como já vimos, o ângulo $\theta$ entre $x^{k}-\widetilde{x}^{k}$ e $\widetilde{\gamma}^{k}$ é agudo, o que garante que

$$
\left\langle x^{k}-\tilde{x}^{k}, \tilde{\gamma}^{k}\right\rangle>0 \text {. }
$$




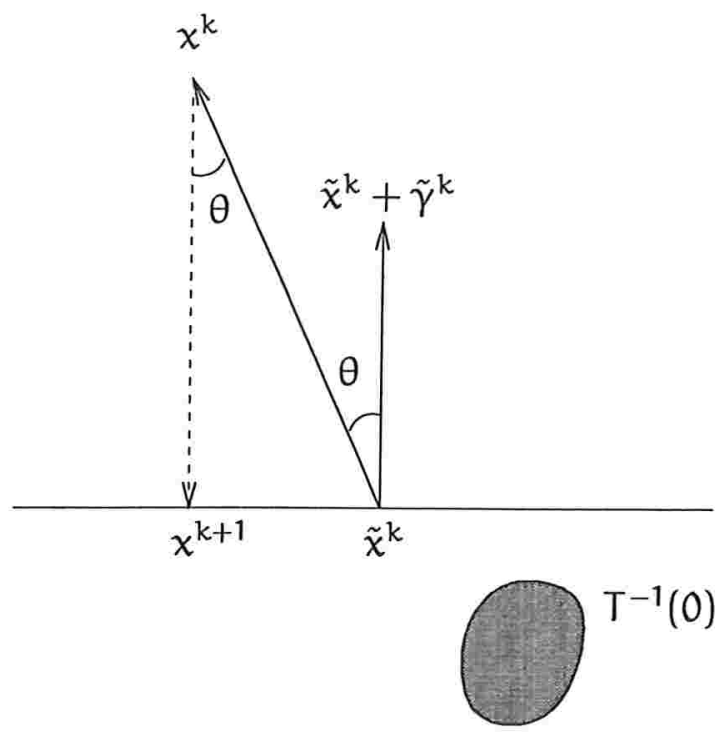

Figura 2.2: A projeção após o passo proximal aproximado.

Desta forma o hiperplano que passa por $\widetilde{\chi}^{k}$ e tem $\widetilde{\gamma}^{k}$ como normal separa estritamente $x^{k}$ e $T^{-1}(0)$ (como sugere a Figura 2.2). Já que $x^{k+1}$ é a projeção de $x^{k}$ sobre este hiperplano, temos que para todo $x^{*} \in T^{-1}(0)$ :

$$
\left\|x^{k+1}-x^{*}\right\|^{2} \leq\left\|x^{k}-x^{*}\right\|^{2}-\left\|x^{k+1}-x^{k}\right\|^{2} .
$$

Assim, a função que decai a cada iteração é a distância ao conjunto solução. Podemos enunciar o principal resultado desta seção:

Teorema 2.1.8. Seja $\mathrm{T}: \mathbb{R}^{n} \rightrightarrows \mathbb{R}^{n}$ monótono e maximal. Suponha ainda que T possui pelo menos um zero. Então, a seqüência gerada pelo algoritmo híbrido projeção-ponto proximal inexato converge para um zero de $\mathrm{T}$.

Demonstração. Mais uma vez, chamamos de $\theta$ o ângulo entre $\chi^{k}-\widetilde{\chi}^{k}$ e $\widetilde{\gamma}^{k}$ e definimos

$$
\begin{aligned}
& M \stackrel{\text { def }}{=} \max \left\{\left\|\tilde{\gamma}^{k}\right\|, \alpha_{k}\left\|\tilde{x}^{k}-x^{k}\right\|\right\} \text { e } \\
& m \stackrel{\text { def }}{=} \min \left\{\left\|\tilde{\gamma}^{k}\right\|, \alpha_{k}\left\|\tilde{x}^{k}-x^{k}\right\|\right\} .
\end{aligned}
$$

Usando os Lemas 2.1.1 e 2.1.2, concluímos que

$$
\begin{gathered}
\cos \theta \geq \sqrt{1-\sigma^{2}}, \\
\left\|\widetilde{\gamma}^{k}\right\| \leq \frac{\alpha_{k}\left\|\widetilde{x}^{k}-\chi^{k}\right\|}{1-\sigma} .
\end{gathered}
$$


Seja $x^{*}$ um zero de T. Usando a equação (2.8), concluímos que $\left\|x^{k}-x^{*}\right\|$ é uma seqüência não-crescente e limitada inferiormente (por 0 ) e que $\left\|x^{k+1}-x^{k}\right\| \rightarrow 0$. Já a definição de $x^{k+1} \operatorname{diz}$ que

$$
\left\|x^{k+1}-x^{k}\right\|=\left\|\tilde{x}^{k}-x^{k}\right\| \cos \theta
$$

o que, junto a (2.9), leva-nos a concluir que

$$
\left\|\tilde{x}^{k}-x^{k}\right\| \rightarrow 0
$$

Como $\left\{\alpha_{k}\right\}$ é limitada superiormente, (2.10) implica que

$$
\tilde{\gamma}^{k} \rightarrow 0
$$

Por outro lado, sabemos que $\left\|x^{k}-x^{*}\right\|$ é não-crescente, logo $\left\{x^{k}\right\}$ é limitada, pois está contida na bola de centro $x^{1}$ e raio $\left\|x^{1}-x^{*}\right\|$. Seja $\bar{x}$ ponto de acumulação de $\left\{x^{k}\right\}$. A Equação (2.11) diz que $\bar{x}$ também é ponto de acumulação de $\left\{\widetilde{x}^{k}\right\}$. Lembrando de (2.12), a semi-continuidade exterior de $T$ implica então que $0 \in \mathrm{T}(\bar{x})$.

Por fim, aplicando a desigualdade (2.8) para $x^{*}=\bar{x}$ concluímos que $\left\|x^{k}-\bar{x}\right\|$ é nãocrescente e que $\left\|x^{k}-\bar{x}\right\|$ converge para zero em alguma subseqüencia. Logo, $\left\|x^{k}-\bar{x}\right\| \rightarrow 0$, ou seja, $x^{k} \rightarrow \bar{x}$.

\subsection{Conexão com métodos de multiplicadores}

Em [39], Rockafellar mostrou que o método de multiplicadores clássico, introduzido por Hestenes [22] e Powell [34], pode ser interpretado como uma forma indireta de aplicar o método proximal clássico à solução do dual de um problema de programação convexa, no qual cada passo proximal equivale a uma minimização irrestrita envolvendo apenas os dados primais.

Essa técnica foi amplamente explorada na literatura para a obtenção de diferentes métodos de multiplicadores a partir de variações do método proximal clássico, como em $[8,28,27,6,5,3,25,42]$.

Esta seção dedica-se a apresentar, independentemente do termo regularizador usado no método proximal, a relação entre métodos de multiplicadores e métodos proximais. A nossa abordagem baseia-se na análise ligando os métodos clássicos contida em [7, seções 5.4.5, 5.4.6] e estendida para regularizações gerais em [25, 42].

Durante o restante desta seção vamos estudar o problema de programação convexa descrito na Definição 1.2.2. Lembramos que consideramos que o valor ótimo deste problema é finito. A ferramenta básica que nós vamos explorar é a relação entre o primal 
e seu dual lagrangiano, sobretudo o Lema 1.2.4, bem como resultados de dualidade de Fenchel [36] e [37, Capítulo 31].

Estamos interessados em relacionar soluções da maximização da função objetivo dual perturbada por um termo côncavo (como é feito em métodos proximais) com soluções da minimização de penalizações do problema primal.

Definição 2.2.1. Uma penalidade é uma função $P: \mathbb{R}^{m} \rightarrow(-\infty, \infty]$ convexa, própria, fechada, não-decrescente $(u \geq v \Rightarrow P(u) \geq P(v))$ e tal que $0 \in \operatorname{dom} P{ }^{3}$

Definimos ainda a seguinte notação: se $x \in \mathbb{R}^{n}, P(g(x))$ denota a composição de $P$ e $g$, caso $g(x) \in \mathbb{R}^{m}$, ou $+\infty$, caso contrário.

Chamaremos de penalização do problema primal ou de problema primal penalizado um problema na forma

$$
\begin{array}{ll}
\min & f(x)+P(g(x)) \\
\text { s.a } & x \in \mathbb{R}^{n} .
\end{array}
$$

Uma caracterização alternativa da monotonicidade das penalidades ${ }^{4}$ é dada no lema a seguir:

Lema 2.2.2. Seja $h: \mathbb{R}^{m} \rightarrow(-\infty, \infty]$ uma função convexa própria e fechada. Então $\mathrm{h}$ é não-decrescente se, e somente se, dom $\mathrm{h}^{*} \subset \mathbb{R}_{+}^{\mathrm{m}}$.

Demonstração.

- $h$ não-decrescente $\Rightarrow \operatorname{dom} h^{*} \subset \mathbb{R}_{+}^{m}$.

Seja $y \in \mathbb{R}^{m}$ tal que $y_{j}<0$, para algum $j$. Seja $x \in \operatorname{dom} h$ e denotemos por $e^{j}$ o j-ésimo vetor da base canônica. Temos

$$
\begin{aligned}
\forall \alpha>0, h^{*}(y) & \geq\left\langle x-\alpha e^{j}, y\right\rangle-h\left(x-\alpha e^{j}\right) \\
& \geq\langle x, y\rangle+\alpha\left|y_{j}\right|-h(x) \Rightarrow \\
h^{*}(y) & =\infty .
\end{aligned}
$$

- $\operatorname{dom} h^{*} \subset \mathbb{R}_{+}^{m} \Rightarrow h$ não-decrescente.

Como h é fechada,

$$
\begin{aligned}
h(x)=h^{* *}(x) & =\sup _{y}\left\{\langle y, x\rangle-h^{*}(y)\right\} \\
& =\sup _{y \geq 0}\left\{\langle y, x\rangle-h^{*}(y)\right\} .
\end{aligned}
$$

\footnotetext{
${ }^{3} 0 \in \operatorname{dom} P$ e $P$ não-decrescente, implicam que $\operatorname{dom} P \supset \mathbb{R}_{-}^{m}$.

${ }^{4} \mathrm{O}$ fato de serem não-decrescentes.
} 
Além disto, dados $a \geq b$ e $y \geq 0$ :

$$
\begin{aligned}
\langle y, a\rangle & \geq\langle y, b\rangle \Rightarrow \\
\langle y, a\rangle-h^{*}(y) & \geq\langle y, b\rangle-h^{*}(y) \stackrel{(2.13)}{\Rightarrow} \\
h(a) & \geq h(b) .
\end{aligned}
$$

Outro resultado fundamental é:

Lema 2.2.3. Seja $\mathrm{P}: \mathbb{R}^{\mathrm{m}} \rightarrow(-\infty, \infty]$ uma penalidade. Seja $\mathrm{g}: \mathbb{R}^{\mathrm{n}} \rightarrow(-\infty, \infty]^{\mathrm{m}}$ a função restrição do problema primal. Então, a função $\mathrm{P}(\mathrm{g}(\cdot)): \mathbb{R}^{n} \rightarrow(-\infty, \infty]$, definida em 2.2.1, é própria e convexa.

Demonstração. Para mostrar que $\mathrm{P}(g(\cdot))$ é própria, basta provar que ela é finita em algum ponto $\bar{x} \in \mathbb{R}^{n}$. Seja $\bar{x}$ um ponto viável primal. Então $P(g(\bar{x})) \leq P(0) \in \mathbb{R}$.

Agora, seja $\zeta \in[0,1]$ e $x$ e $y$ dois pontos em que $P(g(\cdot))$ é finita. Temos:

$$
\begin{aligned}
\mathrm{P}(\mathrm{g}(\zeta x+(1-\zeta) y)) & \leq \mathrm{P}(\zeta \mathrm{g}(\mathrm{x})+(1-\zeta) \mathrm{g}(\mathrm{y})) & & \text { [Conv. g e } \mathrm{P} \text { não-dec.] } \\
& \leq \zeta \mathrm{P}(\mathrm{g}(\mathrm{x}))+(1-\zeta) \mathrm{P}(\mathrm{g}(\mathrm{y})) . & & {[\text { Conv. de } \mathrm{P}] }
\end{aligned}
$$

Lema 2.2.4. Sejam $\mathrm{P}, \mathrm{g}$ e $\mathrm{P}(\mathrm{g}(\cdot))$ como no lema acima. Se dom $\mathrm{P}^{*} \cap \mathbb{R}_{++}^{\mathrm{m}} \neq \emptyset$, então $\mathrm{P}(\mathrm{g}(\cdot))$ é fechada.

Demonstração. Sabemos que $\mathrm{P}=\mathrm{P}^{* *}$. Isto nos leva a tentar provar que:

$$
\forall x \in \mathbb{R}^{n}, P(g(x))=\sup _{v \in \operatorname{dom} P^{*}}\left\{\langle v, g(x)\rangle-P^{*}(v)\right\} .
$$

Neste caso, como o Lema 2.2.2 garante que dom $\mathrm{P}^{*} \subset \mathbb{R}_{+}^{m}, \mathrm{P}(\mathrm{g}(\cdot))$ será fechada, por ser o supremo pontual de funções fechadas [41, Proposição 1.26].

A definição de $P$ assegura que a equação acima vale sempre que $g(x) \in \mathbb{R}^{m}$. Portanto, basta provarmos que, se $g_{i}(x)=+\infty$, para algum $i=1, \ldots, m$, então o supremo acima também vale $+\infty$. Seja $v \in \operatorname{dom} P^{*} \cap \mathbb{R}_{++}^{m}$. Temos:

$$
\langle v, g(x)\rangle-P^{*}(v)=v_{i}(+\infty)+\sum_{\substack{j=1, \ldots, m \\ j \neq i}} v_{j} g_{j}(x)-P^{*}(v)=+\infty .
$$


No lema anterior assumimos a hipótese que dom $P^{*} \cap \mathbb{R}_{++}^{m} \neq \emptyset$, que será natural em nossas aplicações, uma vez que sempre conheceremos $\mathrm{P}^{*}$. Porém, esta condição pode ser substituída pela restrição equivalente que $\mathrm{P}$ depende de todas suas variáveis, isto é,

$\nexists i \in\{1, \ldots, m\}$ tal que $\forall u \in \mathbb{R}^{m}$,

$$
P\left(u_{1}, \ldots, u_{i-1}, u_{i}, u_{i+1}, \ldots, u_{m}\right)=P\left(u_{1}, \ldots, u_{i-1}, 0, u_{i+1}, \ldots, u_{m}\right) .
$$

Esta hipótese é mais adequada se não conhecermos $\mathrm{P}^{*}$. Esta última condição é natural pois, caso $P$ não dependa de alguma de suas variáveis, o respectivo problema penalizado iria "esquecer" uma restrição.

Notamos ainda que não basta que $P$ seja uma penalidade para que $P(g(\cdot))$ seja fechada. Um exemplo disso é $P\left(u_{1}, u_{2}\right)=\max \left(u_{2},-1\right)$ (neste caso, $P *\left(u_{1}, u 2\right)=+\infty$, se $\left.u_{1} \neq 0\right)$. Para $g_{1}(x)=1 / x-1+\delta_{\mathbb{R}_{++}}(x)$ e $g_{2}(x)=x-1$, temos

$$
P\left(g_{1}(x), g_{2}(x)\right)= \begin{cases}+\infty & \text { se } x \leq 0 \\ x-1 & \text { se } x>0\end{cases}
$$

que não é fechada.

Apoiados nessas definições e resultados, podemos enfocar a relação entre problemas penalizados e problemas duais perturbados. Um primeiro, e importante, passo nesta direção foi feito em [7, seção 5.4.5]). Apresentamos a seguir uma pequena extensão deste resultado:

Proposição 2.2.5. ([7, seção 5.4.5]) Seja $\mathrm{P}$ uma penalidade, $v$ a função perturbação do lado-direito e F a função objetivo dual. Se $\operatorname{ridom} v \cap \operatorname{ridom} P \neq \emptyset$ ou $\operatorname{ridom} \mathrm{F} \cap$ ridom $P^{*} \neq \emptyset$, então:

$$
\inf _{x \in \mathbb{R}^{n}}\{f(x)+P(g(x))\}=\inf _{u \in \mathbb{R}^{m}}\{v(u)+P(u)\}=\sup _{\lambda \in \mathbb{R}_{+}^{m}}\left\{F(\lambda)-P^{*}(\lambda)\right\} .
$$

Demonstração. Como P é não-decrescente:

$$
\begin{aligned}
\inf _{x \in \mathbb{R}^{n}}\{f(x)+P(g(x))\} & =\inf _{x \in \mathbb{R}^{n}, g(x) \in \mathbb{R}^{m}}\{f(x)+P(g(x))\} \\
& =\inf _{u \in \mathbb{R}^{m}} \inf _{x \in \mathbb{R}^{n}, g(x) \leq u}\{f(x)+P(g(x))\} \\
& \leq \inf _{u \in \mathbb{R}^{m}} \inf _{x \in \mathbb{R}^{n}, g(x) \leq u}\{f(x)+P(u)\} \\
& =\inf _{u \in \mathbb{R}^{m}}\{v(u)+P(u)\} .
\end{aligned}
$$

Por outro lado,

$$
\begin{aligned}
\inf _{x \in \mathbb{R}^{n}}\{f(x)+P(g(x))\} & \geq \inf _{x \in \mathbb{R}^{n}, g(x) \in \mathbb{R}^{m}}\{v(g(x))+P(g(x))\} \\
& \geq \inf _{u \in \mathbb{R}^{m}}\{v(u)+P(u)\} .
\end{aligned}
$$


Portando, a primeira igualdade está provada.

Já que assumimos que o valor ótimo primal é real, $F$ e $v$ serão funções próprias. Podemos então usar o Lema 1.2.4 e o Teorema de dualidade de Fenchel [37, Teorema 31.1], para concluir que:

$$
\inf _{u \in \mathbb{R}^{m}}\{v(u)+P(u)\}=\sup _{\lambda \in \mathbb{R}^{m}}\left\{F(\lambda)-P^{*}(\lambda)\right\} .
$$

Como fora do ortante positivo $F$ vale $-\infty$ e $P$ vale $+\infty$, a segunda igualdade está demonstrada.

Infelizmente este resultado relaciona apenas os valores ótimos dos problemas e não suas soluções, como desejamos. Contudo, se a perturbação do dual for estritamente convexa, é possível recuperar a solução da otimização dual a partir de uma solução qualquer do problema primal penalizado, como mostramos a seguir:

Teorema 2.2.6. Seja D: $\mathbb{R}^{m} \rightarrow(-\infty, \infty]$ uma função estritamente convexa, própria, fechada e que atinge mínimo no $\mathbb{R}_{+}^{m}$. Se $\mathrm{P}$ é a função convexo conjugada de $\mathrm{D}+\delta_{\mathbb{R}_{+}^{m}}$, então $\mathrm{P}$ é uma penalidade. Além disto, se

$$
\begin{aligned}
& \bar{\lambda}=\underset{\lambda \in \mathbb{R}_{+}^{m}}{\operatorname{argmax}}\{F(\lambda)-D(\lambda)\} e \\
& \bar{x} \in \underset{x \in \mathbb{R}^{n}}{\operatorname{Argmin}}\{f(x)+P(g(x))\},
\end{aligned}
$$

então

$$
\bar{\lambda}=\nabla P(g(\bar{x}))
$$

Demonstração. Vamos mostrar que $\mathrm{P}$ é uma penalidade. Claramente $\mathrm{P}$ é convexa, própria e fechada, pois é a função conjugada de uma função desta classe. O Lema 2.2.2 garante que $P$ é não-decrescente. Além do mais, se $\bar{u} \in \mathbb{R}_{+}^{m}$ é um ponto de mínimo de $\mathrm{D}$, então

$$
0 \in \partial\left(D+\delta_{\mathbb{R}_{+}^{m}}\right)(\bar{u}) \Leftrightarrow \bar{u} \in \partial P(0) .
$$

logo $\mathrm{P}$ é finita no 0. Portanto, $\mathrm{P}$ é uma penalidade.

Para a demonstrar a segunda parte do teorema, faremos uso de:

Teorema 2.2.7. [36, Teorema 2] Seja $\mathrm{f}$ (g) uma função convexo (côncava) e própria no $\mathbb{R}^{n}$. Suponha que a desigualdade de Fenchel seja válida como uma igualdade. Então, $\bar{\chi}$ é um ponto onde $\mathrm{f}-\mathrm{g}$ atinge mínimo, se, e somente se, $\partial \mathrm{f}(\overline{\mathrm{x}})$ e $\partial \mathrm{g}(\overline{\mathrm{x}})$ tiverem um ponto em comum. Além disto, a intersecção $\partial \mathrm{f}(\overline{\mathrm{x}}) \cap \partial \mathrm{g}(\overline{\mathrm{x}})$ coincide com o conjunto dos pontos onde $\mathrm{g}_{*}-\mathrm{f}^{*}$ atinge máximo. 
Lembrando de (2.15), vemos que $P$ é subdiferenciável na origem. Como $P^{*}(=D+$ $\delta_{\mathbb{R}_{+}^{\mathfrak{m}}}$ ) é estritamente convexa, concluímos que $0 \in \operatorname{int} \operatorname{dom} P$ [41, Teorema 11.13]. Por outro lado, assumimos que o valor ótimo do problema primal é finito, e deste modo $0 \in \operatorname{dom} v$. Logo, 0 é aderente a ri dom $v$. Concluímos que int $\operatorname{dom} P \cap$ ri dom $v \neq \emptyset$.

Usando a Proposição 2.2.5 obtemos:

$$
\begin{aligned}
\inf _{x \in \mathbb{R}^{n}}\{f(x)+P(g(x))\} & =\inf _{u \in \mathbb{R}^{m}}\{v(u)+P(u)\} \\
& =\sup _{\lambda \in \mathbb{R}_{+}^{m}}\{F(\lambda)-D(\lambda)\} .
\end{aligned}
$$

A definição de $\bar{x}$ e o fato de $P$ ser não-decrescente garantem que $f(\bar{x})=v(g(\bar{x}))$. Portando, definido-se $\bar{u} \stackrel{\text { def }}{=} \mathrm{g}(\bar{x})$, temos que

$$
\begin{aligned}
v(\bar{u})+P(\bar{u}) & =\inf _{u \in \mathbb{R}^{m}}\{v(u)+P(u)\} \\
& =\sup _{\lambda \in \mathbb{R}_{+}^{m}}\{F(\lambda)-D(\lambda)\} .
\end{aligned}
$$

O Lema 1.2.4 mostra que podemos usar o Teorema 2.2.7 para concluir que:

$$
\bar{\lambda}=\underset{\lambda \in \mathbb{R}^{m}}{\operatorname{argmax}}\{F(\lambda)-D(\lambda)\} \in \partial P(\bar{u})=\{\nabla P(g(\bar{x}))\} .
$$

A última igualdade é uma conseqüência do fato de $P$ ser a função convexo conjugada de uma função estritamente convexa, logo $\mathrm{P}$ é diferenciável sempre que for subdiferenciável [41, Teorema 11.13].

Para aplicar este resultado à resolução do problema dual por um método proximal, basta lembrarmos que dada uma distância generalizada $D, D\left(\cdot, \lambda^{k}\right)$ é estritamente convexa, própria, fechada e atinge mínimo em $\lambda^{k}$. Logo o Teorema 2.2 .6 diz que o passo proximal,

$$
\lambda^{k+1}=\underset{\lambda \in \mathbb{R}_{+}^{m}}{\operatorname{argmax}}\left\{F(\lambda)-D\left(\lambda, \lambda^{k}\right)\right\},
$$

pode ser feito minimizando um problema primal penalizado por

$$
P=\left(D\left(\cdot, \lambda^{k}\right)+\delta_{\mathbb{R}_{+}^{m}}(\cdot)\right)^{*},
$$

sempre que este problema tiver soluções.

Desejamos assim encontrar condições que garantam que o problema primal penalizado tenha soluções, assegurando que o método de multiplicadores proposto esteja bem definido. Apresentamos uma resposta parcial a esta pergunta.

Mais uma vez, chamaremos a função convexo conjugada de $\left(D+\delta_{\mathbb{R}_{+}^{m}}\right)$ de $P$. Para facilitar a apresentação usaremos também a seguinte convenção:

$$
\forall x \in \mathbb{R}^{n}, p(x) \stackrel{\text { def }}{=} \mathrm{P}(g(x)) .
$$


Lema 2.2.8. Seja D : $\mathbb{R}^{\mathrm{m}} \rightarrow(-\infty, \infty]$ convexa, própria e fechada atingindo mínimo no $\mathbb{R}_{+}^{\mathrm{m}}$. Suponha ainda que dom D $\supset \mathbb{R}_{++}^{\mathrm{m}}$. Seja $\mathcal{R} \subset \mathbb{R}^{\mathrm{n}}$ a intersecção dos cones de recessão dos conjuntos de nível das componentes da função de restrição, g. Então,

$$
p^{\infty}(\mathrm{d})= \begin{cases}0 & \text { se } \mathrm{d} \in \mathcal{R} ; \\ +\infty & \text { se } \mathrm{d} \notin \mathcal{R} .\end{cases}
$$

Demonstração. Usando o Lema 2.2.4, sabemos que $p$ é uma função convexa, própria e fechada. Seja $\bar{x}$ um ponto viável primal, como dom $P \supset \mathbb{R}_{-}^{m}$, temos que $\bar{x} \in \operatorname{dom} p$, logo:

$$
p^{\infty}(d)=\lim _{t \rightarrow \infty} \frac{p(\bar{x}+t d)-p(\bar{x})}{t}
$$

para qualquer $d \in \mathbb{R}^{m}$.

Por outro lado, como int dom $\left(\mathrm{D}+\delta_{\mathbb{R}_{+}^{m}}\right)=\mathbb{R}_{++}$, sabemos que

$$
\begin{gathered}
\forall \lambda \in \mathbb{R}_{++}^{m}, \exists \gamma, \gamma \in \partial\left(\mathrm{D}+\delta_{\mathbb{R}_{+}^{m}}\right)(\lambda) \Leftrightarrow \\
\forall \lambda \in \mathbb{R}_{++}^{m}, \exists \gamma, \lambda \in \partial P(\gamma) .
\end{gathered}
$$

Portando, para todo $t>0$ e todo $\lambda \in \mathbb{R}_{++}^{m}$ :

$$
\exists \gamma, P(\gamma)+\langle\lambda, g(\bar{x}+t d)-\gamma\rangle-p(\bar{x}) \leq p(\bar{x}+t d)-p(\bar{x}) .
$$

Dividindo por $t$ e tomando o limite para $t \rightarrow \infty$, segue de (2.16) que para todo $\lambda \in \mathbb{R}_{++}^{m}$ :

$$
\left\langle\lambda, g^{\infty}(d)\right\rangle \leq p^{\infty}(d),
$$

já que a função de recessão de uma função convexa própria é uma função própria. Tomando o limite para $\lambda \rightarrow 0$ concluímos que:

$$
\forall \mathrm{d} \in \mathbb{R}^{\mathrm{m}}, 0 \leq \mathrm{p}^{\infty}(\mathrm{d})
$$

Agora, basta considerar:

1. $\mathrm{d} \in \mathcal{R}$.

$$
\begin{gathered}
g(\bar{x}+t d) \leq g(\bar{x}), \forall t \geq 0 \Rightarrow \\
p(\bar{x}+t d)-p(\bar{x}) \leq 0, \forall t \geq 0 .
\end{gathered}
$$

Dividindo por $t$ e tomando o limite para $t \rightarrow \infty$, segue que

$$
p^{\infty}(d) \leq 0 \text {. }
$$

Usando (2.18), concluímos que:

$$
p^{\infty}(d)=0
$$


2. $\mathrm{d} \notin \mathcal{R}$.

Sem perda de generalidade, vamos supor que d não está no cone de recessão de $g_{1}(\cdot)$ :

$$
g_{1}^{\infty}(d)>\zeta>0
$$

Seja $\lambda=(M, 1,1, \ldots, 1)^{\mathrm{t}} \in \mathbb{R}^{\mathrm{m}}$. Por $(2.17)$, concluímos que

$$
\forall M>0, M \zeta+\sum_{i=2}^{m} g_{i}^{\infty}(d) \leq p^{\infty}(d) .
$$

Como $g_{i}^{\infty}(d)>-\infty, i=1, \ldots, m$, basta tomar o limite para $M \rightarrow \infty$ para concluir que:

$$
p^{\infty}(d)=+\infty
$$

Lema 2.2.9. Seja $\mathrm{D}: \mathbb{R}^{m} \rightarrow(-\infty, \infty]$ convexa, própria e fechada e atingindo mínimo no $\mathbb{R}_{+}^{\mathrm{m}}$. Suponha ainda que dom D $\supset \mathbb{R}_{++}^{\mathrm{m}}$. Entâo $\mathrm{f}(\cdot)+\mathrm{P}(\mathrm{g}(\cdot))$ é convexa, própria $e$ fechada. Se o conjunto de soluções de $(P)$ for compacto e não-vazio, então os conjuntos de nivel de $\mathrm{f}(\cdot)+\mathrm{P}(\mathrm{g}(\cdot))$ são compactos. Isto garante a existência de mínimos desta função.

Demonstração. O Lema 2.2 .4 garante que $f(\cdot)+P(g(\cdot))$ é convexa e fechada. Além disto, como o primal é viável e dom $P \supset \mathbb{R}_{-}^{m}$, ela é própria.

Usando o lema anterior e [41, Exercício 3.29] concluímos que:

$$
\left(f(\cdot)+P(g(\cdot))^{\infty}(d)= \begin{cases}f^{\infty}(d) & \text { se } d \in \mathcal{R} ; \\ +\infty & \text { se } d \notin \mathcal{R} .\end{cases}\right.
$$

$\mathrm{O}$ resultado é então conseqüência da compacidade do conjunto de soluções de $(\mathrm{P})$, o que implica que $f^{\infty}(d)>0$, se $d \in \mathcal{R}[8$, Seção 5.3].

Destacamos que a hipótese dom $D \supset \mathbb{R}_{++}^{m}$ é bastante comum na literatura. Por exemplo, ela aparece em [8, p. 306], na descrição da classe de penalidades $P_{I}$ e, mais recentemente, em [1], correspondendo às propriedades de recessão exigidas das penalidades $\theta$ e à escolha do parâmetro de penalização.

Além disto, esta condição não pode ser eliminada do enunciado do Lema 2.2.9. Para justificar esta afirmação, considere $D(\lambda)=-\sqrt{1-(2-\lambda)^{2}}$, cujo domínio é $[1,3]$. Neste caso, a penalidade torna-se $P(u)=\left(D+\delta_{\mathbb{R}_{+}^{m}}\right)^{*}(u)=2 u+\sqrt{u^{2}+1}$, representada na Figura 2.2. Como $P$ tem inclinação mínima positiva: se $u \rightarrow-\infty, P$ converge para $-\infty$ 


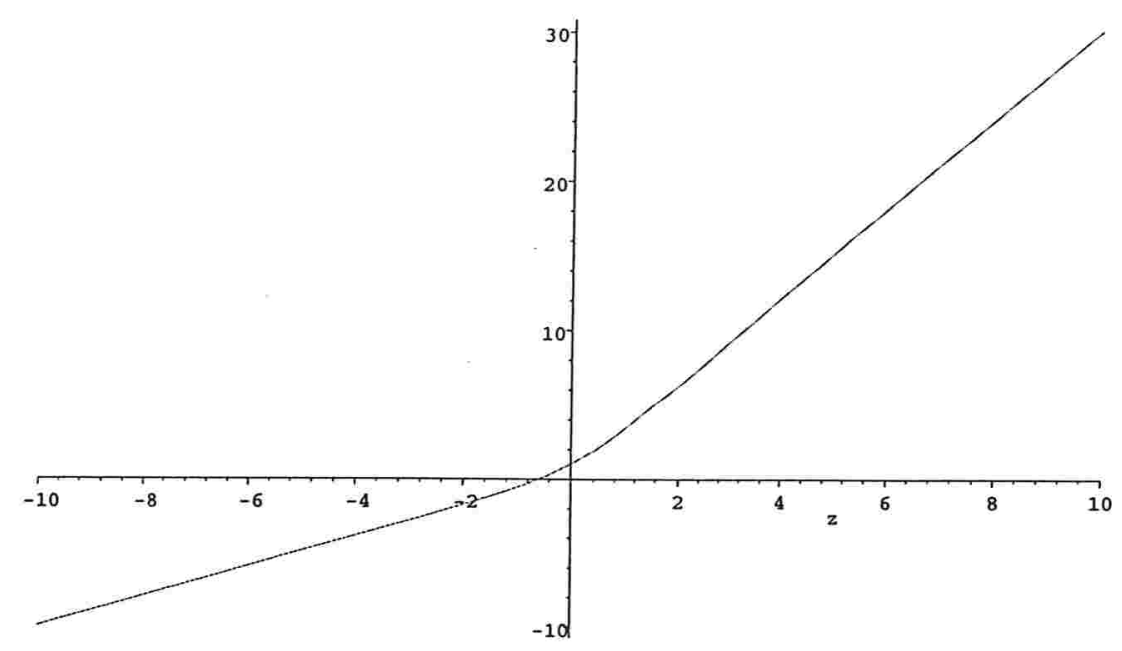

Figura 2.3: Penalidade $P(u)=2 u+\sqrt{u^{2}+1}$, associada a $D(\lambda)=-\sqrt{1-(2-\lambda)^{2}}$. Note que a penalidade decresce linearmente quando $u$ converge para $-\infty$.

pelo menos linearmente. Logo, é fácil criar um problema de programação linear que possui conjunto de solução compacto e tal que o problema penalizado por $\mathrm{P}$ não possua soluções:

$$
\begin{array}{ll}
\min & x \\
\text { s.a } & -2 x \leq 0
\end{array}
$$

O problema penalizado é:

$$
f(x)+P(g(x))=-3 x+\sqrt{4 x^{2}+1} .
$$

Esta função não possui conjuntos de nível limitados, na verdade ela converge para $-\infty$ se $x \rightarrow+\infty$. Neste caso, a penalidade faz com que a viabilidade seja mais importante que a otimalidade. Este comportamento seria revertido se a função objetivo fosse capaz de "compensar a inclinação negativa" introduzida pela penalidade, por exemplo, se ela fosse $5 x$ ao invés de $x$. 


\section{Distâncias Baseadas em Translação}

Neste capítulo, apresentamos uma classe de métodos proximais para otimização, denominada aqui de $\phi$-MPP, baseada em translações de funções estritamente convexas. $\mathrm{Na}$ Seção 3.1, estudamos a convergência supondo que os sub-problemas são resolvidos exatamente. Esta hipótese é relaxada a seguir, na Seção 3.2, na qual introduzimos um critério de aceitação que permite erros relativos fixos, semelhante ao que foi feito na Seção 2.1. A Seção 3.3 dedica-se à análise dos métodos de multiplicadores baseados no $\phi$-MPP. Este capítulo baseia-se em [25].

\subsection{Métodos de ponto proximal e funções estrita- mente convexas}

Vamos estudar o problema de minimização irrestrita, apresentado na Definição 1.2.1. Assumimos também que:

Hipótese 3.1.1. A função objetivo, $f$, é limitada inferiormente no $\mathbb{R}^{n}$.

Com este objetivo, vamos introduzir um método proximal baseado em regularizações, denotadas por $\phi$, tais que:

Hipótese 3.1.2. $\phi: \mathbb{R}^{n} \rightarrow(-\infty, \infty]$ é uma função estritamente convexa e fechada, $\phi(0)=0$ e $\phi$ é diferenciável na origem com $\nabla \phi(0)=0 .^{1}$

Uma conseqüência imediata é que $\phi$ possui conjuntos de nível compactos. Eles são fechados porque $\phi$ é fechada e são limitados porque $\phi$ possui um único mínimo [41, Proposição 3.23].

\footnotetext{
${ }^{1}$ A hipótese de $\phi(0)=0$ não é necessária para obtenção dos nosso resultados, porém ela simplifica praticamente todas as equações. Em particular, ela implica que $\phi$ é não-negativa.
} 
Apresentamos nosso método proximal:

Algoritmo 3.1.3. Método de Ponto Proximal Baseado em Translações de Funções Estritamente Convexas ( $\phi-M P P$ )

Considere o problema de minimização irrestrita, definido em 1.2.1. Suponha que as Hipótese 3.1.1 e 3.1.2 são validas.

Seja $\widetilde{\alpha}$ um número real positivo.

1. Inicialização: Comece com $x^{1} \in \operatorname{dom} f$.

2. Iteração: Escolha $0<\alpha_{k} \leq \widetilde{\alpha}$ e calcule

$$
x^{k+1} \stackrel{\text { def }}{=} \underset{x \in \mathbb{R}^{n}}{\operatorname{argmin}}\left\{f(x)+\alpha_{k} \phi\left(x-x^{k}\right)\right\} .
$$

Se $x^{k+1}=x^{k}$, pare.

Destacamos que as Hipóteses 3.1.1 e 3.1.2 garantem que a função minimizada na iteração do algoritmo possui conjuntos de nível compactos, resultando na existência de mínimos. Portanto, a seqüência gerada pelo Algoritmo 3.1.3 está bem definida. Outro fato importante é que os pontos fixos deste algoritmo são exatamente os pontos de mínimo de f. Esta é uma conseqüência imediata da Hipótese 3.1.2.

\section{Análise de Convergência}

Iniciamos pela caracterização de algumas propriedades importantes de $\phi$ próximo à origem.

Lema 3.1.4. Seja $\left\{z^{k}\right\}$ uma seqüência no $\mathbb{R}^{n}$. As seguintes afirmações são equivalentes:

(a). $\phi\left(z^{k}\right) \rightarrow 0$;

(b). $z^{k} \rightarrow 0$;

(c). Existe um $\mathrm{N}>0$ tal que, para todo $\mathrm{k}>\mathrm{N}$, $\phi$ é subdiferenciável em $\mathrm{z}^{\mathrm{k}}$ e $\forall \mathrm{k}>$ $\mathrm{N}, \forall \gamma^{k} \in \partial \phi\left(z^{k}\right), \gamma^{k} \rightarrow 0$.

(d). Existe um $\mathrm{N}>0$ tal que, para todo $\mathrm{k}>\mathrm{N}$, $\phi$ é subdiferenciável em $z^{\mathrm{k}} e \forall \mathrm{k}>$ $N, \forall \gamma^{k} \in \partial \phi\left(z^{k}\right),\left\langle\gamma^{k}, z^{k}\right\rangle \rightarrow 0$.

Demonstração. 
1. (a) $\Rightarrow$ (b). Se $\phi\left(z^{k}\right) \rightarrow 0$, então $\left\{z^{k}\right\}$ é limitada, uma vez que $\phi$ possui conjuntos de nível compactos. Seja $\left\{z^{k}\right\}_{k \in \mathcal{K}}$ uma subseqüência convergente de $\left\{z^{k}\right\}$. Como $\phi$ é fechada,

$$
\phi(\bar{z}) \leq \lim _{i \in \mathcal{K}} \phi\left(z^{k}\right)=0=\min _{z \in \mathbb{R}^{n}}\{\phi(z)\} .
$$

Portando $\bar{z}$ é o ponto de mínimo de $\phi$, ou seja, $\bar{z}=0$.

2. (b) $\Rightarrow$ (c). Já que $z^{k} \rightarrow 0$, existe um $N>0$ tal que para todo $k>N, z^{k}$ está no interior do dom $\phi$, onde $\phi$ é subdiferenciável. Para $k>N$, seja $\gamma^{k} \in \partial \phi\left(z^{k}\right)$. $\left\{\gamma^{k}\right\}_{k>N}$ é limitada [37, Teorema 24.7] e, devido a semi-continuidade exterior de $\partial \phi$, todo ponto de acumulação de $\left\{\gamma^{k}\right\}_{k>N}$ é um elemento de $\partial \phi(0)=\{0\}$.

3. (c) $\Rightarrow$ (d). Basta mostrar que $\left\{z^{k}\right\}$ é limitada. Como $\gamma^{k} \rightarrow 0$ e

$$
\frac{\left\langle\gamma^{k}, z^{k}\right\rangle}{\left\|z^{k}\right\|} \geq \frac{\phi\left(z^{k}\right)-\phi(0)}{\left\|z^{k}\right\|}=\frac{\phi\left(z^{k}\right)}{\left\|z^{k}\right\|} \geq 0,
$$

segue que

$$
\frac{\phi\left(z^{k}\right)}{\left\|z^{k}\right\|} \rightarrow 0 .
$$

Portando $\left\{z^{k}\right\}$ é limitada, pois senão haveria contradição com o fato de $\phi$ possuir curvas de nível limitadas [41, Corolário 3.27].

4. (d) $\Rightarrow$ (a). Como $\gamma^{k} \in \partial \phi\left(z^{k}\right),\left\langle\gamma^{k}, z^{k}\right\rangle \geq \phi\left(z^{k}\right)-\phi(0)=\phi\left(z^{k}\right) \geq 0$. Tomando limites para $k \rightarrow \infty$ temos $\phi\left(z^{k}\right) \rightarrow 0$.

Podemos provar o seguinte teorema de convergência para o Algoritmo 3.1.3:

Teorema 3.1.5. Seja $\left\{x^{k}\right\}$ uma seqüência obtida pelo Algoritmo 3.1.3. Se $\bar{x}$ é um ponto de acumulação desta seqüência, $x^{k} \rightarrow_{\mathcal{K}} \bar{x}$, então $\bar{x}$ minimiza $f e x^{k+1} \rightarrow_{\mathcal{K}} \bar{x}$.

Demonstração. Seja $\widetilde{\alpha}$ o limite superior de $\left\{\alpha_{k}\right\}$. Para evitar tecnicalidades associadas a uma subseqüência de $\left\{\alpha_{k}\right\}$ convergindo para 0 definimos:

$$
\widetilde{x}^{k} \stackrel{\text { def }}{=} \underset{x \in \mathbb{R}^{n}}{\operatorname{argmin}}\left\{f(x)+\widetilde{\alpha} \phi\left(x-x^{k}\right)\right\} .
$$

Claramente,

$$
\begin{aligned}
f\left(\tilde{x}^{k}\right)+\widetilde{\alpha} \phi\left(\widetilde{x}^{k}-x^{k}\right) & \leq f\left(x^{k+1}\right)+\widetilde{\alpha} \phi\left(x^{k+1}-x^{k}\right) \\
f\left(x^{k+1}\right)+\alpha_{k} \phi\left(x^{k+1}-x^{k}\right) & \leq f\left(\widetilde{x}^{k}\right)+\alpha_{k} \phi\left(\widetilde{x}^{k}-x^{k}\right)
\end{aligned}
$$


Usando as desigualdades acima e $\alpha_{\mathrm{k}} \leq \widetilde{\alpha}$, concluímos que

$$
f\left(x^{k+1}\right) \leq f\left(\widetilde{x}^{k}\right) .
$$

Portanto,

$$
f\left(x^{k+1}\right)+\widetilde{\alpha} \phi\left(\widetilde{x}^{k}-x^{k}\right) \leq f\left(\widetilde{x}^{k}\right)+\widetilde{\alpha} \phi\left(\tilde{x}^{k}-x^{k}\right) \leq f\left(x^{k}\right) .
$$

Já que, pela Hipótese 3.1.1, f é limitada inferiormente, segue que

$$
0 \leq \tilde{\alpha} \phi\left(\tilde{x}^{k}-x^{k}\right) \leq \lim _{k \rightarrow \infty} f\left(x^{k}\right)-f\left(x^{k+1}\right)=0 .
$$

Assim,

$$
\phi\left(\widetilde{x}^{k}-x^{k}\right) \rightarrow 0
$$

A definição de $\widetilde{x}^{k}$ assegura que existem $\widetilde{\gamma}_{f}^{k} \in \partial f\left(\widetilde{x}^{k}\right)$ e $\widetilde{\gamma}_{\phi}^{k} \in \partial \phi\left(\widetilde{x}^{k}-x^{k}\right)$ tais que

$$
\widetilde{\gamma}_{f}^{k}+\widetilde{\alpha} \widetilde{\gamma}_{\phi}^{k}=0
$$

De (3.2) e, através do Lema 3.1.4, podemos observar que

$$
\tilde{x}^{k}-x^{k} \rightarrow 0 \text { e } \tilde{\gamma}_{f}^{k}=-\tilde{\alpha} \tilde{\gamma}_{\phi}^{k} \rightarrow 0 \text {. }
$$

Finalmente, seja $x^{k} \rightarrow_{\mathcal{K}} \bar{x}$. O resultado acima implica que $\widetilde{x}^{k} \rightarrow_{\mathcal{K}} \bar{x}$ e, devido à semicontinuidade exterior de $\partial f$,

$$
0 \in \partial f(\bar{x})
$$

Ou seja, $\bar{x}$ minimiza $f$.

Resta provar que $\left\{x^{k+1}\right\}_{k \in \mathcal{K}}$ converge para $\bar{\chi}$. Notemos que

$$
f\left(x^{k+1}\right)+\alpha_{k} \phi\left(x^{k+1}-x^{k}\right) \leq f(\bar{x})+\alpha_{k} \phi\left(\bar{x}-x^{k}\right) \leq f\left(x^{k+1}\right)+\alpha_{k} \phi\left(\bar{x}-x^{k}\right) .
$$

Conseqüentemente

$$
0 \leq \phi\left(x^{k+1}-x^{k}\right) \leq \phi\left(\bar{x}-x^{k}\right) .
$$

Como $\phi\left(\bar{x}-x^{k}\right) \rightarrow_{\mathcal{K}} 0$, seque que $\phi\left(x^{k+1}-x^{k}\right) \rightarrow_{\mathcal{K}}$ 0. O Lema 3.1.4 assegura então que $x^{k+1} \rightarrow \kappa \bar{x}$.

Podemos completar este resultado de otimalidade dos pontos de acumulação, com a convergência ao valor ótimo:

Corolário 3.1.6. Seja $\left\{x^{k}\right\}$ uma seqüência obtida pelo Algoritmo 3.1.3. Se $\left\{x^{k}\right\}$ possuir um ponto de acumulação, então

$$
f\left(x^{k}\right) \rightarrow \inf _{x \in \mathbb{R}^{n}}\{f(x)\}
$$


Demonstração. Seja $\left\{x^{k}\right\}_{k \in \mathcal{K}}$ uma subseqüência convergente de $\left\{x^{k}\right\}, x^{k} \rightarrow \mathcal{K} \bar{x}$. O Teorema 3.1.5 mostra que $\bar{x}$ é um minimizador de $f$ e que $x^{k+1} \rightarrow \mathcal{K} \bar{x}$. Seja $\gamma_{\phi}^{k+1} \in$ $\partial \phi\left(x^{k+1}-x^{k}\right)$ tal que $\exists \gamma_{f}^{k+1} \in \partial f\left(x^{k+1}\right),-\alpha_{k} \gamma_{\phi}^{k+1}=\gamma_{f}^{k+1}$.

Como $x^{k+1}-x^{k} \rightarrow_{\mathcal{K}} 0$, temos que $\gamma_{\phi}^{k+1} \rightarrow_{\mathcal{K}} 0$. Portando,

$$
f(\bar{x}) \leq \lim _{k \rightarrow \mathcal{K}^{\infty}} f\left(x^{k+1}\right) \leq \lim _{k \rightarrow \mathcal{K}^{\infty}} f(\bar{x})+\alpha_{k}\left\langle\gamma_{\phi}^{k+1}, \bar{x}-x^{k+1}\right\rangle=f(\bar{x}) .
$$

Já que $\left\{f\left(x^{k}\right)\right\}$ é não-crescente e limitada, ela é convergente para $f(\bar{x})$, o mínimo de f.

\subsection{Uma versão inexata do $\phi$-MPP}

Na seção anterior, apresentamos resultados de convergência para o Algoritmo 3.1.3 caso os sub-problemas sejam resolvidos com exatidão. Surge naturalmente a pergunta: é possível resolver estes sub-problemas aproximadamente e mesmo assim obter resultados de convergência, como foi feito no capítulo anterior? O próximo teorema é uma resposta afirmativa a esta questão.

Teorema 3.2.1. Suponha que as Hipóteses 3.1.1 e 3.1.2 são válidas. Seja $\sigma \in[0,1)$ $e\left\{\alpha_{k}\right\}$ uma seqüência de reais positivos limitada superiormente por $\widetilde{\alpha}$. Seja $\left\{x^{k}\right\}$ uma seqüência gerada iterativamente tal que para $\gamma_{f}^{k+1} \in \partial f\left(x^{k+1}\right), \gamma_{\phi}^{k+1} \in \partial \phi\left(x^{k+1}-x^{k}\right)$ e $e^{k+1} \stackrel{\text { def }}{=} \gamma_{f}^{k+1}+\alpha_{k} \gamma_{\phi}^{k+1}$,

$$
\left\|e^{k+1}\right\| \leq \sigma \frac{\alpha_{k}\left\langle\gamma_{\phi}^{k+1}, x^{k+1}-x^{k}\right\rangle}{\left\|x^{k+1}-x^{k}\right\|}
$$

Suponha ainda que uma das seguintes condições valem:

(a). $\left\{\alpha_{k}\right\}$ é limitada inferiormente por um certo $\widehat{\alpha}>0$,

(b). A seqüência $\Delta x^{\mathrm{k}} \stackrel{\text { def }}{=} \mathrm{x}^{\mathrm{k}+1}-\mathrm{x}^{\mathrm{k}}$ está contida em um compacto, $\mathrm{K}$, no interior de $\operatorname{dom} \phi$,

(c). Existe um $\in>0$ tal que para todo $x \in \mathbb{R}^{n}$ e $\gamma_{\phi} \in \partial \phi(x)$

$$
\frac{\left\langle\gamma_{\phi}, x\right\rangle}{\left\|\gamma_{\phi}\right\|\|x\|} \geq \epsilon \text {. }
$$

Então, $\gamma_{f}^{k} \rightarrow 0$. Em particular, todo ponto de acumulação da seqüência $\left\{x^{k}\right\}$ é um minimizador de $\mathrm{f}$. 
Demonstração. Vamos supor, por contradição, que exista uma subseqüência $\left\{\gamma_{f}^{k}\right\}_{k \in \mathcal{K}}$ tal que $\gamma_{f}^{k} A_{\mathcal{K}} 0$. Como $0<\alpha_{k-1} \leq \tilde{\alpha}$, nós podemos assumir, sem perda de generalidade, que existe $\bar{\alpha} \geq 0, \alpha_{k-1} \rightarrow_{\mathcal{K}} \bar{\alpha}$.

De posse desta notação, temos:

$$
\begin{aligned}
f\left(x^{k-1}\right) & \geq f\left(x^{k}\right)+\left\langle\gamma_{f}^{k}, x^{k-1}-x^{k}\right\rangle \\
& =f\left(x^{k}\right)+\left\langle\alpha_{k-1} \gamma_{\phi}^{k}, x^{k}-x^{k-1}\right\rangle+\left\langle\gamma_{f}^{k}+\alpha_{k-1} \gamma_{\phi}^{k}, x^{k-1}-x^{k}\right\rangle \\
& \geq f\left(x^{k}\right)+\alpha_{k-1}\left\langle\gamma_{\phi}^{k}, x^{k}-x^{k-1}\right\rangle-\left\|\gamma_{f}^{k}+\alpha_{k-1} \gamma_{\phi}^{k}\right\|\left\|x^{k}-x^{k-1}\right\| \\
& \geq f\left(x^{k}\right)+(1-\sigma) \alpha_{k-1}\left\langle\gamma_{\phi}^{k}, x^{k}-x^{k-1}\right\rangle .
\end{aligned}
$$

A última desigualdade é uma conseqüência do critério de aceitação (3.3). Portanto,

$$
\alpha_{k-1}\left\langle\gamma_{\phi}^{k}, x^{k}-x^{k-1}\right\rangle \rightarrow 0
$$

Por outro lado, usando mais uma vez (3.3):

$$
\left\|\gamma_{f}^{k}\right\| \leq(1+\sigma) \alpha_{k-1}\left\|\gamma_{\phi}^{k}\right\|
$$

Consideramos três possibilidades:

A primeira está associada a $\bar{\alpha}>0$, o que é certamente válido sob (a). Neste caso, (3.4) implica que

$$
\left\langle\gamma_{\phi}^{k}, x^{k}-x^{k-1}\right\rangle \rightarrow \mathcal{K} 0 .
$$

Usando Lema 3.1.4 e (3.5), segue que $\gamma_{f}^{k} \rightarrow_{\mathcal{K}} 0$.

Os próximos casos estão associados a $\bar{\alpha}=0$. Se a condição (b) for válida, então $\forall k \in \mathcal{K}, \gamma_{\phi}^{k} \in \partial \phi(K)$ e, portanto, $\left\{\gamma_{\phi}^{k}\right\}_{k \in \mathcal{K}}$ é limitada e $\alpha_{k-1} \rightarrow_{\mathcal{K}} 0$. Usando (3.5), concluímos que $\gamma_{f}^{k} \rightarrow \mathcal{K} 0$.

Sob a hipótese (c), usando (3.4), temos que

$$
\alpha_{k-1}\left\|\gamma_{\phi}^{k}\right\|\left\|x^{k}-x^{k-1}\right\| \epsilon \rightarrow \mathcal{K} 0 .
$$

Vamos mais uma vez provar que $\gamma_{f}^{k} \rightarrow \mathcal{K} 0$. Caso contrário, (3.5) assegura que existe uma subseqüência $\mathcal{K}^{\prime} \subset \mathcal{K}$ e um $\delta>0$ tal que $\forall k \in \mathcal{K}^{\prime}, \alpha_{k-1}\left\|\gamma_{\phi}^{k}\right\|>\delta$. Então, $\left\|x^{k}-x^{k-1}\right\| \rightarrow \mathcal{K}^{\prime} 0$. Mas, usando o Lema 3.1.4, isto implicaria que $\alpha_{k-1} \gamma_{\phi}^{k} \rightarrow \mathcal{K}^{\prime} 0$, uma contradição.

Em todos os casos, a existência de uma subseqüência tal que $\gamma_{f}^{k} 厶_{\mathcal{K}} 0$ é negada, portanto, $\gamma_{f}^{k} \rightarrow 0$. Deste modo, a semi-continuidade exterior de $\partial f$ implica que todo ponto de acumulação de $\left\{x^{k}\right\}$ minimiza $f$. 


\subsection{Métodos de multiplicadores}

Nesta seção, nós vamos usar a versão exata do Algoritmo 3.1.3 para apresentar uma classe de métodos de multiplicadores para programação convexa que é uma generalização da classe $\mathrm{P}_{\mathrm{E}}^{+}$discutida em [8]. Infelizmente, o critério de aceitação de uma solução aproximada do passo proximal dado por (3.3) não se traduz a uma condição facilmente verificável no contexto de métodos de multiplicadores. A questão da resolução aproximada dos sub-problemas de métodos de multiplicadores será tratada no Capítulo 6, no qual apresentaremos algumas classes de métodos proximais que resolvem parcialmente esta limitação.

Mais uma vez, queremos resolver o problema de programação convexa com restrições (1.2). Para isto, vamos aplicar o Algoritmo 3.1.3 à solução do problema dual (1.3). Neste caso obteríamos a seguinte iteração:

$$
\lambda^{k+1}=\underset{\lambda \geq 0}{\operatorname{argmax}}\left\{F(\lambda)-\alpha_{k} \phi\left(\lambda-\lambda^{k}\right)\right\} .
$$

Usando o Teorema 2.2.6, sabemos que podemos realizar estes passos através do seguinte método de multiplicadores:

Algoritmo 3.3.1. Método de Multiplicadores Baseado em Funções Estritamente Convexas

Considere o problema primal (1.2) e seu respectivo dual lagrangiano.

Seja $\widetilde{\alpha}$ um número real estritamente positivo e $\phi: \mathbb{R}^{m} \rightarrow(-\infty, \infty]$ uma função que satisfaz a Hipótese 3.1.2.

1. Inicialização: Comece com $\lambda^{1}$ viável dual.

2. Iteração:

- Escolha $0<\alpha_{k} \leq \widetilde{\alpha}$ e calcule uma solução, $\chi^{k+1}$, do problema:

$$
\begin{array}{ll}
\min & f(x)+\alpha_{k} P\left(\frac{g(x)}{\alpha_{k}}, \lambda^{k}\right) \\
\text { s.a } \quad x \in \mathbb{R}^{n},
\end{array}
$$

para,

$$
P\left(u, \lambda^{k}\right) \stackrel{\text { def }}{=}\left(\phi\left(\cdot-\lambda^{k}\right)+\delta_{\mathbb{R}_{+}^{m}}(\cdot)\right)^{*}(u) .
$$

- Faça

$$
\lambda^{k+1} \stackrel{\text { def }}{=} \nabla_{u} P\left(\frac{g\left(x^{k+1}\right)}{\alpha_{k}}, \lambda^{k}\right)
$$

Se $\lambda^{k+1}=\lambda^{k}$, pare. $^{2}$

\footnotetext{
${ }^{2}$ Neste caso $\lambda^{k}$ é uma solução do problema dual, pois é um ponto fixo do Algoritmo 3.1.3.
} 
Desta forma, a seqüência $\left\{\lambda^{k}\right\}$ é a mesma obtida pelo Algoritmo 3.1.3. Exemplos de algumas penalidades que podem ser obtidas a partir de diferentes escolhas de $\phi$ são apresentados na Figura 3.1.

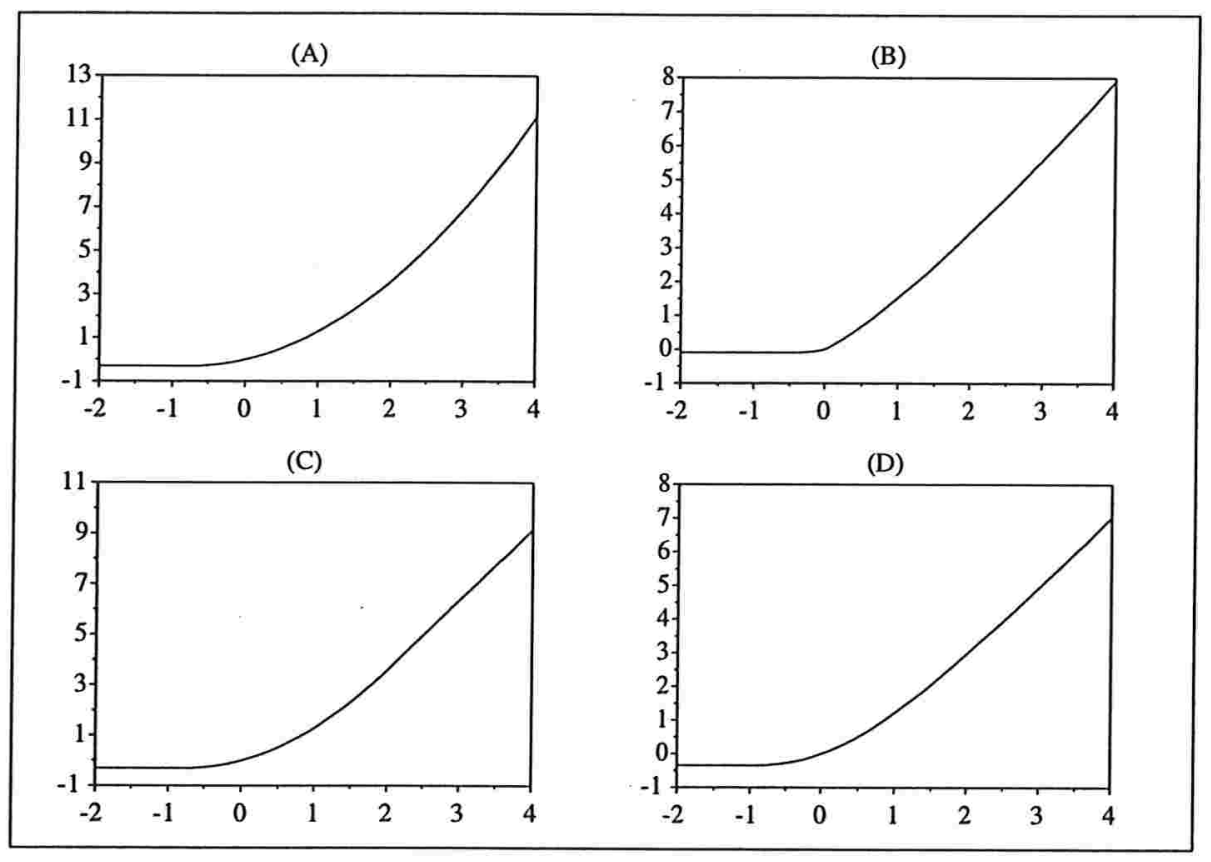

Figura 3.1: Exemplos de penalidade: (A) A penalidade clássica, usada no método de multiplicadores de Hestennes e Powell. (B) A penalidade $P(u, \lambda)=u \lambda+\frac{3}{4} x^{\frac{4}{3}}$, obtida a partir de $\phi(u)=\frac{1}{4} u^{4}$. (C) A função de regularização usada aqui é $\phi(u)=\frac{1}{2} u^{2}$ restrita a um intervalo contendo a origem. Fora deste intervalo $\phi$ é igualada a $+\infty$. Isto faz com que a penalidade seja afim acima de um certo valor positivo (2, neste exemplo). (D) Esta penalidade foi obtida usando a função de regularização $\phi(u)=-\ln (\cos (u))$. Como $\phi^{\prime}(u)=\tan (u)$, a penalidade terá derivada limitada e converge assintoticamente para uma função afim.

Para garantir a obtenção de soluções duais, é preciso que $\left\{\lambda^{k}\right\}$ esteja contida em um compacto. Isto será verdade se os conjuntos de nível de $\mathrm{F}$ forem compactos, já que o Algoritmo 3.1.3 é um método de descida. Como vimos no Teorema 1.2.5, a compacidade dos condutos de nível de F é equivalente a validade da condição de qualificação de Slater.

\section{A seqüência primal}

Na definição do método de multiplicadores, assumimos que todos os sub-problemas primais que serão resolvidos têm solução. Isto não é sempre verdade, como foi discutido 
após a prova do Lema 2.2.9. Entretanto, este mesmo lema apresenta condições para que estas soluções existam: basta que o problema primal (1.2) possua conjunto de soluções compacto e não-vazio e que $\operatorname{dom} \phi=\mathbb{R}^{m}$, pois desta forma para todo $\lambda \geq 0$, $\operatorname{dom} \phi(\cdot-\lambda) \supset \mathbb{R}_{++}^{m}$.

Finalmente, apresentamos o principal teorema de convergência para seqüência primal calculada pelo Algoritmo 3.3.1:

Teorema 3.3.2. Seja $\left\{\left(x^{k}, \lambda^{k}\right)\right\}$ uma seqüência calculada pelo Algoritmo 3.3.1. Se o problema primal satisfaz a condição de qualificação de Slater, então

$$
\begin{gathered}
\limsup _{k \rightarrow \infty} g\left(x^{k}\right) \leq 0, \\
\lim _{k \rightarrow \infty}\left\langle\lambda^{k}, g\left(x^{k}\right)\right\rangle=0,
\end{gathered}
$$

e $\mathrm{f}\left(\mathrm{x}^{\mathrm{k}}\right)$ converge para o valor ótimo do problema primal, $v(0)$. Portanto, todo ponto de acumulação da seqüência primal é uma solução do problema primal (1.2).

Demonstração. A limitação da seqüência dual e o Teorema 3.1 .5 implicam que $\lambda^{k}-$ $\lambda^{k-1} \rightarrow 0$. Portanto, $\forall \gamma_{\phi}^{k} \in \partial \phi\left(\lambda^{k}-\lambda^{k-1}\right), \gamma_{\phi}^{k} \rightarrow 0$.

Usando definição do algoritmo, sabemos que:

$$
\lambda^{k}=\nabla_{u} P\left(\frac{g\left(x^{k}\right)}{\alpha_{k-1}}, \lambda^{k-1}\right) \Leftrightarrow g\left(x^{k}\right) \in \partial\left(\alpha_{k-1} \phi\left(\cdot-\lambda^{k-1}\right)+\delta_{\mathbb{R}_{+}^{m}}(\cdot)\right)\left(\lambda^{k}\right) .
$$

Isto é equivalente a existência de subgradientes $\gamma_{\phi}^{k} \in \partial \phi\left(\lambda^{k}-\lambda^{k-1}\right)$ e $\gamma_{\delta}^{k} \in \partial \delta_{\mathbb{R}_{+}^{m}}\left(\lambda^{k}\right)$, tais que

$$
g\left(x^{k}\right)=\alpha_{k-1} \gamma_{\phi}^{k}+\gamma_{\delta}^{k}
$$

Como $\gamma_{\delta}^{k} \leq 0$, temos:

$$
\limsup _{k \rightarrow \infty} g\left(x^{k}\right) \leq \lim _{k \rightarrow \infty} \alpha_{k-1} \gamma_{\phi}^{k}=0
$$

Além disto, a definição de $\gamma_{\delta}^{k}$ implica que $\left\langle\gamma_{\delta}^{k}, \lambda^{k}\right\rangle=0$, e assim

$$
\lim _{k \rightarrow \infty}\left\langle\lambda^{k}, g\left(x^{k}\right)\right\rangle=\lim _{k \rightarrow \infty}\left\langle\lambda^{k}, \alpha_{k} \gamma_{\phi}^{k}\right\rangle=0 \text {. }
$$

A última igualdade é uma conseqüência de $\gamma_{\phi}^{k} \rightarrow 0$ e da limitação de $\left\{\lambda^{k}\right\}$ e $\left\{\alpha_{k}\right\}$.

Por fim, vamos prova que $f\left(x^{k}\right) \rightarrow v(0)$. A definição de $P, \lambda^{k}$, e [37, Teorema 23.5] implicam que

$$
\alpha_{k-1} P\left(\frac{g\left(x^{k}\right)}{\alpha_{k-1}}, \lambda^{k-1}\right)+\alpha_{k-1} \phi\left(\lambda^{k}-\lambda^{k-1}\right)+\delta_{\mathbb{R}_{+}^{m}}\left(\lambda^{k}\right)=\left\langle\lambda^{k}, g\left(x^{k}\right)\right\rangle .
$$


Além disto, lembrando da Proposição 2.2.5, também temos que

$$
\begin{aligned}
f\left(x^{k}\right)+\alpha_{k-1} P\left(\frac{g\left(x^{k}\right)}{\alpha_{k-1}}, \lambda^{k-1}\right) & =\min _{x}\left\{f(x)+\alpha_{k-1} P\left(\frac{g(x)}{\alpha_{k-1}}, \lambda^{k-1}\right)\right\} \\
& =\max _{\lambda \geq 0}\left\{F(\lambda)-\alpha_{k-1} \phi\left(\lambda-\lambda^{k-1}\right)\right. \\
& =F\left(\lambda^{k}\right)-\alpha_{k-1} \phi\left(\lambda^{k}-\lambda^{k-1}\right) .
\end{aligned}
$$

Logo, usando (3.7), segue:

$$
F\left(\lambda^{k}\right)=f\left(x^{k}\right)+\left\langle\lambda^{k}, g\left(x^{k}\right)\right\rangle
$$

ou seja, $x^{k}$ é um mínimo da função lagrangiana com $\lambda^{k}$ como multiplicador. ${ }^{3}$ Usando o Corolário 3.1.6 e o teorema forte de dualidade, nós concluímos que:

$$
v(0)=\lim _{k \rightarrow \infty} F\left(\lambda^{k}\right)=\lim _{k \rightarrow \infty} f\left(x^{k}\right)+0 .
$$

Encerramos esta seção apresentando uma condição que assegura a limitação da seqüência primal e a conseqüente existência de pontos de acumulação, que serão soluções de (1.2).

Corolário 3.3.3. Suponha que o problema primal satisfaça a condição de qualificação de Slater e que $\left\{x^{k}\right\}$ seja uma seqüência primal obtida pelo Algoritmo 3.3.1. Se o conjunto de soluçôes do primal for não-vazio e limitado, então $\left\{x^{k}\right\}$ é limitada.

Demonstração. O Teorema 3.3.2 implica que $\left\{g\left(x^{k}\right)\right\}$ é limitada superiormente. Portanto, $\left\{x^{k}\right\}$ está contida em uma intersecção de conjuntos de nível das restrições.

Supomos, por contradição, que $\left\{x^{k}\right\}$ é ilimitada. Uma vez que o conjunto de soluções de (1.2) é compacto e não-vazio, $f\left(x^{k}\right) \rightarrow \infty$, pois, caso contrário, pelo menos um dos pontos de acumulação de $\frac{x^{k}}{\left\|x^{k}\right\|}$ seria uma direção de recessão comum à função objetivo e todas as restrições. Porém, isto contraria $f\left(x^{k}\right) \rightarrow v(0) \in \mathbb{R}$, provado no Teorema 3.3.2.

\footnotetext{
${ }^{3}$ Essa argumentação rebuscada faz-se necessária por não conhecermos um teorema do tipo regra da cadeia que possa ser usado na composição de P e g. Resultados do tipo [41, Teorema 10.6, 10.19, 10.49] possuem condições restritivas como a separabilidade de $P$, ou diferenciabilidade de $\mathrm{g}$, ou $P$ estritamente crescente.
} 


\section{Distâncias Coercivas}

Na década de 90 , observou-se um interesse crescente por métodos proximais baseados em distâncias coercivas $[16,45,19,28,27,1,6,20,4,5]$. A principal vantagem deste tipo de regularização, quando comparada ao quadrado da norma euclidiana ou às regularizações descritas no Capítulo 3, é a possibilidade da resolução de problemas com restrições simples (como caixas ou poliedros) através de passos proximais irrestritos. ${ }^{1}$ Isto se deve ao fato de a distância ter, ao mesmo tempo, funções de regularização e de barreira. Outro ponto positivo é a possibilidade da obtenção de métodos de multiplicadores baseados em penalidades infinitamente diferenciáveis, o que não ocorre no $\phi$-MPP.

Este capítulo busca, através do uso de técnicas semelhantes àquelas presentes nos capítulos anteriores, apresentar uma ampla classe de regularizações separáveis e coercivas para a solução de problemas com restrições do tipo caixa (1.1) e (1.4). Vamos provar a convergência, no sentido de subseqüências, dos respectivos métodos proximais e, em seguida, analisaremos o caso de otimização e métodos de multiplicadores. Este capítulo é baseado em [42] e possui uma estreita ligação com os recentes artigos de Auslender, Teboulle e Ben-Tiba $[4,5]$ nos quais regularizações baseadas em $\varphi$-divergência são estudadas. Nós iremos colocar este artigos em um contexto mais amplo que engloba também distâncias de Bregman.

\subsection{Regularizações Coercivas Separáveis}

Vamos nos dedicar ao estudo de métodos proximais baseados em regularizações separáveis e coercivas tais que:

\footnotetext{
${ }^{1} \mathrm{Na}$ verdade, métodos baseados em distâncias de Bregman são apresentados conceitualmente para restrições convexas quaisquer. Entretanto, a maior parte das distâncias de Bregman conhecidas explicitamente lidam com restrições simples como caixas, bolas ou poliedros.
} 
Hipótese 4.1.1. Para $i=1, \ldots, n$, as funções $d_{i}: \mathbb{R} \times\left(a_{i}, b_{i}\right) \rightarrow(-\infty, \infty]$ possuem as seguintes propriedades:

(a). Para todo $y_{i} \in\left(a_{i}, b_{i}\right), d_{i}\left(\cdot, y_{i}\right)$ é estritamente convexa, fechada e atinge mínimo em $y_{i}$. Ainda, int dom $d_{i}\left(\cdot, y_{i}\right)=\left(a_{i}, b_{i}\right)$.

(b). $d_{i}$ é diferenciável em $\left(a_{i}, b_{i}\right) \times\left(a_{i}, b_{i}\right)$ com relação ao seu primeiro argumento e esta derivada parcial é contínua em todo ponto da forma $\left(x_{i}, x_{i}\right) \in\left(a_{i}, b_{i}\right) \times$ $\left(a_{i}, b_{i}\right)$. Ainda, lembrando de (1.8), denotamos

$$
d_{i}^{\prime}\left(x_{i}, y_{i}\right) \stackrel{\text { def }}{=} \frac{\partial d_{i}}{\partial x_{i}}\left(x_{i}, y_{i}\right)
$$

(c). Para todo $y_{i} \in\left(a_{i}, b_{i}\right), \operatorname{rge~}_{i}^{\prime}\left(\cdot, y_{i}\right)=\mathbb{R}$.

(d). Existem $L, \epsilon>0$ tais que, sempre que $-\infty<a_{i}<y_{i} \leq x_{i}<a_{i}+\epsilon$ ou $b_{i}-\epsilon<$ $x_{i} \leq y_{i}<b_{i}<+\infty$ temos que $\left|d_{i}^{\prime}\left(x_{i}, y_{i}\right)\right| \leq L\left|x_{i}-y_{i}\right|$.

De uma forma geral, tais hipóteses são naturais em métodos proximais. A hipótese de estrita convexidade está presente em praticamente todos os métodos proximais que conhecemos, a exigência de diferenciabilidade também é comum. Escolhemos considerar que a imagem $d_{i}^{\prime}\left(\cdot, y_{i}\right)$ é a reta real por simplicidade. Ela é usada somente no Lema 4.2.3, que prova que a seqüência proximal, descrita pelo Algoritmo 4.2.1, está bem definida e contida no interior da caixa B. Existem outras condições, menos restritivas para as regularizações, que garantem a boa definição da seqüência proximal, como as hipóteses apresentadas no Apêndice A. Porém, estas condições restringem o tipo de problemas variacionais que podemos resolver. ${ }^{2}$

Por fim, a Hipótese 4.1.1(d) é nova na teoria de métodos proximais generalizados, entretanto ela não é muito restritiva na prática. Em particular, mostraremos a seguir que é possível obter regularizações baseadas em distâncias de Bregman e $\varphi$-divergências para as quais esta condição é facilmente verificável e válida na grande maioria dos exemplos que conhecemos.

\section{Mudança de Escala}

Para obter regularizações d que obedeçam à Hipótese 4.1.1, vamos usar uma técnica que denominamos mudança de escala. Se $\widetilde{\mathrm{d}}(\cdot, \cdot)$, é uma componente de uma distância

\footnotetext{
${ }^{2}$ Por exemplo, se a desigualdade variacional vier de um problema de minimização, basta supor que as regularizações agem como uma barreira, não deixando os iterados proximais saírem de int $B$.
} 
generalizada separável ${ }^{3}$, definimos uma nova componente:

$$
d(x, y) \stackrel{\text { def }}{=} \frac{\widetilde{d}(x, y)}{\widetilde{d}^{\prime \prime}(y, y)} .
$$

Pode-se interpretar esta fórmula como uma tentativa de recuperar o comportamento do termo regularizador clássico em uma vizinhança do centro, forçando-se sua hessiana a coincidir com a identidade.

\section{Distâncias de Bregman}

As distâncias de Bregman foram introduzidas em [11] e aplicadas a métodos de ponto proximal em [16, 18, 19] e vários artigos posteriores. A construção destas distâncias usa uma função estritamente convexa auxiliar, $h: \mathbb{R} \rightarrow(-\infty, \infty]$, int dom $h=(a, b)$. Se y é o centro, então a distância de um outro ponto ao centro é a diferença entre $h$ e sua aproximação linear em $y$, ou seja, $\tilde{d}(x, y) \stackrel{\text { def }}{=} h(x)-h(y)-h^{\prime}\left(y_{i}\right)(x-y)$. Também é possível obter distâncias não separáveis estendendo esta fórmula para o caso multi-dimensional. Mas neste texto trataremos apenas o caso separável, que é o mais corriqueiro.

Como foi discutido anteriormente, estamos interessados em usar como regularização a distância obtida a partir de $\widetilde{d}$ através da mudança de escala dada por (4.1):

$$
d(x, y) \stackrel{\text { def }}{=} \frac{h(x)-h(y)-h^{\prime}(y)(x-y)}{h^{\prime \prime}(y)} .
$$

As propriedades a seguir garantem que a Hipótese 4.1.1 seja válida para d:

Hipótese 4.1.2. Seja $h: \mathbb{R} \rightarrow(-\infty, \infty]$, int dom $h=(a, b)$ :

(a). h é convexa, fechada e duas vezes diferenciável em ( $a, b)$, com segunda derivada estritamente positiva. Isto implica que $h$ é estritamente convexa.

(b). rge $h^{\prime}=\mathbb{R}$.

(c). Existem $M>0$ e $\epsilon>0$ tais que se $-\infty<a<y \leq x<a+\epsilon$ ou se $b-\epsilon<x \leq$ $y<b<+\infty$, então $\left|h^{\prime}(x)-h^{\prime}(y)\right| \leq M h^{\prime \prime}(y)|x-y|$.

\footnotetext{
${ }^{3}$ Como as regularizações atuam separadamente em cada coordenada, nós podemos fazer a apresentação dos exemplos assumindo que as distâncias atuam em $\mathbb{R}$ ao invés de $\mathbb{R}^{n}$, evitando a presença de índices para as componentes das distâncias e das variáveis e empregando, assim, uma notação mais simples.
} 
A Hipótese 4.1.2(c) é nova na teoria de funções de Bregman e corresponde a Hipótese 4.1.1(d), uma vez que $\widetilde{d}^{\prime \prime}(x, y)=h^{\prime \prime}(x)$. Vamos analisá-la com mais cuidado. Para isto, consideremos o caso no qual a é finito. Como $\lim _{x \downarrow a} h^{\prime}(x)=-\infty$, sabemos que $h^{\prime \prime}(x)$ deve ser ilimitada superiormente a medida que $x$ se aproxima de a. Para violar a 4.1.2(c), $h^{\prime \prime}(x)$ teria que oscilar de forma cada vez mais rápida para $x \downarrow$ a, o que configuraria um comportamento inesperado para uma regularização. De uma forma geral, podemos afirmar que as funções de Bregman usualmente propostas na literatura têm um comportamento ainda mais regular, obedecendo à hipótese do seguinte lema:

Lema 4.1.3. Se existe um $\epsilon>0$ tal que $\mathrm{h}^{\prime \prime}$ é não-crescente em $(\mathrm{a}, \mathrm{a}+\epsilon) \cap \mathbb{R} e \mathrm{~h}^{\prime \prime} e^{\prime}$ não-decrescente, em $(\mathrm{b}-\epsilon, \mathrm{b}) \cap \mathbb{R}$, então a Hipótese 4.1.2(c) é satisfeita.

Demonstração. Suponha que $a \in \mathbb{R}$ e $\operatorname{sejam} x, y \in(a, a+\epsilon)$ tais que $y<x$. Então,

$$
\begin{aligned}
\left|h^{\prime}(x)-h^{\prime}(y)\right| & =\int_{y}^{x} h^{\prime \prime}(z) d z \\
& \leq h^{\prime \prime}(y)|x-y| .
\end{aligned}
$$

Portando, a Hipótese 4.1.2(c) vale com $M=1$. O caso b $\in \mathbb{R}$ é análogo.

Exemplos de funções que obedecem à Hipótese 4.1.2 e às condições do lema acima são:

- $h(x)=x \log (x)$, para $(a, b)=\mathbb{R}_{++;}$

- $h(x)=x^{\alpha}-x^{\beta}$, se $\alpha \in(1,2], \beta \in(0,1)$ e $(a, b)=\mathbb{R}_{++}$;

- $h(x)=x \log \left(e^{x}-1\right)$, para $(a, b)=\mathbb{R}_{++}$;

- $h(x)=\frac{1}{2} x^{2}$, para $(a, b)=\mathbb{R}$;

- $h(x)=\frac{1}{x}+\frac{1}{2} x^{2}$, para $(a, b)=\mathbb{R}_{++} ;$

- $h(x)=-\log (x)+\frac{1}{2} x^{2}, \operatorname{para}(a, b)=\mathbb{R}_{++;} ;$

- $h(x)=x-x^{\beta}+\frac{1}{2} x^{2}$, se $\beta \in(0,1)$ e $(a, b)=\mathbb{R}_{++\cdot}$ s

Devemos chamar atenção para o fato de que não há nenhuma hipótese sobre o comportamento da função de Bregman nos extremos do intervalo (a,b). Esta condição, que é comum para distâncias de Bregman, não é necessária em nossa análise.

\footnotetext{
$\S^{\S}$ No caso de otimização, como discutido no Apêndice $A$, poderíamos empregar $h(x)$ igual a $\frac{1}{x}$ ou $-\log (x)$ ou $x-x^{\beta}$, respectivamente.
} 
$\varphi$-divergências

As regularizações conhecidas por $\varphi$-divergências aparecem freqüentemente em textos sobre pontos proximais, por exemplo em [28], e mais recentemente em [6, 4, 5]. Nestes trabalhos, a caixa B é o ortante positivo do $\mathbb{R}^{n}$. Uma função estritamente convexa, $\varphi: \mathbb{R}_{+} \rightarrow(-\infty, \infty]$, é usada na definição da distância $\widetilde{d}$, através da fórmula:

$$
\tilde{d}(x, y) \stackrel{\text { def }}{=} y \varphi\left(\frac{x}{y}\right) \text {. }
$$

Mais uma vez definimos d através da mudança de escala (4.1). Como $\widetilde{d}^{\prime \prime}(y, y)=$ $1 / y \varphi^{\prime \prime}(1)$, temos que $d$ possui a forma

$$
d(x, y) \stackrel{\text { def }}{=} \frac{y^{2}}{\varphi^{\prime \prime}(1)} \varphi\left(\frac{x}{y}\right)
$$

Neste caso, a Hipótese 4.1.1 transforma-se em:

Hipótese 4.1.4. Seja $\varphi: \mathbb{R} \rightarrow(-\infty, \infty]$, int dom $\varphi=\mathbb{R}_{++}$:

(a). $\varphi$ é estritamente convexa e fechada;

(b). $\varphi$ é duas vezes diferenciável no $\mathbb{R}_{++}$;

(c). $\varphi(1)=\varphi^{\prime}(1)=0, \varphi^{\prime \prime}(1)>0$;

(d). $\operatorname{rge} \varphi^{\prime}=\mathbb{R}$

(e). Existe um $L>0$ tal que $\varphi^{\prime}(t) \leq L \varphi^{\prime \prime}(t)(t-1), \forall t \geq 1$.

Com pequenas variações, esta hipótese aparece em vários artigos, em particular [6, $4,5]$, que também são os primeiros trabalhos a sugerir o uso de termos regularizadores baseados em (4.4), substituindo a definição anterior (4.3). Desta forma os resultados deste capítulo podem ser vistos como uma extensão de $[6,4,5]$.

Alguns exemplos de funções que satisfazem a Hipótese 4.1.4 presentes em [5]:

- $\varphi(x)=x \log (x)-x+1$

- $\varphi(x)=x^{-1}+x-2$;

- $\varphi(x)=2(\sqrt{x}-1)^{2}+\frac{1}{2}(x-1)^{2} ;$ 
- $\varphi(x)=-\log (x)+x-1+\frac{1}{2}(x-1)^{2}$.

É interessante notar que, em [28], os autores afirmam que a única interseç̧ão entre $\varphi$-divergências e distâncias de Bregman é a distância conhecida como Kullback-Leibler:

$$
d(x, y)=x \log \frac{x}{y}+y-x .
$$

Porém, se fizermos a mudança de escala, veremos que as distâncias dadas por (4.4) e por (4.2) coincidem em vários outros casos. Dedicaremos o Apêndice B ao estudo da relação entre essas classes de distâncias.

Claramente, a Hipótese 4.1.4 é uma releitura da Hipótese 4.1.1 no contexto de $\varphi$ divergências. $\mathrm{O}$ único ponto que merece um pouco mais de atenção é a equivalência entre as condições 4.1.1(d) e 4.1.4(e). Isto é objeto do lema:

Lema 4.1.5. Seja $d_{i}$ uma distância definida por (4.4). Então as Condições 4.1.1(d) e 4.1.4(e) são equivalentes.

Demonstração. Observemos primeiro que:

$$
\begin{aligned}
d_{i}^{\prime}\left(x_{i}, y_{i}\right) & =\frac{y_{i}}{\varphi^{\prime \prime}(1)} \varphi^{\prime}\left(\frac{x_{i}}{y_{i}}\right) \\
d_{i}^{\prime \prime}\left(x_{i}, y_{i}\right) & =\frac{1}{\varphi^{\prime \prime}(1)} \varphi^{\prime \prime}\left(\frac{x_{i}}{y_{i}}\right) .
\end{aligned}
$$

Logo,

$$
d_{i}^{\prime \prime}\left(y_{i}, y_{i}\right)=1
$$

Portanto, a Hipótese 4.1.1(d) se reduz a:

$$
\exists L, \epsilon>0: \quad 0<y_{i} \leq x_{i}<\epsilon \quad \Rightarrow \quad \varphi^{\prime}\left(\frac{x_{i}}{y_{i}}\right) \leq \frac{L \varphi^{\prime \prime}(1)}{y_{i}}\left(x_{i}-y_{i}\right) .
$$

Tomando $x_{i} \in(0, \epsilon)$ e fazendo com que $y_{i}$ percorra o intervalo $\left(0, x_{i}\right]$, obtemos:

$$
\exists \mathrm{L}>0: \quad \varphi^{\prime}(\mathrm{x}) \leq \mathrm{L} \varphi^{\prime \prime}(1)(x-1) \forall x \geq 1 .
$$

Reciprocamente, se (4.6) é valida, o mesmo ocorre com (4.5) para qualquer $\epsilon>0$.

\footnotetext{
"Mais uma vez, no caso de otimização, estes dois últimos exemplos poderiam ser simplificados para $2(\sqrt{x}-1)^{2} e-\log (x)+x-1$.
} 


\subsection{O Método Proximal com Distâncias Coercivas Separáveis}

Uma vez caracterizada a classe de regularizações de nosso interesse, podemos apresentar o respectivo método proximal para resolução do problema de desigualdade variacional (1.4) e analisar sua convergência.

Algoritmo 4.2.1. Método Proximal com Distâncias Coercivas para Desigualdades Variacionais

Considere o problema da desigualdade variacional (1.4).

Seja $\widetilde{\alpha}$ um real positivo e $\left\{\beta_{k}\right\}$ uma sequência somável de reais positivos. Sejam $d_{i}$, $i=1, \ldots, n$, regularizações que obedeçam a Hipótese 4.1.1. Defina $D \stackrel{\text { def }}{=} \sum_{i=1}^{n} d_{i}$.

1. Inicialização: Comece $\operatorname{com} x^{1} \in \operatorname{int} B$;

2. Iteração: Escolha $0<\alpha_{i}^{k} \leq \widetilde{\alpha}, i=1, \ldots, n$ e encontre $x^{k+1} \in \operatorname{int} B \cap \operatorname{dom} T$ e $e^{k+1}$ tais que

$$
\begin{gathered}
e^{k+1} \in T\left(x^{k+1}\right)+\operatorname{diag}\left(\alpha^{k}\right) \nabla_{1} D\left(x^{k+1}, x^{k}\right), \\
\left|e_{i}^{k+1}\right| \leq \alpha_{i}^{k}\left|d_{i}^{\prime}\left(x_{i}^{k+1}, x_{i}^{k}\right)\right|+\beta_{k+1}, i=1, \ldots, n .
\end{gathered}
$$

Se $x^{k+1}=x^{k}$, pare. ${ }^{6}$

Para provar a convergência do Algoritmo 4.2.1, vamos lançar mão de uma hipótese adicional que, apesar de sua artificialidade aparente, será facilmente verificada em várias especializações deste algoritmo. Desta forma, temporariamente, o método proximal descrito acima deve ser visto como um algoritmo abstrato que auxiliará a resolução de problemas de programação convexa e de desigualdades variacionais, temas da próxima seção e do Capítulo 5.

Começamos por introduzir uma notação que será usada constantemente no restante do texto:

$$
\gamma^{\mathrm{k}} \stackrel{\text { def }}{=} e^{k}-\operatorname{diag}\left(\alpha^{k-1}\right) \nabla_{1} \mathrm{D}\left(x^{k}, x^{k-1}\right) \text {. }
$$

Usando (4.7) segue que $\gamma^{k} \in T\left(x^{k}\right)$ para todo $k \geq 1$.

A hipótese aparentemente artificial que necessitamos é:

Hipótese 4.2.2. Se $x^{k} \rightarrow_{\mathcal{K}} \bar{x}$, então, para cada $i=1, \ldots, n$, ou $\gamma_{i}^{k} \rightarrow_{\mathcal{K}} 0$ ou existe um conjunto infinito $\mathcal{K}^{\prime} \subseteq \mathcal{K}$ tal que $x_{i}^{k-1} \rightarrow \mathcal{K}^{\prime} \bar{x}_{i}$.

\footnotetext{
${ }^{6}$ É fácil observar que, neste caso $x^{k}$ é uma solução da desigualdade variacional com $T\left(x^{k}\right)=0$.
} 


\section{Análise de Convergência}

Esta seção dedica-se a análise de convergência da seqüência $\left\{x^{k}\right\}$ gerada pelo Algoritmo 4.2.1. Ela está organizada da seguinte maneira: primeiro provamos que a existência do iterados. Em seguida mostramos que todos os possíveis limites da seqüência $\left\{\gamma^{k}\right\}$, definida em (4.9), possuem uma estrutura de sinais que caracteriza as soluções do problema da desigualdade variacional (1.4). Isto é feito no Lema 4.2.4. Finalmente, observamos que as subseqüências de $\left\{\gamma^{k}\right\}$ associadas a subseqüências convergentes de $\left\{x^{k}\right\}$ são limitadas. Isto permitirá provar que todo ponto de acumulação de $\left\{x^{k}\right\}$ é uma solução do problema de (1.4).

Lema 4.2.3. Seja $H: \mathbb{R}^{n} \rightarrow(-\infty, \infty]$ uma função estritamente convexa, fechada tal que $\operatorname{dom} \partial \mathrm{H}=\operatorname{int} \mathrm{B}, \mathrm{H}$ é diferenciável em int $\mathrm{B}$ e rge $\partial \mathrm{H}=\mathbb{R}^{n}$. Então existe $\bar{x} \in \operatorname{int} \mathrm{B} \cap \operatorname{dom} \mathrm{T}$ tal que

$$
0 \in \mathrm{T}(\bar{x})+\nabla H(\bar{x}) .
$$

Isto implica que a seqüência $\left\{x^{k}\right\}$ computada pelo Algoritmo 4.2.1 está bem definida.

Demonstração. Para provar este resultado vamos mostrar que a equação generalizada

$$
0 \in T(\bar{x})+N_{B}(\bar{x})+\partial H(\bar{x})
$$

possui soluções. Em seguida mostraremos que estas soluções estão em int B, onde H é diferenciável e $N_{B}$ é identicamente nula.

Observamos primeiro que, como dom $\mathrm{T} \cap \operatorname{int} B \neq \emptyset$, podemos usar o [41, Corolário 12.44] e concluir que $T+N_{B}+\partial H$ é um operador monótono maximal.

Como a imagem de $\partial \mathrm{H}$ é o $\mathbb{R}^{n},[35$, Proposição 3] nos diz que o mesmo ocorre com $\mathrm{T}+\mathrm{N}_{\mathrm{B}}+\partial \mathrm{H}$. Portanto este operador possui zeros.

Suponha que algum destes zeros está na fronteira da caixa. Neste caso, $\mathrm{H}$, deve ser subdiferenciável na fronteira de $\mathrm{B}$, o que contraria dom $\partial \mathrm{H}=\operatorname{int} \mathrm{B}$. Logo os zeros de $T+N_{B}+D\left(\cdot, x^{k}\right)$ estão em int B e, desta forma, a equação

$$
0 \in T\left(x^{k+1}\right)+\nabla_{1} D\left(x^{k+1}, x^{k}\right)
$$

possui soluções.

Assim, o resultado acima prova que o passo iterativo do Algoritmo 4.2.1 pode ser realizado com $e^{k+1}=0$. Para ver isto, basta tomar $H(x) \stackrel{\text { def }}{=} \sum_{i=1}^{n} \alpha_{i}^{k} d_{i}\left(x_{i}, x_{i}^{k}\right), \forall x \in$ $\mathbb{R}^{n}$.

Analisamos a seguir a estrutura de sinais dos possíveis limites da seqüência $\left\{\gamma^{k}\right\}$, definida em (4.9). 
Lema 4.2.4. Seja $\left\{x^{k}\right\}$ uma seqüência obtida pelo Algoritmo 4.2.1 e assuma que a Hipótese 4.2.2 é válida. Se $\bar{x} \in \mathbb{R}^{n}$ é um ponto de acumulação de $\left\{x^{k}\right\}, x^{k} \rightarrow_{\mathcal{K}} \bar{x}$, então para $i=1, \ldots, n$,

$$
\begin{aligned}
\lim _{k \rightarrow \kappa^{\infty}} \gamma_{i}^{k}=0 & \text { se } \bar{x}_{i} \in\left(a_{i}, b_{i}\right) \\
\liminf _{k \rightarrow \kappa^{\infty}} \gamma_{i}^{k} \geq 0 & \text { se } \bar{x}_{i}=a_{i} \\
\limsup _{k \rightarrow \kappa^{\infty}} \gamma_{i}^{k} \leq 0 & \text { se } \bar{x}_{i}=b_{i} .
\end{aligned}
$$

Demonstração. Para cada i, vamos considerar os três casos possíveis:

Primeiro, supomos que $\bar{x}_{i} \in\left(a_{i}, b_{i}\right)$. Com o objetivo de obter uma contradição, vamos assumir que $\gamma_{i}^{k} A_{\mathcal{K}} 0$. Então, usando a Hipótese 4.2.2, deve existir um conjunto infinito de índices $\mathcal{K}^{\prime} \subseteq \mathcal{K}$ e um escalar $\zeta>0$ tal que para todo $k \in \mathcal{K}^{\prime},\left|\gamma_{i}^{k}\right| \geq \zeta$ e $x_{i}^{k-1} \rightarrow \mathcal{K}^{\prime} \bar{x}_{i}$. Logo,

$$
\begin{aligned}
\left|\gamma_{i}^{k}\right| & =\left|e_{i}^{k}-\alpha_{i}^{k-1} d_{i}^{\prime}\left(x_{i}^{k}, x_{i}^{k-1}\right)\right| \\
& \leq \alpha_{i}^{k-1}\left|d_{i}^{\prime}\left(x_{i}^{k}, x_{i}^{k-1}\right)\right|+\left|e_{i}^{k}\right| \\
& \leq 2 \alpha_{i}^{k-1}\left|d_{i}^{\prime}\left(x_{i}^{k}, x_{i}^{k-1}\right)\right|+\beta_{k} \\
& \leq 2 \widetilde{\alpha}\left|d_{i}^{\prime}\left(x_{i}^{k}, x_{i}^{k-1}\right)\right|+\beta_{k} \\
& {\overrightarrow{\mathcal{K}^{\prime}}}^{2} \widetilde{\alpha}\left|d_{i}^{\prime}\left(\bar{x}_{i}, \bar{x}_{i}\right)\right|+0 \\
& =0 .
\end{aligned}
$$

O que contradiz $\left|\gamma_{i}^{k}\right|>\zeta, k \in \mathcal{K}^{\prime}$.

Agora, vamos analisar o caso $\bar{x}_{i}=a_{i}$. Assumimos que $\lim \inf _{k \rightarrow \kappa} \infty \gamma_{i}^{k}<0$ para obter uma contradição. A Hipótese 4.2 .2 garante que existem um escalar $\zeta>0$ e um conjunto infinito $\mathcal{K}^{\prime} \subseteq \mathcal{K}$ tal que para todo $k \in \mathcal{K}^{\prime}, \gamma_{i}^{k} \leq-\zeta$ e $x_{i}^{k-1} \rightarrow \mathcal{K}^{\prime} \bar{x}_{i}$. Portanto,

$$
\begin{aligned}
\zeta & \leq\left|\gamma_{i}^{k}\right| \\
& =\left|e_{i}^{k}-\alpha_{i}^{k-1} d_{i}^{\prime}\left(x_{i}^{k}, x_{i}^{k-1}\right)\right| \\
& \leq 2 \alpha_{i}^{k-1}\left|d_{i}^{\prime}\left(x_{i}^{k}, x_{i}^{k-1}\right)\right|+\beta_{k} \\
& \leq 2 \widetilde{\alpha}\left|d_{i}^{\prime}\left(x_{i}^{k}, x_{i}^{k-1}\right)\right|+\beta_{k} .
\end{aligned}
$$

Seja $\epsilon$ dado pela Hipótese 4.1.1(d). Se existir um conjunto infinito de índices $\mathcal{K}^{\prime \prime} \subseteq \mathcal{K}^{\prime}$ tal que $x_{i}^{k-1} \leq x_{i}^{k} \leq a_{i}+\epsilon$ para $k \in \mathcal{K}^{\prime \prime}$, nós podemos usar esta hipótese para concluir que:

$$
\begin{aligned}
\zeta & \leq 2 \widetilde{\alpha}\left|d_{i}^{\prime}\left(x_{i}^{k}, x_{i}^{k-1}\right)\right|+\beta_{k} \\
& \leq 2 \widetilde{\alpha} L\left|x_{i}^{k}-x_{i}^{k-1}\right|+\beta_{k} \\
& \underset{\mathcal{K}^{\prime \prime}}{ } 0
\end{aligned}
$$


uma vez que $x^{k-1} \rightarrow \mathcal{K}^{\prime} \bar{x}_{i}$ e $\beta_{k} \rightarrow 0$; porém isto contradiz $\zeta>0$. Segue que, $x_{i}^{k} \leq x_{i}^{k-1}$ para $k \in \mathcal{K}^{\prime}$ suficientemente grande.

Como $d_{i}\left(\cdot, x_{i}^{k-1}\right)$ atinge mínimo em $x_{i}^{k-1}, x_{i}^{k} \leq x_{i}^{k-1}$ implica que $d_{i}^{\prime}\left(x_{i}^{k}, x_{i}^{k-1}\right) \leq 0$. Deste modo,

$$
\begin{aligned}
\gamma_{i}^{k} & =e_{i}^{k}-\alpha_{i}^{k-1} d_{i}^{\prime}\left(x_{i}^{k}, x_{i}^{k-1}\right) \\
& \geq \alpha_{i}^{k-1}\left|d_{i}^{\prime}\left(x_{i}^{k}, x_{i}^{k-1}\right)\right|-\left|e_{i}^{k}\right| \\
& \geq-\beta_{k} \\
& >-\zeta
\end{aligned}
$$

para $k \in \mathcal{K}^{\prime}$ suficientemente grande. Uma contradição com $\gamma_{i}^{k} \leq-\zeta<0, k \in \mathcal{K}^{\prime}$.

$\mathrm{O}$ caso $\bar{x}_{i}=b_{i}$ é análogo ao caso $\bar{x}_{i}=a_{i}$.

Lema 4.2.5. Seja $\left\{x^{k}\right\}$ uma seqüência obtida pelo Algoritmo 4.2.1 e assuma que a Hipótese 4.2.2 é válida. Seja $\bar{x}$ um ponto de acumulação de $\left\{x^{k}\right\}, x^{k} \rightarrow \mathcal{K} \bar{\chi}$. Então, $\left\{\gamma^{\mathrm{k}}\right\}_{\mathrm{k} \in \mathcal{K}}$ é limitada.

Demonstração. A Definição 1.2.6 garante que existe $\tilde{x} \in \operatorname{dom} T \cap \operatorname{int} B$. Seja $\tilde{\gamma} \in T(\tilde{x})$. Então a monotonicidade de $T$ implica que, para todo $k \geq 0$,

$$
0 \leq\left\langle x^{k}-\tilde{x}, \gamma^{k}-\tilde{\gamma}\right\rangle=\sum_{i=1}^{n}\left(x_{i}^{k}-\tilde{x}_{i}\right)\left(\gamma_{i}^{k}-\tilde{\gamma}_{i}\right) .
$$

Vamos mostrar que a ilimitação de $\left\{\gamma^{k}\right\}_{k \in \mathcal{K}}$ gera uma contradição com esta desigualdade para $k$ suficientemente grande.

Se $\left\{\gamma^{k}\right\}_{k \in \mathcal{K}}$ for ilimitada, existe um subconjunto infinito $\mathcal{K}^{\prime} \subseteq \mathcal{K}$ tal que $\left\{\gamma^{k}\right\}_{\mathcal{K}^{\prime}}$ converge em $\overline{\mathbb{R}}$, com pelo menos uma componente $\left\{\gamma_{i}^{k}\right\}_{\mathcal{K}^{\prime}}$ ilimitada. O Lema 4.2.4 implica que cada uma destas coordenadas ilimitadas, deve obedecer a:

$$
\begin{gathered}
\gamma_{i}^{k} \rightarrow \mathcal{K}^{\prime}+\infty, \text { se } \bar{x}_{i}=a_{i} \\
\text { ou } \\
\gamma_{i}^{k} \rightarrow \mathcal{K}^{\prime}-\infty, \text { se } \bar{x}_{i}=b_{i} .
\end{gathered}
$$

Portanto, cada componente ilimitada de $\left\{\gamma^{k}\right\}_{\mathcal{K}^{\prime}}$, tem a propriedade que

$$
\begin{aligned}
& \left(x_{i}^{k}-\tilde{x}_{i}\right)\left(\gamma_{i}^{k}-\tilde{\gamma}_{i}\right) \rightarrow \mathcal{K}^{\prime}\left(a_{i}-\tilde{x}_{i}\right)(+\infty)=-\infty \\
& \left(x_{i}^{k}-\tilde{x}_{i}\right)\left(\gamma_{i}^{k}-\tilde{\gamma}_{i}\right) \rightarrow \mathcal{K}^{\prime}\left(b_{i}-\tilde{x}_{i}\right)(-\infty)=-\infty
\end{aligned}
$$

Por outro lado, caso $\left\{\gamma_{i}^{k}\right\} \mathcal{K}^{\prime}$ seja limitada, $\left(x_{i}^{k}-\widetilde{x}_{i}\right)\left(\gamma_{i}^{k}-\widetilde{\gamma}_{i}\right)$ também o é. Portanto, para $k \in \mathcal{K}^{\prime} \subseteq \mathcal{K}$ suficientemente grande, $\left\langle x^{k}-\tilde{x}, \gamma^{k}-\widetilde{\gamma}\right\rangle$ seria negativo, uma contradição com (4.11). 
Agora é possível enunciar e provar o principal teorema de convergência para o Algoritmo 4.2.1.

Teorema 4.2.6. Seja $\left\{x^{k}\right\}$ uma seqüência obtida pelo Algoritmo 4.2.1 e assuma que a Hipótese 4.2.2 é válida. Todo ponto de acumulaçẫo de $\left\{x^{k}\right\}$ é uma solução da desigualdade variacional (1.4).

Demonstração. Seja $\bar{x}$ um ponto de acumulação de $\left\{x^{k}\right\}, x^{k} \rightarrow \mathcal{K} \bar{x}$. Seja $\left\{\gamma^{k}\right\}_{k \in \mathcal{K}}$ dada por 4.9. O Lema 4.2.5 diz que esta subseqüência é limitada. Logo, deve haver um subconjunto infinito de índices $\mathcal{K}^{\prime} \subseteq \mathcal{K}$ tal que $\gamma^{k} \rightarrow \mathcal{K}^{\prime} \bar{\gamma} \in \mathbb{R}^{\mathrm{n}}$. Como $\mathrm{T}$ é semicontínuo exterior $[41,12.8(\mathrm{~b})]$, temos que $\bar{\gamma} \in \mathrm{T}(\bar{x})$. Lema 4.2.4 prova então que:

$$
\begin{array}{ll}
\bar{\gamma}_{i}=0 & \text { se } \bar{x}_{i} \in\left(a_{i}, b_{i}\right), \\
\bar{\gamma}_{i} \geq 0 & \text { se } \bar{x}_{i}=a_{i}, \\
\bar{\gamma}_{i} \leq 0 & \text { se } \bar{x}_{i}=b_{i},
\end{array}
$$

e isto é equivalente a $0 \in T(\bar{x})+N_{B}(\bar{x})$.

De posse deste resultado, passamos a analisar casos nos quais as hipóteses do Teorema 4.2.6 são facilmente verificáveis. Em particular, a condição que se mostra mais difícil é a Hipótese 4.2.2, já que as outras dependem apenas de uma escolha adequada das regularizações e da possibilidade de resolver o passo proximal aproximadamente, com precisão controlada pelo critério de aceitação dado em (4.8).

\subsection{Métodos Proximais com Distâncias Coercivas Separáveis Aplicados à Otimização}

Analisaremos aqui o Algoritmo 4.2.1 para a solução do problema programação convexa em caixas (1.1). A estrutura extra que este problema possui, em particular a presença de uma função objetivo, permite uma fácil verificação da Hipótese 4.2.2.

Iniciamos com uma releitura da condição dom $T \cap$ int $B \neq \emptyset$, presente na Definição 1.2.6, para o caso de otimização. Neste caso, ela se resume a dom $\partial f \cap \operatorname{int} B \neq \emptyset$. Porém, como f é convexa, própria e fechada, ela é subdiferenciável no interior relativo de seu domínio efetivo. Assim, já que B tem interior não vazio podemos simplificar esta condição à $\operatorname{dom} f \cap \operatorname{int} B \neq \emptyset$.

O Algoritmo 4.2.1 torna-se:

Algoritmo 4.3.1. Método Proximal com Distâncias Coercivas para Otimização

Considere o problema de minimização em caixa definido em 1.2.1. Assuma que $\operatorname{dom} f \cap \operatorname{int} B \neq \emptyset$. 
Seja $\widetilde{\alpha}$ um real positivo e $\sigma \in[0,1]$. Sejam $d_{i}, i=1, \ldots, n$, regularizações que obedeçam a Hipótese 4.1.1. Defina $\mathrm{D} \stackrel{\text { def }}{=} \sum_{i=1}^{n} d_{i}$.

1. Inicialização: Comece $\operatorname{com} x^{1} \in \operatorname{int} B$;

2. Iteração: Escolha $0<\alpha_{i}^{k} \leq \widetilde{\alpha}, i=1, \ldots, n$, e encontre $x^{k+1} \in \operatorname{int} B \cap \operatorname{dom} \partial f$ e $e^{k+1}$ tais que

$$
\begin{aligned}
& e^{k+1} \in \partial f\left(x^{k+1}\right)+\operatorname{diag}\left(\alpha^{k}\right) \nabla_{1} D\left(x^{k+1}, x^{k}\right), \\
& \left|e_{i}^{k+1}\right| \leq \sigma \alpha_{i}^{k}\left|d_{i}^{\prime}\left(x_{i}^{k+1}, x_{i}^{k}\right)\right|, i=1, \ldots, n .
\end{aligned}
$$

Se $x^{k+1}=x^{k}$, pare.

\section{Análise de Convergência}

Seja $\left\{x^{k}\right\}$ uma seqüência computada pelo Algoritmo 4.3.1. Lembrando do Teorema 4.2.6, notamos que para provar a otimalidade dos pontos de acumulação de $\left\{x^{k}\right\}$ basta provar que a Hipótese 4.2.2 é automaticamente satisfeita, desta forma, passamos a esta tarefa.

Como em (4.9), definimos:

$$
\gamma^{k} \stackrel{\text { def }}{=} e^{k}-\operatorname{diag}\left(\alpha^{k-1}\right) \nabla_{1} D\left(x_{i}^{k}, x_{i}^{k-1}\right)
$$

Lembramos que esta definição implica que $\gamma^{k} \in \partial f\left(x^{k}\right)$.

Começamos a análise demonstrando um lema técnico:

Lema 4.3.2. Seja $\left\{x^{k}\right\}$ uma seqüência obtida pelo Algoritmo 4.3.1. Para todo $k \geq 2$ e todo $i=1, \ldots, n, \gamma_{i}^{k}\left(x_{i}^{k-1}-x_{i}^{k}\right) \geq 0$.

Demonstração. Temos,

$$
\begin{aligned}
\gamma_{i}^{k}\left(x_{i}^{k-1}-x_{i}^{k}\right) & =\underbrace{\left(e_{i}^{k}-\alpha_{i}^{k-1} d_{i}^{\prime}\left(x_{i}^{k}, x_{i}^{k-1}\right)\right)\left(x_{i}^{k-1}-x_{i}^{k}\right)}_{\geq 0} \\
& \geq \underbrace{-\alpha_{i}^{k-1} d_{i}^{\prime}\left(x_{i}^{k}, x_{i}^{k-1}\right)\left(x_{i}^{k-1}-x_{i}^{k}\right)}_{i}-\left|e_{i}^{k}\right|\left|x_{i}^{k-1}-x_{i}^{k}\right| \\
& =\alpha_{i}^{k-1}\left(\left|d_{i}^{\prime}\left(x_{i}^{k}, x_{i}^{k-1}\right)\right|-\frac{1}{\alpha_{i}^{k-1}}\left|e_{i}^{k}\right|\right)\left|x_{i}^{k-1}-x_{i}^{k}\right| \\
& \geq(1-\sigma) \alpha_{i}^{k-1}\left|d_{i}^{\prime}\left(x_{i}^{k}, x_{i}^{k-1}\right)\right|\left|x_{i}^{k-1}-x_{i}^{k}\right| \\
& \geq 0 .
\end{aligned}
$$


Lema 4.3.3. Seja $\left\{\chi^{k}\right\}$ uma seqüência obtida pelo Algoritmo 4.3.1. A seqüência $\left\{f\left(x^{k}\right)\right\}$ é não-crescente e portanto convergente se f for limitada inferiormente em B. Neste caso,

$$
\left|\gamma_{i}^{k}\right|\left|x_{i}^{k-1}-x_{i}^{k}\right| \rightarrow 0, \forall i=1, \ldots, n .
$$

Demonstração. Como $\gamma^{k} \in \partial f\left(x^{k}\right)$,

$$
f\left(x^{k-1}\right) \geq f\left(x^{k}\right)+\left\langle\gamma^{k}, x^{k-1}-x^{k}\right\rangle=f\left(x^{k}\right)+\sum_{i=1}^{n} \gamma_{i}^{k}\left(x_{i}^{k-1}-x_{i}^{k}\right),
$$

e assim a tese resulta do fato de $\gamma_{i}^{k}\left(x_{i}^{k-1}-x_{i}^{k}\right)$ ser não-negativo.

Observamos que, se $f$ for limitada inferiormente em B, a Hipótese 4.2.2 é uma conseqüência imediata deste lema. Portanto, o Teorema 4.2.6 garante a otimalidade dos pontos de acumulação da seqüência $\left\{x^{k}\right\}$. No caso de otimização, este resultado ainda pode ser melhorado:

Teorema 4.3.4. Seja $\left\{x^{k}\right\}$ uma seqüência obtida pelo Algoritmo 4.3.1. Se f for limitada inferiormente em $\mathrm{B}$ e $\left\{\mathrm{x}^{\mathrm{k}}\right\}$ tiver um ponto de acumulação, então $\left\{f\left(x^{k}\right)\right\}$ converge para o infimo de $\mathrm{f}$ em $\mathrm{B}$ e, conseqüentemente, todo ponto de acumulação de $\left\{x^{k}\right\}$ será uma solução do problema de programação convexa na caixa B (1.1).

Demonstraçãa. Seja $\bar{x}$ um ponto de acumulação de $\left\{x^{k}\right\}, x^{k} \rightarrow \mathcal{K} \bar{x}$. O Teorema 4.2.6 mostra que $\bar{x}$ minimiza $f$ em B. Deste modo, como $\gamma^{k} \in \partial f\left(x^{k}\right)$, sabemos que

$$
\min _{x \in B}\{f(x)\}=f(\bar{x}) \geq f\left(x^{k}\right)+\sum_{i=1}^{n} \gamma_{i}^{k}\left(\bar{x}_{i}-x_{i}\right) .
$$

Lembrando que $x^{k} \rightarrow_{\mathcal{K}} \bar{x}$ e que $\left\{\gamma^{k}\right\}_{\mathcal{K}}$ é limitada (Lema 4.2.5), podemos tomar o limite em $\mathcal{K}$ acima para concluir que

$$
\min _{x \in B}\{f(x)\}=\lim _{k \rightarrow \kappa \infty} f\left(x^{k}\right) .
$$

Finalmente, como $\left\{f\left(x^{k}\right)\right\}$ é não-crescente, pelo Lema 4.3.3, temos:

$$
\lim _{k \rightarrow \infty} f\left(x^{k}\right)=\min _{x \in B}\{f(x)\} .
$$

Devemos ainda destacar que, como o Algoritmo 4.3.1 é um método de descida, $\left\{x^{k}\right\}$ será limitada sempre que o conjunto solução, ou qualquer outra interseç̧ão entre um conjunto de nível de $f$ e $B$, for limitado. De fato, o conjunto solução é um conjunto de nível de $f+\delta_{B}$, e portanto sua limitação é equivalente à limitação de todos os outros conjuntos de nível desta função [41, Proposição 3.23]. 


\section{Métodos de Multiplicadores}

Nesta seção, voltamos nossa atenção aos métodos de multiplicadores que podem ser obtidos a partir do Algoritmo 4.3.1. Ou seja, desejamos resolver o problema de programação convexa com restrições (1.2) através da aplicação dos métodos proximais com distâncias coercivas e resolução exata dos sub-problemas ${ }^{7}$ à resolução do problema dual (1.3). Usando o Teorema 2.2.6, obtemos o seguinte método:

\section{Algoritmo 4.3.5. Método de Multiplicadores com Distâncias Coercivas}

Considere o problema primal (1.2) e seu respectivo dual lagrangiano. Suponha ainda que exista um ponto viável dual estritamente positivo.

Seja $\tilde{\alpha}$ um real positivo. Sejam $d_{i}: \mathbb{R} \times\left(a_{i},+\infty\right) \rightarrow(-\infty, \infty], i=1, \ldots, n$, regularizações que obedeçam a Hipótese 4.1.1, tais que $a_{i} \leq 0$.

1. Inicialização: Comece $\operatorname{com} \lambda^{1}>0$.

2. Iteração:

- Escolha $0<\alpha_{i}^{k} \leq \tilde{\alpha}, i=1, \ldots, m$, e calcule uma solução, $x^{k+1}$, do problema:

$$
\begin{array}{ll}
\min & f(x)+\sum_{i=1}^{m} \alpha_{i}^{k} P_{i}\left(\frac{g_{i}(x)}{\alpha_{i}^{k}}, \lambda_{i}^{k}\right) \\
\text { s.a } & x \in \mathbb{R}^{n},
\end{array}
$$

para,

$$
P_{i}\left(u_{i}, \lambda_{i}^{k}\right) \stackrel{\text { def }}{=}\left(d_{i}\left(\cdot, \lambda_{i}^{k}\right)+\delta_{\mathbb{R}_{+}}(\cdot)\right)^{*}\left(u_{i}\right)
$$

- Faça

$$
\lambda_{i}^{k+1} \stackrel{\text { def }}{=} \frac{\partial P_{i}}{\partial u_{i}}\left(\frac{g_{i}\left(x^{k+1}\right)}{\alpha_{i}^{k}}, \lambda_{i}^{k}\right), i=1, \ldots, m .
$$

Se $\lambda^{k+1}=\lambda^{k}$, pare.

Mais uma vez, a limitação da seqüência de multiplicadores é garantida caso o problema primal obedeça à condição de qualificação de Slater (ver Teorema 1.2.5) e a otimalidade dos seus pontos de acumulação é uma conseqüência do Teorema 4.3.4.

\footnotetext{
${ }^{7} \mathrm{~A}$ análise de métodos de multiplicadores com resolução aproximada dos sub-problemas será feita no Capítulo 6.
} 


\section{A seqüência primal}

Vamos procurar provar que o teorema de convergência dos iterados primais apresentado para os métodos de multiplicadores baseados no $\phi$-MPP também é válido para o Algoritmo 4.3.1. Este tipo de resultado é interessante uma vez que ele mostra que é possível garantir a viabilidade dos pontos de acumulação primal mesmo se usarmos regularizações baseadas em distâncias de Bregman, estendendo os resultados obtidos para $\varphi$-divergência em $[6,5]$. Enunciamos então o resultado que desejamos demonstrar:

Teorema 4.3.6. Seja $\left\{\left(x^{k}, \lambda^{k}\right)\right\}$ uma seqüência calculada pelo Algoritmo 4.3.5. Se o problema primal satisfaz a condição de qualificação de Slater, então

$$
\begin{aligned}
& \limsup _{k \rightarrow \infty} g\left(x^{k}\right) \leq 0, \\
& \lim _{k \rightarrow \infty}\left\langle\lambda^{k}, g\left(x^{k}\right)\right\rangle=0,
\end{aligned}
$$

e $f\left(x^{k}\right)$ converge para o valor ótimo do problema primal, $v(0)$. Portanto, todo ponto de acumulação da seqüência primal é uma solução do problema primal (1.2).

Demonstração. Já sabemos, graças ao Teorema 4.3 .4 e a Condição de Slater, que a seqüência de multiplicadores é limitada e que $F\left(\lambda^{k}\right) \rightarrow v(0)$. Analisemos a seqüência primal.

A definição de $\lambda^{k}$ garante que existem $\zeta_{i}^{k} \in \partial \delta_{\mathbb{R}_{+}}\left(\lambda_{i}^{k}\right), i=1, \ldots, m$ tais que

$$
g_{i}\left(x^{k}\right)=\alpha_{i}^{k-1} d_{i}^{\prime}\left(\lambda_{i}^{k}, \lambda_{i}^{k-1}\right)+\zeta_{i}^{k} .
$$

Desta forma, se $\gamma^{k}$ é o vetor definido em (4.9), temos $\gamma_{i}^{k}=-g_{i}\left(x^{k}\right)+\zeta_{i}^{k}$.

O Lema 4.2.4 garante então que, para toda subseqüência convergente da seqüência de multiplicadores, $\lambda^{k} \rightarrow_{\mathcal{K}} \bar{\lambda},-g_{i}\left(x^{k}\right)+\zeta_{i}^{k}$ obedece à:

$$
\begin{array}{r}
0=\lim _{k \rightarrow \kappa^{\infty}}\left(g_{i}\left(x^{k}\right)-\zeta_{i}^{k}\right)=\lim _{k \rightarrow \kappa^{\infty}} g_{i}\left(x^{k}\right) \text { se } \bar{\lambda}_{i}>0, \\
0 \geq \limsup _{k \rightarrow \kappa^{\infty}}\left(g_{i}\left(x^{k}\right)-\zeta_{i}^{k}\right) \geq \limsup _{k \rightarrow \kappa^{\infty}} g_{i}\left(x^{k}\right) \text { se } \bar{\lambda}_{i}=0 .
\end{array}
$$

Como $\left\{\lambda^{k}\right\}$ é limitada, concluímos facilmente que

$$
\limsup _{k \rightarrow \infty} g_{i}\left(x^{k}\right) \leq 0, i=1, \ldots, n \text {. }
$$

Agora vamos provar que

$$
\lim _{k \rightarrow \infty}\left\langle\lambda^{k}, g\left(x^{k}\right)\right\rangle=0
$$


Para isto, vamos supor, com objetivo de obter uma contradição, que para algum $i=$ $1, \ldots, n$, temos $\left|\lambda_{i}^{k} g_{i}\left(x^{k}\right)\right| \not \nrightarrow 0$. Neste caso, já que $\left\{\lambda^{k}\right\}$ é limitada e positiva, devem existir um conjunto infinito de índices $\mathcal{K} \subset \mathbb{N}, \bar{\lambda} \geq 0$ e um real $\epsilon>0$ tais que $\lambda^{k} \rightarrow \mathcal{K} \bar{\lambda}$ e $\forall k \in \mathcal{K},\left|\lambda_{i}^{k} g_{i}\left(x^{k}\right)\right|>\epsilon$. Usando as Equações (4.14) e (4.15), observamos que isto só é possível se $g_{i}\left(x^{k}\right) \rightarrow \mathcal{K}-\infty$. Isto não é possível, pois o Lema 4.2 .5 garante que $\left\{-g_{i}\left(x^{k}\right)+\zeta_{i}^{k}\right\}_{\mathcal{K}}$ é uma seqüência limitada, logo $g_{i}\left(x^{k}\right) \rightarrow \mathcal{K}-\infty$ implica que $\zeta_{i}^{k} \rightarrow \mathcal{K}$ $-\infty$. Conseqüentemente $\lambda_{i}^{k}$ deve ser 0 infinitas vezes em $\mathcal{K}$, o que contrariaria $\forall k \in$ $\mathcal{K},\left|\lambda_{i}^{k} g_{i}\left(x^{k}\right)\right|>\epsilon$.

Finalmente, resta mostrar que $f\left(x^{k}\right)$ converge para o valor ótimo do problema primal (e dual), $v(0)$. Para isto, usando a fórmula de atualização do multiplicador $\lambda^{k} \mathrm{e}$ [41, Proposição 11.3], observamos que para cada $i=1, \ldots, n$ :

$$
\alpha_{i}^{k-1} P_{i}\left(\frac{g_{i}\left(x^{k}\right)}{\alpha_{i}^{k-1}}, \lambda_{i}^{k}\right)+\alpha_{i}^{k-1} d_{i}\left(\lambda_{i}^{k}, \lambda_{i}^{k-1}\right)=\lambda_{i}^{k} g_{i}\left(x^{k}\right) .
$$

Por outro lado, as definições de $x^{k}$ e a Proposição 2.2.5 e o Teorema 2.2.6 implicam que:

$$
f\left(x^{k}\right)+\sum_{i=1}^{m} \alpha_{i}^{k} P_{i}\left(\frac{g_{i}(x)}{\alpha_{i}^{k}}, \lambda_{i}^{k}\right)=F\left(\lambda^{k}\right)-\sum_{i=1}^{m} \alpha_{i}^{k} d_{i}\left(\lambda_{i}^{k}, \lambda_{i}^{k-1}\right) .
$$

Portanto,

$$
f\left(x^{k}\right)+\left\langle\lambda^{k}, g\left(x^{k}\right)\right\rangle=F\left(\lambda^{k}\right) .
$$

Como $\left\langle\lambda^{k}, g\left(x^{k}\right)\right\rangle \rightarrow 0$ e $F\left(\lambda^{k}\right) \rightarrow v(0)$ segue que $f\left(x^{k}\right)$ também converge para o valor ótimo do problema primal.

Um fato que deve ser destacado é que a existência dos iterados primais está garantida sempre que o problema primal possuir conjunto de soluções não vazio e compacto. Isto se deve ao Lema 2.2 .9 e à condição que $\left(a_{i}, b_{i}\right) \supset \mathbb{R}_{++}$, garantindo que, para todo $\lambda \in \mathbb{R}_{+}^{m}, \operatorname{dom} \sum_{i=1}^{M} d_{i}\left(\cdot, \lambda_{i}\right) \supset \mathbb{R}_{++}$.

Resta-nos apenas apresentar um resultado que assegure a limitação da seqüência primal:

Corolário 4.3.7. Suponha que o problema primal satisfaça a condição de qualificação de Slater e que $\left\{x^{k}\right\}$ seja uma seqüência primal obtida pelo Algoritmo 4.3.5. Se o conjunto de soluções do primal for não-vazio e limitado, então $\left\{x^{k}\right\}$ é limitada.

Demonstração. A prova deste corolário é igual à prova do Corolário 3.3.3, e, por este motivo, será omitida. 


\section{Regularizações Duplas}

No capítulo anterior, introduzimos um método proximal conceitual, Algoritmo 4.2.1, para resolver o problema da desigualdade variacional. ${ }^{1}$ Para garantir sua convergência, fizemos uso da Hipótese $4.2 .2^{2}$ que é, aparentemente, difícil de ser verificada sem que façamos novas suposições sobre o problema que desejamos resolver ou sobre o método que queremos aplicar.

Neste capítulo, apresentaremos uma forma de assegurar a validade da Hipótese 4.2.2 que depende apenas da escolha das regularizações e outros parâmetros do algoritmo. A nossa estratégia é baseada na idéia de combinar um termo coercivo com outro quadrático, como feito recentemente em [4, 5]. Deste modo, é possível recuperar a Fejér monotonicidade da seqüência proximal com relação ao conjunto de soluções, que foi perdida com a mudança de escala. Neste sentido, mais uma vez, este capítulo pode ser visto como uma extensão dos resultados apresentados em $[4,5]$.

Usaremos a seguinte classe de distâncias generalizadas:

Definição 5.1.1. Seja $v$ um número real estritamente maior que 1. Para $i=1, \ldots, n$, seja $d_{i}: \mathbb{R} \times\left(a_{i}, b_{i}\right) \rightarrow(-\infty, \infty]$ uma função diferenciável com relação a primeira variável. Definimos

$$
\tilde{d}_{i}(x, y) \stackrel{\text { def }}{=} d_{i}\left(x_{i}, y_{i}\right)+\frac{v}{2}\left(x_{i}-y_{i}\right)^{2} \neq
$$

Se $\widetilde{d}_{i}$ obedece às condições 4.1.1(a)-4.1.1(c) ${ }^{4}$ então chamaremos $\widetilde{d}_{i}$ de uma regula-

\footnotetext{
${ }^{1}$ Definido no Capítulo 1, Equação 1.4.

${ }^{2}$ Seja $\left\{x^{k}\right\}$ um seqüência obtida pelo Algoritmo 4.2.1. Se $x^{k} \rightarrow \mathcal{x}$, então, para cada $i=1, \ldots, n$, ou $\gamma_{i}^{k} \rightarrow \mathcal{K} 0$ ou existe um conjunto infinito $\mathcal{K}^{\prime} \subseteq \mathcal{K}$ tal que $x_{i}^{k-1} \rightarrow \mathcal{K}^{\prime} \bar{x}_{i}$.

${ }^{\ddagger} \mathrm{Em}[5]$, os autores consideram pesos diferentes para $d_{i}$ e para a norma euclidiana ao quadrado. Como os dois tipos de regularização coincidem a menos de uma multiplicação por uma constante, preferimos adotar peso 1 para $d_{i}$.

${ }^{4} \mathrm{~A}$ propriedade exigida na condição 4.1.1(d) será um conseqüência da Hipótese 5.1.2 apresentada a
} 
rização dupla.

\subsection{Uma Desigualdade Interessante}

Queremos provar que uma seqüência obtida pelo Algoritmo 4.2.1 baseado em regularizações duplas é Fejér monótona com relação ao conjunto de soluções da desigualdade variacional, de forma semelhante ao que acontece com o método proximal clássico. Para que isto seja possível, usaremos uma hipótese adicional que força o termo coercivo, $d_{i}$, a se manter "próximo" da norma euclidiana ao quadrado. Esta idéia é formalizada pela condição abaixo. ${ }^{5}$ Destacamos que, pela primeira vez, exigimos condições sobre o comportamento da derivada da regularização sem distinguir um lado com relação ao centro. Por exemplo, no caso $B=\mathbb{R}_{++}$, a Hipótese 4.1.1 limitava o comportamento de $d_{i}^{\prime}\left(x_{i}, y_{i}\right)$ somente se $y_{i}<x_{i}$, já a condição abaixo deve valer também se $x_{i}<y_{i}$.

Hipótese 5.1.2. Para cada $i=1, \ldots, n$, seja $d_{i}: \mathbb{R} \times\left(a_{i}, b_{i}\right) \rightarrow \mathbb{R}$ e $x, y \in\left(a_{i}, b_{i}\right)$, temos:

1. Caso $a_{i}$ e $b_{i}$ sejam finitos, então

$$
\frac{(x-y)\left(y-a_{i}\right)}{x-a_{i}} \leq d_{i}^{\prime}(x, y) \leq \frac{(x-y)\left(b_{i}-y\right)}{b_{i}-x} .
$$

2. Se o intervalo $\left(a_{i}, b_{i}\right)$ for ilimitado, tomando o respectivo limite nas desigualdades acima, obtemos:

(a) Apenas $a_{i}$ é finito $\left(b_{i}=+\infty\right)$.

$$
\frac{(x-y)\left(y-a_{i}\right)}{x-a_{i}} \leq d_{i}^{\prime}(x, y) \leq x-y .
$$

(b) Apenas $b_{i}$ é finito $\left(a_{i}=-\infty\right)$.

$$
x-y \leq d_{i}^{\prime}(x, y) \leq \frac{(x-y)\left(b_{i}-y\right)}{b_{i}-x}
$$

(c) $\left(a_{i}, b_{i}\right)=\mathbb{R}$.

$$
d_{i}^{\prime}(x, y)=x-y
$$

Usando esta condição, podemos demonstrar uma desigualdade fundamental para análise de nosso método proximal:

seguir. Desta forma, não há necessidade dela ser verificada. Isto é demonstrado no Lema 5.2.4.

${ }^{5}$ Esta condição é uma extensão da definição da classe de regularizações $\phi_{2}$, apresentada em [5]. Ver Seção 5.3 a seguir. 


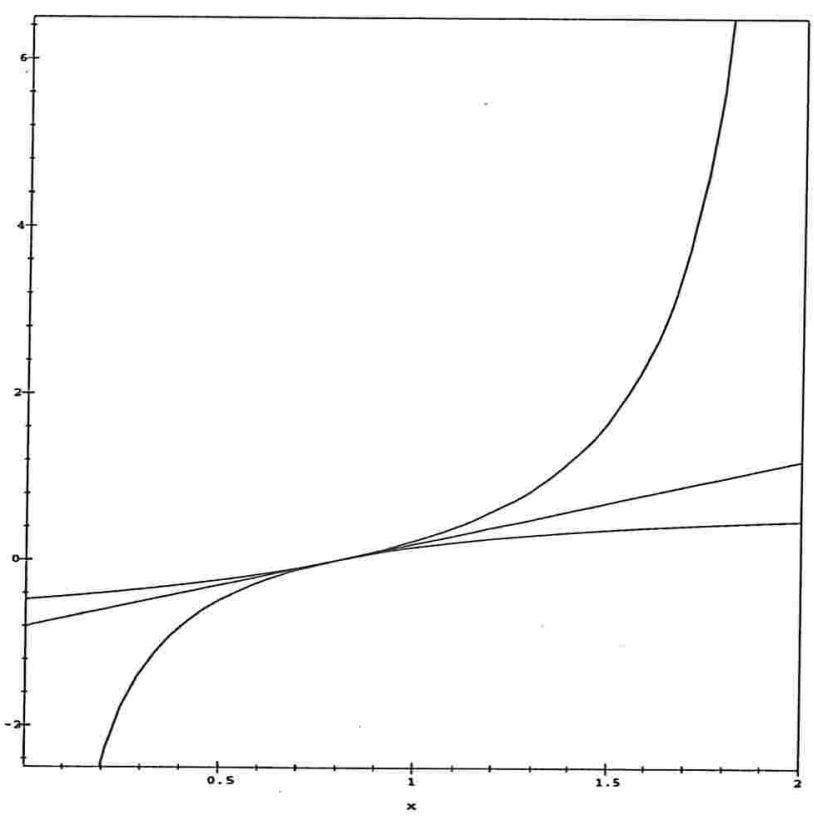

Figura 5.1: Limites para as derivadas de $d_{i}$ no caso do intervalo $\left(a_{i}, b_{i}\right)=(0,2)$. Observamos que a derivada da regularização clássica mantem-se entre os dois limites.

Lema 5.1.3. Se valer a Hipótese 5.1.2, então para todo $i=1, \ldots, n$ e $z \in\left[a_{i}, b_{i}\right] \cap$ $\mathbb{R}, x, y \in(a, b)$, temos:

$$
(z-x) d_{i}^{\prime}(x, y) \leq(z-y)(x-y)
$$

Demonstração. A desigualdade é trivial caso $x=y, x=z$ ou $y=z$.

Vamos considerar que $a_{i}, b_{i} \in \mathbb{R}$. O caso de intervalos ilimitados, como ocorre na própria Hipótese 5.1.2, pode ser visto como limite do caso de intervalos limitados.

O restante da demonstração será dividida em quatro casos:

1. $x<y, x<z$

Se $z<y$, é imediato que $(z-x) d^{\prime}(x, y) \leq 0 \leq(z-y)(x-y)$. 
Se $z>y$, então

$$
\begin{aligned}
\left(b_{i}-z\right) x & \leq\left(b_{i}-z\right) y \Rightarrow \\
b_{i} x+y z & \leq b_{i} y+x z \Rightarrow \\
x y-b_{i} x-y z+b_{i} z & \geq x y-b_{i} y-x z+b_{i} z \Rightarrow \\
(z-x)\left(b_{i}-y\right) & \geq(z-y)\left(b_{i}-x\right) \Rightarrow \\
\frac{b_{i}-y}{b_{i}-x} & \geq \frac{z-y}{z-x} \Rightarrow \\
\frac{(x-y)\left(b_{i}-y\right)}{b_{i}-x} & \leq \frac{(x-y)(z-y)}{z-x} \stackrel{\text { Hip. 5.1.2 }}{\longrightarrow} \\
d_{i}^{\prime}(x, y) & \leq \frac{(x-y)(z-y)}{z-x} \Rightarrow \\
(z-x) d_{i}^{\prime}(x, y) & \leq(x-y)(z-y) .
\end{aligned}
$$

2. $x<y, x>z$

Seguindo uma seqüência de passos análogos as que nos levaram da desigualdade (5.3) à desigualdade (5.4), podemos concluir que

$$
\begin{aligned}
\left(z-a_{i}\right) x & \leq\left(z-a_{i}\right) y \Rightarrow \\
\frac{y-z}{x-z} & \geq \frac{y-a_{i}}{x-a_{i}} .
\end{aligned}
$$

Multiplicando-se esta última desigualdade por $x-y$ e aplicando a Hipótese 5.1.2, concluímos mais uma vez (5.2).

3. $x>y, x>z$

Como no primeiro caso, se $z>y$, temos $(z-x) d^{\prime}(x, y) \leq 0 \leq(z-y)(x-y)$.

Caso $z<y$, usamos as mesmas idéias presentes acima para concluir que

$$
\begin{aligned}
\left(z-a_{i}\right) x & \geq\left(z-a_{i}\right) y \Rightarrow \\
\frac{y-z}{x-z} & \leq \frac{y-a_{i}}{x-a_{i}} .
\end{aligned}
$$

Outra vez multiplicamos esta última por $x-y$ e usamos a Hipótese 5.1 .2 e obte$\operatorname{mos}(5.2)$.

4. $x>y, x<z$

Análogo aos casos anteriores. 


\subsection{Análise de Convergência}

Já dispomos de todas as ferramentas necessárias a prova de convergência da seguinte instância do Algoritmo 4.2.1:

\section{Algoritmo 5.2.1. Método Proximal com Regularizações Duplas para Desigualdades Variacionais}

Seja $\widetilde{\alpha}$ um real positivo. Sejam $\tilde{d}_{i}, i=1, \ldots, n$, regularizações duplas obedecendo à Hipótese 5.1.2. Defina

$$
D(x, y) \stackrel{\text { def }}{=} \sum_{i=1}^{n} \tilde{d}_{i}\left(x_{i}, y_{i}\right)=\sum_{i=1}^{n} d_{i}\left(x_{i}, y_{i}\right)+\frac{\nu}{2}\left(x_{i}-y_{i}\right)^{2} .
$$

1. Inicialização: Comece $\operatorname{com} x^{1} \in \operatorname{int} B$;

2. Iteração: Escolha um real $0<\alpha_{k} \leq \widetilde{\alpha}$, e encontre $x^{k+1} \in \operatorname{int} B \cap \operatorname{dom} T$ tal que

$$
0 \in \mathrm{T}\left(x^{k+1}\right)+\alpha_{k} \nabla_{1} \mathrm{D}\left(x^{k+1}, x^{k}\right) . \|
$$

Se $x^{k+1}=x^{k}$, pare.

Para provar a convergência da seqüência $\left\{x^{k}\right\}$ a uma solução do problema da desigualdade variacional, vamos mostrar que esta seqüência se aproxima do conjunto de soluções. Começamos pelo seguinte lema:

Lema 5.2.2. Seja D como no Algoritmo 5.2.1. Então, para todo $z \in \mathrm{B}$ e $\mathrm{x}, \mathrm{y} \in \mathrm{int} \mathrm{B}$,

$$
\left\langle z-x, \nabla_{1} \mathrm{D}(x, y)\right\rangle \leq \frac{v+1}{2}\left(\|z-y\|^{2}-\|z-x\|^{2}\right)-\frac{v-1}{2}\|x-y\|^{2} .
$$

Demonstração. Para cada $i=1, \ldots, n$, temos:

$$
\begin{aligned}
\left(z_{i}-x_{i}\right) \nabla_{1} D(x, y)_{i} & =\left(z_{i}-x_{i}\right)\left(d_{i}^{\prime}\left(x_{i}, y_{i}\right)+v\left(x_{i}-y_{i}\right)\right) \\
& \leq\left(z_{i}-y_{i}\right)\left(x_{i}-y_{i}\right)+v\left(z_{i}-x_{i}\right)\left(x_{i}-y_{i}\right) . \quad \text { [Lema 5.1.3] }
\end{aligned}
$$

Somando todos os termos obtemos:

$$
\left\langle z-x, \nabla_{1} \mathrm{D}(x, y)\right\rangle \leq\langle z-y, x-y\rangle+v\langle z-x, x-y\rangle .
$$

\footnotetext{
"Devemos destacar que a exigência de solução exata dos sub-problemas do Algoritmo 5.2.1 é feita por simplicidade de exposição. Para adoção de erros na resolução dos sub-problemas, poder-se-ia utilizar uma fórmula semelhante ao critério de aceitação proposto por Eckstein em [20] e retomado por Silva, Eckstein e Humes na última seção de [42].
} 
Lembrando que,

$$
\begin{aligned}
& \langle z-y, x-y\rangle=\frac{\|z-y\|^{2}-\|z-x\|^{2}+\|x-y\|^{2}}{2} \\
& \langle z-x, x-y\rangle=\frac{\|z-y\|^{2}-\|z-x\|^{2}-\|x-y\|^{2}}{2},
\end{aligned}
$$

podemos concluir que

$$
\left\langle z-x, \nabla_{1} D(x, y)\right\rangle \leq \frac{v+1}{2}\left(\|z-y\|^{2}-\|z-x\|^{2}\right)-\frac{v-1}{2}\|x-y\|^{2} .
$$

A propriedade de aproximação e a validade da Hipótese 4.2.2, mencionadas na introdução, tornam-se, então, uma conseqüência imediata:

Lema 5.2.3. Seja $\left\{x^{k}\right\}$ uma seqüência obtida pelo Algoritmo 5.2.1. A seqüência $\left\{x^{k}\right\}$ é Fejér monótona com relação ao conjunto de soluçôes da desigualdade variacional (1.4). Ainda, caso o conjunto de soluções de (1.4) seja não vazio, então $x^{\mathrm{k}+1}-x^{\mathrm{k}} \rightarrow 0$, portanto, a Hipótese 4.2.2 é válida.

Demonstração. Seja $z \in\left(T+N_{B}\right)^{-1}(0)$. Como em toda iteração $x^{k} \in \operatorname{int} B$ e os passos são realizados com exatidão, obtemos $-\alpha_{k} \nabla_{1} \mathrm{D}\left(x^{k+1}, x^{k}\right) \in\left(T+N_{B}\right)\left(x^{k+1}\right)$. Usando a monotonicidade de $T+N_{B}$ e o Lema 5.2.2, concluímos que:

$$
\begin{aligned}
0 & \leq\left\langle z-x^{k+1}, \alpha_{k}^{-1} \nabla_{1} \mathrm{D}\left(x^{k+1}, x^{k}\right)\right\rangle \\
& \leq \frac{v+1}{2 \alpha_{k}}\left(\left\|z-x^{k}\right\|^{2}-\left\|z-x^{k+1}\right\|^{2}\right)-\frac{v-1}{2 \alpha_{k}}\left\|x^{k+1}-x^{k}\right\|^{2} .
\end{aligned}
$$

Portanto,

$$
\left\|z-x^{k+1}\right\| \leq\left\|z-x^{k}\right\|,
$$

$\mathrm{O}$ que implica a Fejér monotonicidade de $\left\{x^{k}\right\}$. Além disto, como $v>1$,

$$
\left\|x^{k+1}-x^{k}\right\|^{2} \leq \frac{v+1}{v-1}\left(\left\|z-x^{k}\right\|^{2}-\left\|z-x^{k+1}\right\|^{2}\right) \text {. }
$$

Como a Fejér monotonicidade assegura que $\left\|z-x^{k}\right\|$ é convergente para qualquer $z \in$ $\left(T+N_{B}\right)^{-1}(0)$, se $\left(T+N_{B}\right)^{-1}(0)$ for não-vazio, teremos $\left\|x^{k+1}-x^{k}\right\| \rightarrow 0$.

Finalmente, para que possamos usar o teorema de convergência do Algoritmo 4.2.1, basta provar que as regularizações duplas, $\widetilde{\mathrm{d}}_{i}, i=1, \ldots, n$, obedecem à Hipótese 4.1.1(d). ${ }^{7}$ Faremos isto a seguir.

\footnotetext{
${ }^{7}$ Existem $L, \epsilon>0$ tais que, sempre que $-\infty<a_{i}<y_{i} \leq x_{i}<a_{i}+\epsilon$ ou $b_{i}-\epsilon<x_{i} \leq y_{i}<b_{i}<+\infty$ temos que $\left|d_{i}^{\prime}\left(x_{i}, y_{i}\right)\right| \leq L\left|x_{i}-y_{i}\right|$.
} 
Lema 5.2.4. Seja $\epsilon=\min _{i=1, \ldots, n}\left\{\left(b_{i}-a_{i}\right) / 2\right\}$. Se $-\infty<a_{i}<y_{i} \leq x_{i}<a_{i}+\epsilon$ ou $\mathrm{b}_{i}-\epsilon<x_{i} \leq y_{i}<b_{i}<+\infty$, então $\left|d_{i}^{\prime}\left(x_{i}, y_{i}\right)\right| \leq 2\left|x_{i}-y_{i}\right|$. Conseqüentemente $\left|\tilde{\mathrm{d}}_{i}^{\prime}\left(x_{i}, y_{i}\right)\right| \leq(2+v)\left|x_{i}-y_{i}\right|$.

Demonstração. Se $-\infty<a_{i}<y_{i} \leq x_{i}<a_{i}+\epsilon$ e $b_{i}=\infty$ a tese segue facilmente da Hipótese 5.1.2. Caso $b_{i} \in \mathbb{R}$ temos

$$
\begin{aligned}
\left|d_{i}^{\prime}\left(x_{i}, y_{i}\right)\right| & =d_{i}^{\prime}\left(x_{i}, y_{i}\right) \\
& \leq \frac{\left(x_{i}-y_{i}\right)\left(b_{i}-y_{i}\right)}{b_{i}-x_{i}} \\
& =\frac{\left|x_{i}-y_{i}\right|\left(b_{i}-y_{i}\right)}{b_{i}-x_{i}} \\
& \leq \frac{\left|x_{i}-y_{i}\right|\left(b_{i}-a_{i}\right)}{\left(b_{i}-a_{i}-\epsilon\right)} \\
& \leq 2\left|x_{i}-y_{i}\right| .
\end{aligned}
$$

$\mathrm{O}$ caso $\mathrm{b}_{\mathrm{i}}-\epsilon<\mathrm{x}_{\mathrm{i}} \leq \mathrm{y}_{\mathrm{i}}<\mathrm{b}_{\mathrm{i}}<+\infty$ é análogo, usando a outra desigualdade dada pela Hipótese 5.1.2.

Teorema 5.2.5. Se o conjunto de soluções da desigualdade variacional for não-vazio, então uma seqüência obtida pelo Algoritmo 5.2.1 converge a uma solução.

Demonstração. Como assumimos que as regularizações $\widetilde{\mathrm{d}}_{i}, i=1, \ldots, n$, obedecem às Condições 4.1.1(a)-4.1.1(c), podemos usar o Lema 5.2.4 para ver que a Hipótese 4.1.1 está inteiramente satisfeita. Além disto, o Lema 5.2.3 garante que a Hipótese 4.2.2 também vale. Portanto, podemos usar o Teorema 4.2.6 para assegurar que todo ponto de acumulação de $\left\{x^{k}\right\}$ é uma solução da desigualdade variacional.

Deste modo, a convergência de $\left\{x^{k}\right\}$ a uma solução é uma conseqüência de sua Fejér monotonicidade.

\subsection{Exemplos}

Nesta seção, vamos apresentar alguns exemplos de regularizações coercivas que obedecem à Hipótese 5.1.2, e, portanto, podem ser usadas para construir uma dupla regularização que garanta a convergência da seqüência proximal a uma solução do problema da desigualdade variacional.

Vamos nos concentrar no caso $(a, b)=\mathbb{R}_{++}$. A partir de uma regularização deste tipo é fácil, através de translações e mudanças de sinal, obter uma regularização para intervalos com extremos quaisquer. Por exemplo temos: 
Lema 5.3.1. Seja d: $\mathbb{R} \times \mathbb{R}_{++} \rightarrow(-\infty, \infty]$ uma função que obedece à Hipótese 5.1.2. Então, dados $\mathrm{a}, \mathrm{b} \in \mathbb{R}, \mathrm{a}<\mathrm{b}$ e $\zeta \in(0,1)$, temos que

$$
\widehat{d}(x, y) \stackrel{\text { def }}{=} \zeta d(x-a, y-a)+(1-\zeta) d(b-x, b-y)
$$

obedece a esta mesma condição para o intervalo $(\mathrm{a}, \mathrm{b})$.

Demonstração. Dados , $\mathrm{y} \in(\mathrm{a}, \mathrm{b})$, Usamos a Hipótese 5.1.2 para d e obtemos:

$$
\frac{\zeta(x-a-y+a)(y-a)}{x-a} \leq \zeta d^{\prime}(x-a, y-a) \leq \zeta(x-a-y+a),
$$

e também

$$
\frac{(1-\zeta)(b-x-b+y)(b-y)}{b-x} \leq(1-\zeta) d^{\prime}(b-x, b-y) \leq(1-\zeta)(b-x-b+y)
$$

Multiplicando a segunda desigualdade por -1 e somando temos:

$$
\begin{aligned}
\frac{\zeta(x-y)(y-a)}{x-a}+(1-\zeta)(x-y) & \leq \zeta d^{\prime}(x-a, y-a)-(1-\zeta) d^{\prime}(b-x, b-y) \\
& \leq \frac{(1-\zeta)(x-y)(b-y)}{b-x}+\zeta(x-y) .
\end{aligned}
$$

Por outro lado, lembrando que, $x, y \in(a, b)$, é fácil ver que

$$
\begin{aligned}
& \frac{(x-y)(y-a)}{x-a} \leq x-y \\
& x-y \leq \frac{(x-y)(b-y)}{b-x}
\end{aligned}
$$

Usando a definição de $\hat{d}$, segue que para $x, y \in(a, b)$

$$
\frac{(x-y)(y-a)}{x-a} \leq \hat{d}^{\prime}(x, y) \leq \frac{(x-y)(b-y)}{b-x} \text {. }
$$

\section{$\varphi$-divergências}

Como destacamos na introdução, os resultados deste capítulo podem ser vistos como uma extensão de idéias introduzidas em [5, 4]. Nestes artigos, os autores consideram termos coercivos obtidos pela técnica de mudança de escala aplicada a $\varphi$-divergências ${ }^{8}$

\footnotetext{
${ }^{8}$ Ver Seção 4.1, na qual mostramos que a mudança de escala recupera a regra de formação de regularizações coercivas usada por estes autores.
} 
e adicionam a norma euclidiana ao quadrado obtendo um regularização dupla para o ortante positivo.

Vamos provar que a Hipótese 5.1.2 nada mais é do que uma extensão da definição da classe de regularizações $\phi_{2}$ de [5]. Neste trabalho, os termos coercivos têm a forma:

$$
d_{i}(x, y)=y^{2} \varphi\left(\frac{x}{y}\right)
$$

para alguma $\varphi: \mathbb{R}_{+} \rightarrow \mathbb{R}$ obedecendo à Hipótese 4.1.4. Neste caso a Hipótese 5.1.2 torna-se:

$$
\forall x, y \in \mathbb{R}_{++}, \frac{(x-y) y}{x} \leq y \varphi^{\prime}\left(\frac{x}{y}\right) \leq x-y .
$$

Fazendo a troca de variáveis $t=\frac{x}{y}$, esta desigualdade mostra-se equivalente a

$$
\forall t>0,(1-1 / t) \leq \varphi^{\prime}(t) \leq(t-1)
$$

que é usada para definir a classe $\phi_{2}$.

Por fim, Auslender, Teboulle e Ben-Tiba apresentam os seguintes exemplos de funções que obedecem a esta última desigualdade:

1. $\varphi(t)=t \log (t)-t+1$;

2. $\varphi(t)=2(\sqrt{t}-1)^{2} ; \ddagger \ddagger$

3. $\varphi(t)=-\log (t)+t-1 . \ddagger$

Entre estes exemplos destacamos $\varphi(t)=-\log (t)+t-1$, que dá origem à regularização logarítmica-quadrática, primeira regularização dupla estudada na literatura [4]. Outra característica interessante é que, para esta $\varphi$,

$$
d_{\varphi}^{\prime}(x, y)=\frac{(x-y) y}{x}
$$

Ou seja, a derivada de sua distância coincide com o limite inferior da Hipótese 5.1.2.

\section{Distâncias de Bregman}

Vamos apresentar funções de Bregman que gerem distâncias obedecendo à Hipótese 5.1.2 após a mudança de escala. ${ }^{10}$ Desta forma, seremos capazes de resolver problemas variacionais sem a necessidade de hipóteses de para-monotonicidade comuns na literatura

\footnotetext{
${ }^{\ddagger \ddagger}$ Como adicionamos o termo quadrático ao termo coercivo para obter $\tilde{\mathrm{d}}$, temos rge $\widetilde{\mathrm{d}}_{\mathrm{i}}^{\prime}(\cdot, y)=\mathbb{R}$ e a dificuldade associada ao uso destas regularizações, comentada na Seção 4.1, desaparece.

${ }^{10}$ Ver Seção 4.1.
} 
que trata deste tipo de regularização [27, 44, 35]. Observamos que [42, Seção 4] obtem resultados semelhantes, porém fazendo uso de uma versão mais forte da mudança de escala, na qual, para $x, y \in B$ :

$$
\begin{gathered}
\alpha=\max _{i=1, \ldots, n}\left\{h_{i}^{\prime \prime}\left(y_{i}\right)\right\} \\
D(x, y)=\sum_{i=1}^{n} \frac{h_{i}\left(x_{i}\right)-h_{i}\left(y_{i}\right)-h_{i}\left(y_{i}\right)\left(x_{i}-y_{i}\right)}{\alpha} .
\end{gathered}
$$

Destacamos que neste caso o fator de escala vai para zero mesmo que a respectiva coordenada não se aproxime dos extremos da caixa B, o que não ocorre com as regularizações duplas.

Antes dos exemplos, vamos apresentar um pequeno lema que pode simplificar bastante a verificação da Hipótese 5.1.2:

Lema 5.3.2. Seja $h: \mathbb{R} \rightarrow(-\infty, \infty]$, int dom $h=\mathbb{R}_{++}$. Se, para $\mathrm{x} \in \mathbb{R}_{++}, \mathrm{h}^{\prime \prime}(\mathrm{x})$ for não-crescente e $\mathrm{x}^{2} \mathrm{~h}^{\prime \prime}(\mathrm{x})$ for não-decrescente, então a distância generalizada, dada por

$$
d(x, y)=\frac{h(x)-h(y)-h^{\prime}(y)(x-y)}{h^{\prime \prime}(y)},
$$

obedece à Hipótese 5.1.2, para $(\mathrm{a}, \mathrm{b})=\mathbb{R}_{++}$.

Demonstração. Para $(\mathrm{a}, \mathrm{b})=\mathbb{R}_{++}$, temos que o limite inferior de $\mathrm{d}^{\prime}(\mathrm{x}, \mathrm{y})$, dado pela Hipótese 5.1.2, reduz-se a $h^{\prime \prime}(y)(x-y) y \leq\left(h^{\prime}(x)-h^{\prime}(y)\right) x$. Para provar que esta desigualdade é válida, consideraremos dois casos:

1. Se $0<x<y$, temos:

$$
\begin{array}{rlr}
h^{\prime}(y)-h^{\prime}(x) & =\int_{x}^{y} h^{\prime \prime}(z) d z & \\
& \leq \int_{x}^{y} \frac{y^{2} h^{\prime \prime}(y)}{z^{2}} d z \quad\left[x^{2} h^{\prime \prime}(x) \text { não-decr. }\right] \\
& =h^{\prime \prime}(y)\left(-\frac{1}{y}+\frac{1}{x}\right) y^{2} & \\
& =h^{\prime \prime}(y) \frac{y-x}{x y} y^{2} \\
& =h^{\prime \prime}(y)(y-x) \frac{y}{x} .
\end{array}
$$


2. Se $0<y<x$, seguimos a idéia do ítem anterior para concluir que

$$
\begin{array}{rlrl}
h^{\prime}(x)-h^{\prime}(y) & =\int_{y}^{x} h^{\prime \prime}(z) d z & \\
& \geq \int_{y}^{x} \frac{y^{2}}{z^{2}} h^{\prime \prime}(y) d z & {\left[x^{2} h^{\prime \prime}(x) \text { não-decr. }\right]} \\
& =h^{\prime \prime}(y)(x-y) \frac{y}{x} .
\end{array}
$$

Já o limite superior torna-se: $h^{\prime}(x)-h^{\prime}(y) \leq h^{\prime \prime}(y)(x-y)$. Para verificar que este limite vale, consideramos mais uma vez dois casos:

1. Se $0<x_{i}<y_{i}$.

$$
\begin{aligned}
h^{\prime}(y)-h^{\prime}(x) & =\int_{x}^{y} h^{\prime \prime}(z) d z & \\
& \geq \int_{x}^{y} h^{\prime \prime}(y) d z & {\left[h^{\prime \prime}(x) \text { não-cresc. }\right] } \\
& =h^{\prime \prime}(y)(y-x) . &
\end{aligned}
$$

2. O caso $0<y<x$ é análogo ao caso anterior.

Alguns exemplos de funções de Bregman que podemos usar para obter regularizações duplas são:

1. $h(x)=\operatorname{dilog}\left(e^{x}\right)+x \log \left(e^{x}-1\right)$. Neste caso,

$$
h^{\prime \prime}(x)=\frac{e^{x}}{e^{x}-1}
$$

Claramente, $h^{\prime \prime}(x)$ é não-crescente.

Por outro lado, para provarmos que $x^{2} h^{\prime \prime}(x)$ é não-decrescente, vamos provar que sua derivada é sempre positiva. Esta derivada é

$$
\frac{e^{x} x\left(2 e^{x}-x-2\right)}{\left(e^{x}-1\right)^{2}}
$$

Seu sinal é definido pelo sinal de $2 e^{x}-x-2$, que vale 0 , se $x=0$, e é estritamente crescente. Logo o sinal da derivada de $x^{2} h^{\prime \prime}(x)$ é sempre positivo.

Portanto esta função gera uma distância de Bregman que obedece à Hipótese 5.1.2. 
2. $h(x)=x^{\alpha}-x^{\beta}, \alpha \geq 1, \beta \in(0,1)$. Aqui,

$$
\begin{aligned}
& h^{\prime \prime}(x)=\alpha(\alpha-1) x^{\alpha-2}+\beta(1-\beta) x^{\beta-2} . \\
& x^{2} h^{\prime \prime}(x)=\alpha(\alpha-1) x^{\alpha}+\beta(1-\beta) x^{\beta} .
\end{aligned}
$$

Notamos que $x^{2} h^{\prime \prime}(x)$ é sempre não-decrescente em $\mathbb{R}_{++}$e $h^{\prime \prime}(x)$ é não-crescente se, e somente se, $\alpha \leq 2$.

Logo, a distância de Bregman obtida a partir de $h$ obedecerá à Hipótese 5.1.2 se, e somente se, $\alpha \in[1,2]$ e $\beta \in(0,1)$.

\subsection{Penalidades e Métodos de Multiplicadores}

Nesta seção, vamos caracterizar penalidades geradas a partir de regularizações duplas usando a Hipótese 5.1.2. Em seguida discutiremos brevemente sua relação com um artigo de Chen e Mangasarian [17] que pode nos ajudar a encontrar novas penalidades.

Lema 5.4.1. Seja $v>1$. Seja $\mathrm{P}: \mathbb{R} \times \mathbb{R}_{++} \rightarrow \mathbb{R}$, denotemos por $\mathrm{P}^{\prime}(u, \lambda)$ a derivada de $\mathrm{P}$ com respeito à primeira variável. Se $\mathrm{P}^{\prime}(\cdot, \lambda)$ for estritamente crescente e estritamente positiva e, além disto,

$$
\frac{u}{v+1}+\lambda \leq P^{\prime}(u, \lambda) \leq \frac{u+(v-1) \lambda+\sqrt{(u+(v-1) \lambda)^{2}+4 v \lambda^{2}}}{2 v}
$$

então existe uma regularização dupla, $\widetilde{\mathrm{d}}$, que obedece à Hipótese 5.1.2 e tal que

$$
P(u, \lambda)=(\tilde{d}(\cdot, \lambda))^{*}(u)
$$

Demonstração. Dado $\lambda \in \mathbb{R}_{++}$, a derivada de $\mathrm{P}(\cdot, \lambda)$ é estritamente crescente, logo esta função é estritamente convexa. Chamemos de $\tilde{d}(\cdot, \lambda)$ sua função convexo conjugada.

Sabemos que:

1. Para todo $\lambda \in \mathbb{R}_{++}, \widetilde{\mathrm{d}}(\cdot, \lambda)$ é estritamente convexa, pois $\mathrm{P}(\cdot, \lambda)$ é diferenciável.

2. Para todo $\lambda \in \mathbb{R}_{++}, \widetilde{d}(\cdot, \lambda)$ é diferenciável, pois $P(\cdot, \lambda)$ é estritamente convexa. Além disto, $\tilde{\mathrm{d}}^{\prime}(\cdot, \lambda)=\left(\mathrm{P}^{\prime}\right)^{-1}(\cdot, \lambda)$.

3. Usando os limites sobre $\mathrm{P}^{\prime}(\cdot, \lambda)$, concluímos que $\mathrm{P}^{\prime}(0, \lambda)=\lambda$. Portanto $\tilde{\mathrm{d}}^{\prime}(\lambda, \lambda)=$ 0 , ou seja, $\widetilde{d}(\cdot, \lambda)$ atinge mínimo em $\lambda$. 
4. Como $\tilde{\mathrm{d}}(\cdot, \lambda)$ é a convexo conjugada de uma penalidade, temos dom $\widetilde{\mathrm{d}} \subset \mathbb{R}_{+}$ (Lema 2.2.2). Ainda, uma vez que $\operatorname{dom} \mathrm{P}^{\prime}(\cdot, \lambda)=\mathbb{R}$, então rge $\tilde{\mathrm{d}}^{\prime}(\cdot, \lambda)=\mathbb{R}$.

Definindo $d(\gamma, \lambda) \stackrel{\text { def }}{=} \widetilde{d}(\gamma, \lambda)-\frac{v}{2}\|\gamma-\lambda\|^{2}$, as propriedades acima asseguram que $\widetilde{d}$ é uma regularização dupla caso seja verdade que para todo $\lambda \in \mathbb{R}_{++}$, se $\left\{\left(\gamma^{k}, \lambda^{k}\right)\right\}$ é uma seqüência em $\mathbb{R}_{++}$convergindo para $(\lambda, \lambda)$, então $\tilde{\mathrm{d}}^{\prime}\left(\gamma^{k}, \lambda^{k}\right) \rightarrow 0$. Mas, isto é uma conseqüência da Hipótese 5.1.2 aplicada à d e, portanto, devemos apenas provar sua validade. Faremos isto em duas etapas:

1. Começamos por

$$
\begin{aligned}
\frac{u}{v+1}+\lambda & \leq \mathrm{P}^{\prime}(u, \lambda) \Longleftrightarrow \\
\left(\mathrm{P}^{\prime}\right)^{-1}\left(\frac{u}{v+1}+\lambda, \lambda\right) & \leq u \\
\widetilde{\mathrm{d}}^{\prime}\left(\frac{u}{v+1}+\lambda, \lambda\right) & \leq u .
\end{aligned}
$$

Fazendo a mudança de variáveis $\gamma \stackrel{\text { def }}{=} \frac{u}{v+1}+\lambda$ e lembrando a definição de $d$, obtemos

$$
\forall \gamma \in \mathbb{R}_{++}, \mathrm{d}^{\prime}(\gamma, \lambda) \leq(\gamma-\lambda) .
$$

2. Por outro lado,

$$
\begin{aligned}
P^{\prime}(u, \lambda) & \leq \frac{u+(v-1) \lambda+\sqrt{(u+(v-1) \lambda)^{2}+4 v \lambda^{2}}}{2 v} \Longleftrightarrow \\
u & \leq \tilde{d}^{\prime}\left(\frac{u+(v-1) \lambda+\sqrt{(u+(v-1) \lambda)^{2}+4 v \lambda^{2}}}{2 v}, \lambda\right)
\end{aligned}
$$

De novo, trocamos variáveis: $\gamma \stackrel{\text { def }}{=} \frac{u+(\nu-1) \lambda+\sqrt{(u+(v-1) \lambda)^{2}+4 v \lambda^{2}}}{2 v}$

$$
\forall \gamma \in \mathbb{R}_{++}, \frac{(\gamma-\lambda) \lambda}{\gamma} \leq \mathrm{d}^{\prime}(\gamma, \lambda)
$$

A demonstração está completa. Destacamos ainda que, como provamos que a desigualdade (5.8) é equivalente a Hipótese 5.1.2, demonstramos, implicitamente, que (5.8) é coerente (o limite inferior apresentado é sempre menor do que o limite superior, como observamos na Figura 5.4). 


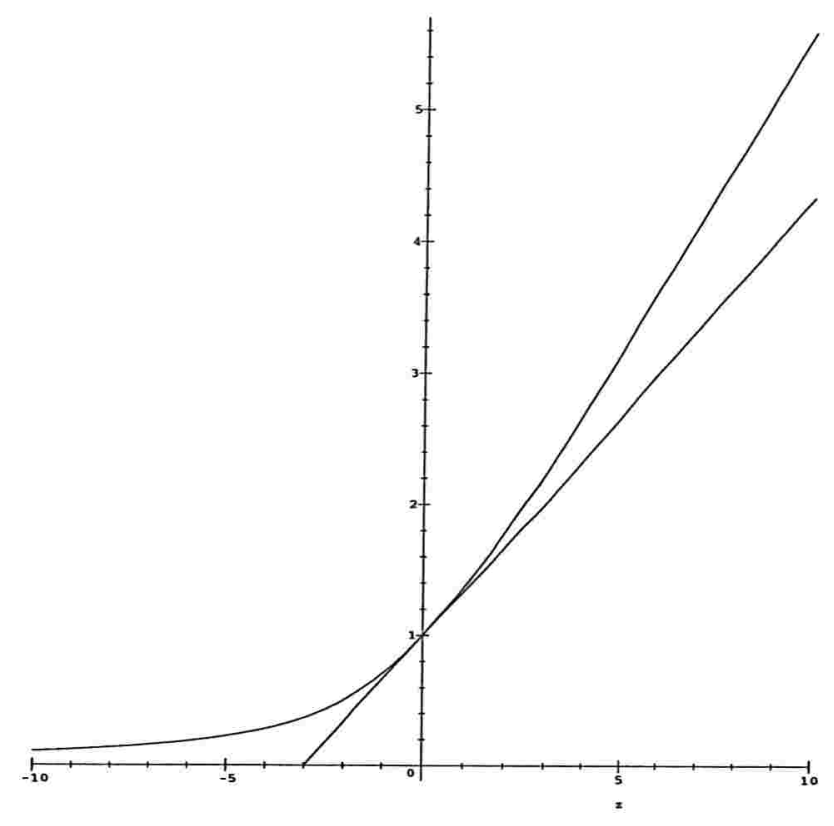

Figura 5.2: Os limites para derivada de uma penalidade no caso $\lambda=1$. acima,

Observamos que o limite superior para a derivada de uma penalidade dado no lema

$$
\frac{u+(v-1) \lambda+\sqrt{(u+(v-1) \lambda)^{2}+4 v \lambda^{2}}}{2 v},
$$

coincide com a derivada da penalidade obtida a partir da regularização logarítmicaquadrática. ${ }^{11}$

Outro fato interessante, e que não foi destacado por Auslender, Teboulle e Ben-Tiba em $[4,5]$, é que esta penalidade já havia sido apresentada, implicitamente, por Chen e Mangasarian no contexto de métodos de penalização puros [17]. Neste artigo, os autores sugerem o uso de uma família de aproximações da função $\max (u, 0)$ para a solução de problemas variacionais. Entre elas está a Chen-Harker-Kanzow-Smale Plus Function, que é definida a partir de um parâmetro de penalização, $\beta$, como:

$$
P^{\prime}(u, \beta) \stackrel{\text { def }}{=} \frac{u+\sqrt{u^{2}+4 \beta^{2}}}{2} .
$$

Recuperamos a derivada da penalidade baseada na regularização logarítmica-quadrática através de $P^{\prime}(u+(v-1) \lambda, \sqrt{v} \lambda)$.

\footnotetext{
${ }^{11}$ Assim como o limite inferior dado pela Hipótese 5.1.2 coincide com a derivada desta regularização. Ver Seção 5.3 .
} 
Acreditamos que um estudo cuidadoso da família de aproximações da função máximo sugerida em [17] pode levar a outras penalidades associadas a regularizações duplas. Em particular, seria interessante obter uma penalidade baseada na neural networks smooth plus function, já que esta função apresentou os melhores resultados numéricos.

Por fim, devemos destacar que as penalidades obtidas a partir de regularizações duplas podem ser usadas para obter diferentes versões de métodos de multiplicadores para a solução de problemas variacionais com restrições convexas gerais, descobertos recentemente por Auslender e Teboulle [3] e Pennanen [32]. 


\section{Resolução Aproximada em Métodos de Multiplicadores}

Neste capítulo, apresentaremos novos critérios de aceitação de soluções aproximadas dos sub-problemas irrestritos que compõem os passos iterativos de um método de multiplicadores. Destacamos que vamos restringir nossa análise a problemas de programação convexa (1.2) cujas funções são diferenciáveis em todo o $\mathbb{R}^{n}$. Assumimos ainda que vale uma condição de qualificação, como a condição de Slater ou a condição estrita ${ }^{1}$, e, portanto, os problemas primal e dual possuem mesmo valor ótimo.

Analisaremos dois métodos. Primeiro, o Algoritmo 4.3.5, apresentado no Capítulo 4. Neste caso, vamos obter um critério semelhante aos critérios de [39, 5], cuja versão implementável depende de hipóteses extras sobre o problema primal. Em seguida, discutiremos o uso do método híbrido de Solodov e Svaiter, Algoritmo 2.1.7, para a busca de um ponto de sela do lagrangiano associado ao primal. A respectiva condição para aceitação de soluções aproximadas será bastante permissiva e não dependerá de hipóteses adicionais sobre o problema que desejamos resolver.

\subsection{Métodos de Multiplicadores com Distâncias Co- ercivas}

Sejam $\mathrm{d}_{\mathrm{i}}: \mathbb{R} \times \mathbb{R} \rightarrow(-\infty, \infty], i=1, \ldots, \mathrm{m}$ distâncias coercivas generalizadas obedecendo à Hipótese 4.1.1 para $\left(\mathrm{a}_{i}, \mathrm{~b}_{i}\right)=(0,+\infty)$. Como desejamos usar o Algoritmo 4.3.5, assumimos que existe um ponto dual viável e estritamente positivo.

\footnotetext{
${ }^{1}$ Estes e outros exemplos de condições de qualificação são apresentados por Mangasarian em [29, Capítulos 5 e 7].
} 
Uma conseqüência, fundamental em nossa análise, da coercitividade de $d_{i}$ em $\mathbb{R}_{++}$ é que, fixo $\lambda_{i}>0$, as penalidades associadas a estas regularizações se reduzem à função convexo conjugada de $d_{i}$ com relação à sua primeira variável:

$$
P_{i}\left(u_{i}, \lambda_{i}\right) \stackrel{\text { def }}{=}\left(d_{i}\left(\cdot, \lambda_{i}\right)+\delta_{\mathbb{R}_{+}}(\cdot)\right)^{*}\left(u_{i}\right), i=1, \ldots, m .
$$

Desejamos estudar seqüências do tipo:

$$
\begin{aligned}
0 & <\alpha_{i}^{k} \leq \widetilde{\alpha}, i=1, \ldots, m ; \\
x^{k+1} & \approx \underset{x \in \mathbb{R}^{n}}{\operatorname{Argmin}}\left\{f(x)+\sum_{i=1}^{m} \alpha_{i}^{k} P_{i}\left(\frac{g_{i}(x)}{\alpha_{i}^{k}}, \lambda_{i}^{k}\right)\right\} ; \\
\lambda_{i}^{k+1} & =\frac{\partial P_{i}}{\partial u_{i}}\left(\frac{g_{i}\left(x^{k+1}\right)}{\alpha_{i}^{k}}, \lambda_{i}^{k}\right), i=1, \ldots, m .
\end{aligned}
$$

Que nada maís são do que o Algoritmo 4.3.5 com resolução aproximada do sub-problema de minimização. Lembrando que Algoritmo 4.3.5 é uma especialização do Algoritmo 4.3.1 aplicado ao problema de maximização da função dual, F. Podemos tentar usar o seu critério de aceitação, expresso pela Desigualdade (4.12), para decretar que um novo ponto primal pode ser aceito.

Seja $\sigma \in[0,1]$ e denotemos $D \stackrel{\text { def }}{=} \sum_{i=1}^{m} d_{i}$. (6.1) implica que a Equação (6.4) é equivalente a

$$
g_{i}\left(x^{k+1}\right)=\alpha_{i}^{k} d_{i}^{\prime}\left(\lambda_{i}^{k+1}, \lambda_{i}^{k}\right), i=1, \ldots, m .
$$

Portanto, a condição (4.12), reduz-se a

$$
\exists \gamma_{F} \in \partial F\left(\lambda^{k+1}\right),\left|\left(\gamma_{F}\right)_{i}-g_{i}\left(x^{k+1}\right)\right| \leq \sigma\left|g_{i}\left(x^{k+1}\right)\right|, i=1, \ldots, m .
$$

Contudo, não é simples obter um elemento de $\partial F\left(\lambda^{k+1}\right)$, para verificar a relação acima. De fato, a forma típica de se obter um subgradiente da função objetivo dual é através de uma minimização irrestrita do lagrangiano com o multiplicador fixo. Formalmente, sabemos que se

$$
\widehat{x} \in \underset{x \in \mathbb{R}^{n}}{\operatorname{Argmin}}\left\{f(x)+\left\langle\lambda^{k+1}, g(x)\right\rangle\right\},
$$

então

$$
g(\widehat{x}) \in \partial F\left(\lambda^{k+1}\right) .
$$

Usando este resultado, concluímos que o critério (6.5) será atendido se

$$
\left|g_{i}(\widehat{x})-g_{i}\left(x^{k+1}\right)\right| \leq \sigma\left|g_{i}\left(x^{k+1}\right)\right|, i=1, \ldots, m .
$$

Surge assim a necessidade de estimar o lado esquerdo desta desigualdade. 
Outros trabalhos, em particular [39, 5], também chegaram a condições de parada de difícil verificação. ${ }^{2}$ Para contornar os obstáculos encontrados, estes autores lançaram mão da hipótese de convexidade forte da função objetivo. Seguindo esta tradição, apesar de considerarmos esta condição restritiva, vamos assumir durante o restante desta seção que:

Hipótese 6.1.1. A função objetivo do problema primal, $f$, é fortemente convexa com módulo $\zeta$. Além disto as restrições, $g_{i}, i=1, \ldots, m$ são globalmente Lipschitz com constantes $L_{i}, i=1, \ldots, m$.

Destacamos que se conhecêssemos uma caixa limitada que contém as soluções primais a condição de Lipschitz continuidade não seria necessária. Neste caso, as restrições que formam a caixa poderiam ser tratadas pelo algoritmo que resolve o sub-problema penalizado, evitando o uso de penalidades para este tipo simples de restrição. A condição de Lipschitz continuidade estaria então automaticamente verificada, já que toda função convexa real é Lipschitz em compactos.

Vamos usar esta nova hipótese, podemos simplificar (6.7). com este objetivo, definimos

$$
\phi_{k}(x) \stackrel{\text { def }}{=} f(x)+\sum_{i=1}^{m} \alpha_{i}^{k} P_{i}\left(\frac{g_{i}(x)}{\alpha_{i}^{k}}, \lambda_{i}^{k}\right)
$$

A definição $\lambda^{k+1}$ garante que

$$
\nabla \phi_{k}\left(x^{k+1}\right)=\nabla_{x} \mathrm{~L}\left(x^{k+1}, \lambda^{k+1}\right) .
$$

Já a convexidade forte de $f$ assegura que $\mathrm{L}\left(\cdot, \lambda^{k+1}\right)$ também é fortemente convexa e com o mesmo módulo (乙). Portando, usando [41, Exercício 12.59],

$$
\begin{aligned}
\zeta\left|\widehat{x}-x^{k+1}\right|^{2} & \leq\left\langle\widehat{x}-x^{k+1}, \nabla_{x} \mathrm{~L}\left(\widehat{x}, \lambda^{k+1}\right)-\nabla_{x} \mathrm{~L}\left(x^{k+1}, \lambda^{k+1}\right)\right\rangle \\
& \leq\left|\widehat{x}-x^{k+1}\right|\left|\nabla_{x} \mathrm{~L}\left(\widehat{x}, \lambda^{k+1}\right)-\nabla_{x} L\left(x^{k+1}, \lambda^{k+1}\right)\right|,
\end{aligned}
$$

uma vez que $\widehat{x}$ minimiza $L\left(\cdot, \lambda^{k+1}\right), \nabla_{x} L\left(\widehat{x}, \lambda^{k+1}\right)=0$. Desta forma, as desigualdades acima implicam que

$$
\zeta\left|\widehat{x}-x^{k+1}\right| \leq\left|\nabla_{x} L\left(x^{k+1}, \lambda^{k+1}\right)\right|
$$

A Equação (6.9) nos diz que isto é equivalente a

$$
\zeta\left|\widehat{x}-x^{k+1}\right| \leq\left|\nabla \phi_{k}\left(x^{k+1}\right)\right|
$$

Finalmente, usamos a continuidade de Lipschitz das restrições para deduzir que para cada $i=1, \ldots, m$,

$$
\frac{\zeta}{\mathrm{L}_{i}}\left|g_{i}(\widehat{x})-g_{i}\left(x^{k+1}\right)\right| \leq\left|\nabla \phi_{k}\left(x^{k+1}\right)\right|
$$

\footnotetext{
${ }^{2}$ As condições destes artigos estavam ligadas a garantir $€$-otimalidade do ponto primal atual.
} 
E desta forma, o critério de aceitação (6.7) será satisfeito se

$$
\left|\nabla \phi_{k}\left(x^{k+1}\right)\right| \leq \frac{\zeta \sigma}{L_{i}}\left|g_{i}\left(x^{k+1}\right)\right|, i=1, \ldots, m
$$

Destacamos que este critério depende apenas dos dados primais e da solução aproximada do problema de minimização, sendo, desta forma, fácil de ser testado.

Portanto, a otimalidade dual dos pontos de acumulação de uma seqüência de multiplicadores obtida através de (6.2)-(6.4), usando (6.10) como critério de aceitação de uma solução aproximada do problema de minimização do lagrangiano aumentado, (6.3), é uma conseqüência do teorema de convergência do Algoritmo 4.3.1, Teorema 4.3.4. A limitação da seqüência de multiplicadores, mais uma vez, estará assegurada se a condição de qualificação de Slater for válida (Teorema 1.2.5).

\section{Testes Numéricos}

Fizemos alguns testes numéricos preliminares com o objetivo de verificar a adequação do critério de aceitação proposto em (6.10). Em particular, queremos verificar se a adoção desta condição de parada interfere na "capacidade de resolução de problemas" do algoritmo e se há algum ganho de desempenho quando comparado a uma implementação que busque resolver todos os sub-problemas com precisão elevada.

Implementamos então o método de multiplicadores baseado na penalidade logarítmica-quadrática, descrita na Seção 5.3. Para resolver os sub-problemas irrestritos usamos o L-BFGS-B, código de domínio público descrito em [15]. ${ }^{3}$ Este programa mostrou-se especialmente interessante por fazer comunicação reversa a cada passo, o que permitiu a incorporação de nosso novo critério de parada sem a necessidade de alteração do código original.

Os problemas de teste foram escolhidos na coleção de problemas de pequeno porte mastsif_small, que pode ser obtida junto com o CUTE [10]. Nela encontramos 130 problemas cujas restrições são todas de desigualdade ou limites nas variáveis. Uma lista completa destes problemas pode ser encontrada no Apêndice.

Buscamos resolver os problemas escolhidos com nossa implementação do Algoritmo 4.3.5. Um par $(x, \lambda)$ foi aceito como um par de soluções primal-dual se ocorrer:

1. Viabilidade.

$$
\max _{i=1, \ldots, m}\left\{\max \left(0, g_{i}(x)\right)\right\} \leq 10^{-6} .
$$

\footnotetext{
${ }^{3} \mathrm{O}$ código fonte deste programa está disponível na página http://www-c.mcs .anl.gov/home/otc/.
} 
2. Complementaridade.

$$
|\langle\lambda, g(x)\rangle| \leq 10^{-6} \max (f(x), 1.0)
$$

3. Mínimo do lagrangiano.

$$
\frac{\max _{i=1, \ldots, m}\left\{\left|\nabla_{x} L(x, \lambda)_{i}\right| \max \left(\left|x_{i}\right|, 1.0\right)\right\}}{\max (L(x, \lambda), 1.0)} \leq 10^{-6} .
$$

Cada problema foi abordado de duas formas. Primeiro, buscamos resolver todos os sub-problemas até que a norma do respectivo gradiente fosse próxima de zero. ${ }^{4}$ Chamaremos esta versão de exata. A segunda maneira usou o critério de aceitação (6.10) caso este se verifique antes que o gradiente torne-se muito pequeno. Chamaremos esta versão de inexata.

A versão exata resolveu 95 dos 130 problemas, já a inexata 92. Concluímos que, nesta amostra, a "capacidade de solução dos problemas" de ambos é, qualitativamente, a mesma. Dos 130 problemas, 90 foram resolvidos pelas duas versões implementadas. Destes, 9 foram resolvidos usando um único sub-problema, ou seja eram problemas irrestritos, ou o ponto dual inicial fornecido pelo CUTE era uma solução do dual. Usamos os 81 problemas restantes para um teste de desempenho.

A versão inexata resolveu estes 81 problemas em média em $77,6 \%$ do tempo da versão exata, computando o gradiente da função objetivo apenas $88,0 \%$ do número de vezes que este mesmo gradiente foi computado pela versão exata. Já os gradiente das restrições foram computados $88,9 \%$ vezes o número de cômputos destes gradientes feito pelo método exato. Assim, podemos dizer que a condição de parada apresentou-se como uma alternativa interessante para ganho de desempenho em métodos de multiplicadores.

Por outro lado, em apenas $59,3 \%$ dos casos o critério (6.10) foi capaz de garantir a aceitação de no mínimo $75 \%$ das soluções aproximadas dos problemas penalizados. Logo, é freqüente que o critério de aceitação degenere "rapidamente" na solução exata dos sub-problemas. Porém, mesmo nesta situação, a menor precisão exigida nas primeiras minimizações garante uma vantagem de desempenho.

Tabelas com os nomes de todos os problemas resolvidos e os parâmetros de desempenho para cada um deles estão presentes no Apêndice C.

\subsection{O Método Híbrido de Solodov e Svaiter}

Em [39], Rockafellar apresentou uma alternativa ao método de multiplicadores que chamou de método do lagrangiano aumentado proximal. Para obter este algoritmo, ele

\footnotetext{
${ }^{4}$ Menor ou igual a $10^{-8}$.
} 
aplicou o método de ponto proximal clássico à solução do problema de encontrar um ponto de sela do lagrangiano. O algoritmo obtido é um método primal-dual bastante semelhante ao método do lagrangiano aumentado clássico, porém com melhores propriedades de convergência.

Nesta seção, nós vamos aplicar o método híbrido de Solodov e Svaiter, apresentado na Seção 2.1, para obter uma versão do método do lagrangiano aumentado proximal com um critério de aceitação de soluções aproximadas de seus sub-problemas de fácil verificação e sem a necessidade de hipóteses restritivas sobre o problema que desejamos resolver. Esta seção é baseada no artigo [26].

Consideremos o problema primal, definido no Capítulo 1. A respectiva função lagrangiana estendida é dada por:

$$
L(x, \lambda) \stackrel{\text { def }}{=} f(x)+\langle\lambda, g(x)\rangle-\delta_{\mathbb{R}_{+}^{m}}(\lambda) .
$$

Como L é uma função convexa em $x$ e côncava em $\lambda$, podemos associar a ela um operador monótono maximal cujos zeros coincidem com seus pontos de sela [41, Exemplo 12.27]. Chamaremos este operador de $T_{L}$ e ele é definido por:

$$
\mathrm{T}_{\mathrm{L}}(x, \lambda) \stackrel{\text { def }}{=}\left[\begin{array}{c}
\nabla f(x)+\sum_{i=1}^{m} \lambda_{i} \nabla g_{i}(x) \\
-g(x)+N_{\mathbb{R}_{+}^{m}}(\lambda)
\end{array}\right] .
$$

$\mathbf{N}_{\mathbb{R}_{+}^{m}}(\lambda)$ é o cone normal associado a $\mathbb{R}_{+}^{m}$ em $\lambda$, cuja fórmula é:

$$
\mathbf{N}_{\mathbb{R}_{+}^{m}}(\lambda)_{i} \stackrel{\text { def }}{=} \begin{cases}\{0\} & \text { se } \lambda_{i}>0 ; \\ (-\infty, 0] & \text { se } \lambda_{i}=0 ; \\ \emptyset & \text { se } \lambda_{i}<0 .\end{cases}
$$

Por outro lado, os pontos de sela de L coincidem com os pares de soluções dos problemas primal e dual, uma vez que assumimos a validade de uma condição de qualificação [29, Teorema 5.4.7]. Podemos então aplicar um método proximal para encontrar zeros de $\mathrm{T}_{\mathrm{L}}$, e assim obter soluções do problema primal (e dual). 


\section{O Método Híbrido Projeção-Lagrangiano Aumentado Proximal}

Seguindo os passos de Rockafellar em [39], sabemos que o passo proximal do método híbrido aplicado a busca de zeros de $T_{L}$ pode ser aproximado por:

$$
\begin{aligned}
P(u, \lambda) & \stackrel{\text { def }}{=} \frac{1}{2} \sum_{i=1}^{m}\left[\left(\lambda_{i}+u_{i}\right)_{+}^{2}-\left(\lambda_{i}\right)^{2}\right] ; \mathbb{T} \\
0 & <\alpha_{k} \leq \widetilde{\alpha} ; \\
\widetilde{x}^{k} & \approx \underset{x \in \mathbb{R}^{n}}{\operatorname{Argmin}}\left\{f(x)+\alpha_{k} P\left(\frac{g(x)}{\alpha_{k}}, \lambda^{k}\right)+\frac{\alpha_{k}}{2}\left\|x-x^{k}\right\|^{2}\right\} ; \\
\widetilde{\lambda}^{k} & \stackrel{\text { def }}{=} \nabla_{u} P\left(\frac{g\left(\widetilde{x}^{k}\right)}{\alpha_{k}}, \lambda_{i}^{k}\right) \ldots
\end{aligned}
$$

No restante deste capítulo, vamos denotar a função de $x$ que é minimizada em (6.13) por $\phi_{k}(x)$.

Para aceitarmos esta aproximação é preciso que, dado $\sigma \in[0,1), \exists \widetilde{\gamma}^{k} \in \mathrm{T}_{\mathrm{L}}\left(\widetilde{x}^{k}, \widetilde{\lambda}^{k}\right)$,

$$
\left\|\widetilde{\gamma}^{k}+\alpha_{k}\left(\left[\begin{array}{c}
\tilde{x}^{k} \\
\tilde{\lambda}^{k}
\end{array}\right]-\left[\begin{array}{c}
x^{k} \\
\lambda^{k}
\end{array}\right]\right)\right\| \leq \sigma \max \left\{\left\|\widetilde{\gamma}^{k}\right\|, \alpha_{k}\left\|\left[\begin{array}{c}
\tilde{x}^{k} \\
\tilde{\lambda}^{k}
\end{array}\right]-\left[\begin{array}{c}
x^{k} \\
\lambda^{k}
\end{array}\right]\right\|\right\} .
$$

Entre os elementos de $T_{L}$ que podemos usar para verificar este critério de aceitação, aquele que nos parece mais interessante é:

Lema 6.2.1. Sejam $\tilde{x} \in \mathbb{R}^{n}, \lambda \in \mathbb{R}_{+}^{m}$ e $\alpha>0$. Defina

$$
\tilde{\lambda} \stackrel{\text { def }}{=} \nabla_{\mathrm{u}} \mathrm{P}\left(\frac{\mathrm{g}\left(\widetilde{x}^{\mathrm{k}}\right)}{\alpha_{\mathrm{k}}}, \lambda\right) e \widetilde{\eta} \stackrel{\text { def }}{=}\left(\lambda+\frac{\mathrm{g}(\widetilde{x})}{\alpha}\right)_{-},
$$

então

$$
\widetilde{\eta} \in N_{\mathbb{R}_{+}^{m}}(\widetilde{\lambda})
$$

Logo,

$$
\widetilde{\gamma} \stackrel{\text { def }}{=}\left[\begin{array}{c}
\nabla f(\widetilde{x})+\sum_{i=1}^{m} \widetilde{\lambda}_{i} \nabla g_{i}(\widetilde{x}) \\
-g(\widetilde{x})+\alpha \tilde{\eta}
\end{array}\right] \in T_{L}(\widetilde{x}, \tilde{\lambda}) .
$$

Demonstração. O resultado é uma conseqüência imediata de $\nabla_{u} P(u, \lambda)=(\lambda+u)_{+}$.

A escolha de $\widetilde{\eta}$ entre os elementos de $N_{\mathbb{R}_{+}^{m}}(\widetilde{\lambda})$ é justificada pelo seguinte lema:

đLembramos que a regularização usada para a obtenção do método híbrido foi o quadrado da norma euclidiana. 
Lema 6.2.2. Sejam $\widetilde{x}^{k} \in \mathbb{R}^{n}, \lambda^{k} \in \mathbb{R}_{+}^{m} e \alpha_{k}>0$. Definindo $\widetilde{\lambda}^{k}, \widetilde{\eta}^{k} e \widetilde{\gamma}^{k}$, como no lema anterior, temos:

$$
\tilde{\gamma}^{k}+\alpha_{k}\left(\left[\begin{array}{c}
\tilde{x}^{k} \\
\tilde{\lambda}^{k}
\end{array}\right]-\left[\begin{array}{c}
x^{k} \\
\lambda^{k}
\end{array}\right]\right)=\left[\begin{array}{c}
\nabla \phi_{k}\left(\tilde{x}^{k}\right) \\
0
\end{array}\right] .
$$

Demonstração. Usando a regra da cadeia e a definição de $\tilde{\lambda}^{k}$, segue que $\nabla \phi_{k}\left(\widetilde{x}^{k}\right)=$ $\nabla f(\tilde{x})+\sum_{i=1}^{m} \tilde{\lambda}_{i} \nabla g_{i}(\widetilde{x})+\alpha_{k}\left(\widetilde{x}^{k}-x^{k}\right)$.

Além do mais,

$$
\begin{aligned}
-g\left(\widetilde{x}^{k}\right)+\alpha_{k} \widetilde{\eta}^{k}+ & \alpha_{k}\left(\tilde{\lambda}^{k}-\lambda^{k}\right)= \\
=- & g\left(\widetilde{x}^{k}\right)+\alpha_{k} \min \left\{\lambda^{k}+g\left(\widetilde{x}^{k}\right) / \alpha_{k}, 0\right\}+ \\
& \left.+\alpha_{k}\left(\max \left\{\lambda^{k}+g\left(\widetilde{x}^{k}\right) / \alpha_{k}, 0\right\}-\lambda^{k}\right\}\right) \\
= & \alpha_{k}\left(\min \left\{\lambda^{k},-g\left(\widetilde{x}^{k}\right) / \alpha_{k}\right\}+\max \left\{g\left(\widetilde{x}^{k}\right) / \alpha_{k},-\lambda^{k}\right\}\right) \\
= & 0 .
\end{aligned}
$$

Isto completa a prova.

De posse destes lemas, podemos simplificar o critério de aceitação (6.15):

$$
\left\|\nabla \phi_{k}(\tilde{x})\right\| \leq \sigma \max \left\{\left\|\widetilde{\gamma}^{k}\right\|, \alpha_{k}\left\|\left[\begin{array}{c}
\tilde{x}^{k} \\
\tilde{\lambda}^{k}
\end{array}\right]-\left[\begin{array}{c}
x^{k} \\
\lambda^{k}
\end{array}\right]\right\|\right\} .
$$

O novo par de pontos primal-dual, $\left(x^{k+1}, \lambda^{k+1}\right)$ é obtido pela projeção de $\left(x^{k}, \lambda^{k}\right)$ sobre o hiperplano que passa por $\left(\widetilde{\chi}^{k}, \widetilde{\lambda}^{k}\right)$ e com normal dada por $\widetilde{\gamma}^{k}$, definida como no Lema 6.2.1.

Finalmente, o Teorema 2.1.8 garante a convergência de $\left\{\left(x^{k}, \lambda^{k}\right)\right\}$ a um zero de $T_{L}$, ou seja, a um par de soluções primal-dual.

\section{Testes Numéricos}

Usamos o mesmo programa e conjunto de teste descritos na seção anterior para analisar o sucesso do critério de aceitação do método híbrido projeção-lagrangiano aumentado proximal.

Poucas modificações foram necessárias no código fonte dos programas para adaptálo a este novo algoritmo. Em particular, mantivemos o L-BFGS-B como rotina para solução dos problemas irrestritos.

Como na seção anterior, temos duas versões do método do lagrangiano aumentado proximal. Uma, que chamamos exata, resolve todos os sub-problemas com grande precisão, a segunda, ou inexata, implementa o critério de aceitação (6.17). 
Dos 130 problemas presentes em nossa massa de testes, as versões exata e inexata resolveram 91 (não necessariamente os mesmos). Concluímos que a "capacidade de solução dos problemas" de ambos é, qualitativamente, a mesma. Já, 84 problemas foram resolvidos pelas duas versões. Destes, 8 eram problemas irrestritos, ou o ponto dual inicial fornecido pelo CUTE era uma solução do dual. Usamos os 76 problemas restantes para um teste de desempenho.

Os resultados dos testes de desempenho são, até um certo ponto inesperados. A versão inexata foi mais rápida, levando $88,5 \%$ do tempo da versão exata, em média. Porém ela perdeu em todos os outros parâmetros de desempenho: o número de vezes que os gradientes da função objetivo e das restrições foram computados foi $5 \%$ maior do que o que foi feito pela versão exata. Portanto, concluímos que nesta amostra não há ganho de desempenho com a aceitação antecipada das soluções dos sub-problemas.

Por outro lado, em 58 problemas $(69,0 \%)$, o critério de aceitação foi atingindo em pelo menos $75 \%$ das minimizações irrestritas. Desta forma, o critério descrito em (6.17) mostrou-se mais robusto em nossa amostra do que o critério da seção anterior. Mas observando as tabelas, vemos que nos problemas nos quais esse critério foi mal sucedido o desempenho da versão inexata foi bem pior do que o desempenho da versão exata. Acreditamos que é necessário um estudo mais aprofundado destes resultados para uma melhor compreensão dos casos nos quais o uso da versão inexata pode trazer ganhos de desempenho.

Mais uma vez, tabelas com os nomes de todos os problemas resolvidos e os parâmetros de desempenho para cada um deles estão presentes no Apêndice C. 


\section{Conclusão}

Neste trabalho, apresentamos contribuições a teoria de métodos proximais. Em particular, introduzimos duas novas classes de regularizações: uma baseada em translações de funções convexas e a outra composta de termos coercivos adaptados a solução de problemas em caixa. Provamos a convergência dos respectivos algoritmos sob variadas condições. Tais métodos podem ser usados para resolução de problemas de otimização convexa e desigualdades variacionais. Em particular, estendemos os resultados de Auslender, Teboulle e Ben-Tiba apresentados em [4, 5], de forma a incluir regularizações baseadas em distâncias de Bregman.

Desta forma, recuperamos os resultados sobre métodos inexatos de Solodov e Svaiter em [43], sem a necessidade de um passo extra-gradiente no caso de otimização convexa. Recentemente, encontramos um artigo de Birge, Qi e Wei [9] que contém o resultado de convergência sem passo extra-gradiente. Este artigo lida apenas com o caso de otimização e possui uma análise semelhante àquela apresentada no início da Seção 2.1, centrando o estudo na queda da função objetivo.

Por fim, apresentamos novas regras de aceitação de soluções aproximadas dos problemas irrestritos que surgem em alguns métodos de multiplicadores e fizemos alguns testes computacionais para validar estas regras.

Surgem destes resultados novas questões que merecem investigação, dentre as quais destacamos três, que são objeto presente de nossa pesquisa:

1. Estudo das penalidades geradas a partir de regularizações duplas, se possível explorando a conexão, estabelecida no final do Capítulo 5, com o artigo de Chen e Mangasarian [17].

2. Extensão da análise do método proximal baseado em distâncias coercivas (Algoritmo 4.2.1) de maneira a incluir €-extensões [13] de operadores monótonos. 
3. Adaptação do algoritmo acima para problemas com restrições lineares gerais (poliedros), preservando, se possível, seu critério de aceitação. 


\section{Existência de zeros de desigualdades variacionais regularizadas}

Vamos estudar o problema de existência de zeros de desigualdades variacionais regularizadas por um termo estritamente convexo. Deste modo, apresentaremos resultados que podem ser usados para provara existência de iterados proximais usando diferentes hipóteses sobre as distâncias generalizadas e/ou sobre operador T. Em particular estendemos o Lema 4.2.3 apresentado no Capítulo 4.

Dado uma desigualdade variacional (1.4), desejamos estudar a existência de soluções para

$$
0 \in \mathrm{T}\left(x^{*}\right)+\mathrm{N}_{\mathrm{B}}\left(x^{*}\right)+\partial \mathrm{D}\left(x^{*}\right)
$$

na qual

Hipótese A.1.1. A função D : $\mathbb{R}^{n} \rightarrow(-\infty, \infty]$ é fechada e estritamente convexa. Ainda, $\mathrm{D}$ é essencialmente lisa ${ }^{1}$, int dom $\mathrm{D}=$ int $\mathrm{B}$ e $\mathrm{D}$ possui mínimo em int $\mathrm{B}$.

Claramente, estas regularizações englobam as distâncias coercivas apresentadas no Capítulo 4, $\varphi$-divergências e distâncias de Bregman com zona int $B$.

Provamos inicialmente que:

Lema A.1.2. Qualquer solução de (A.1) está em intB.

Demonstração. Isto é uma conseqüência trivial do fato de D ser essencialmente lisa e int $\operatorname{dom} \mathrm{D}=\operatorname{int} \mathrm{B}$.

Passamos a apresentar condições que assegurem a existência de zeros (A.1).

\footnotetext{
${ }^{1} \mathrm{D}$ é diferenciável no interior de dom $D$, que é não vazio, e $\partial \mathrm{D}=\emptyset$ em todos os pontos $x \in \operatorname{dom} D$ int dom D.
} 
Lema A.1.3. Se rge $\mathrm{D}=\mathbb{R}^{\mathrm{n}}$, então existe um ponto $x^{*} \in \mathbb{R}^{n}$ que resolve (A.1). ${ }^{2}$

Demonstração. Como dom $T \cap \operatorname{int} B \neq \emptyset$, sabemos que deve existir um ponto $\bar{x} \in$ ri dom $T \cap \operatorname{int} B$. Portanto $\bar{x} \in$ ri $\operatorname{dom}\left(T+N_{B}\right)$. Além disto, como dom $\partial D=\operatorname{int} B$, concluímos que $T+N_{B}+\partial D$ é monótono maximal.

Portanto, usamos [35, Proposition 3] para concluir que

$$
\operatorname{rge}\left(T+N_{B}+\partial D\right)=\mathbb{R}^{n} \text {. }
$$

Lema A.1.4. Se T é o subdiferencial de uma função, $\mathrm{f}$, própria, fechada, convexa e limitada inferiormente em $\mathrm{B}$, então existe um ponto $x^{*} \in \mathbb{R}^{n}$ que resolve (A.1).

Demonstração. Neste caso (A.1) é equivalente a $f+D$ possuir ponto de mínimo em $B$. Para mostrar isto, basta provarmos que os conjuntos de nível desta função são limitados em B. Seja $l$ um limite inferior de $f$ em B. Dado qualquer $\alpha \in \mathbb{R}$ :

$$
\begin{aligned}
\{x \in \mathbb{R} \mid f(x)+D(x) \leq \alpha\} \cap B & =\{x \in B \mid f(x)+D(x) \leq \alpha\} \\
& \supset\{x \in B \mid D(x) \leq \alpha-l\} .
\end{aligned}
$$

O último conjunto é um conjunto de nível de $D$ em $B$, que é limitado pois $D$ possui um único mínimo em B.

Lema A.1.5. Seja

$$
h_{T, B}(x) \stackrel{\text { def }}{=} \sup \{\langle v, x-y\rangle \mid y \in B, v \in T(y)\} .
$$

Se $h_{T, \mathrm{~B}}(\mathrm{x})<\infty$ para todo $\mathrm{x} \in \mathrm{B} \cap \operatorname{dom} \mathrm{T}$, então existe um ponto $\mathrm{x}^{*} \in \mathbb{R}^{\mathrm{n}}$ que resolve (A.1).

Antes de apresentarmos a prova deste lema, destacamos que a hipótese $h_{\mathrm{T}, \mathrm{B}}(x)<\infty$ para todo $x \in B \cap \operatorname{dom} T$ já apareceu na literatura, por exemplo em [35, Teorema 2] e [14]. Em particular, a Proposição 3.1 de [14] garante que esta condição vale sempre que:

1. T é coerciva;

2. T é o subdiferencial de uma função própria, fechada, convexa e limitada inferiormente em B. Portanto, este Lema generaliza o Lema A.1.4;

\footnotetext{
${ }^{2}$ Este é, na verdade, o Lema 4.2.3, porém decidimos repetí-lo para que a exposição seja completa.
} 
3. $T+N_{B}$ é regular e possui zeros;

4. T é fortemente monótono;

5. $\overline{\operatorname{dom} T}$ é limitado;

Demonstração. (do Lema A.1.5). Seja $\bar{x}$ o ponto de mínimo de D em B. Como D é subdiferenciável em $\bar{x}, \bar{x} \in$ int $B$.

Seja $\alpha=\left|h_{T, B}(\bar{x})\right|$. Como os conjuntos de nível de $D$ são limitados, sabemos que deve existir um $\rho>0$ tal que, se $\|x-\bar{x}\| \geq \rho$, então

$$
\mathrm{D}(\mathrm{x})-\mathrm{D}(\overline{\mathrm{x}}) \geq \alpha \text {. }
$$

Sejam $x, v \in \mathbb{R}^{n}$ tais que $\|x-\bar{x}\| \geq \rho$, e $v \in\left(T+N_{B}+\partial D\right)(x)$. Como $\partial D(x) \neq \emptyset$, sabemos que $x \in$ int $B$, portanto $N_{B}(x)=\{0\}$. Assim, $v$ pode ser escrito na forma $v_{\mathrm{T}}+v_{\mathrm{D}}$, para $v_{\mathrm{T}} \in \mathrm{T}(\mathrm{x})$ e $v_{\mathrm{D}} \in \partial \mathrm{D}(\mathrm{x})$. Logo,

$$
\begin{aligned}
\langle\nu, x-\bar{x}\rangle & =\left\langle\nu_{\mathrm{T}}, x-\bar{x}\right\rangle+\left\langle\nu_{\mathrm{D}}, x-\bar{x}\right\rangle \\
& \geq-\alpha+\left\langle\nu_{\mathrm{D}}, x-\bar{x}\right\rangle \\
& \geq-\alpha+\alpha \\
& =0 .
\end{aligned}
$$

Concluímos, usando o [41, Teorema 12.51] que $T+N_{B}+\partial D$ possui um zero.

O próximo resultado possui uma prova mais sofisticada, mas parece novo. Em particular, ele garante a existência de iterados para qualquer método proximal baseado em distâncias de Bregman que assuma que a função de Bregman é finita em B (o fecho da zona). Este é o caso em $[19,35,20]$, logo provamos que hipóteses do tipo "rge $h^{\prime}=\mathbb{R}^{n \text { " }}$ não seriam necessárias.

Iniciamos relembrando um resultado de Brézis e Haraux. Denotaremos por conv A o casco convexo de um conjunto $A$.

Lema A.1.6. [12, Lema 1] Seja $\mathrm{H}$ um espaço de Hilbert. Seja C um operado monótono maximal em $\mathrm{H}$ e $\mathrm{F} \subset \mathrm{H}$ tais que

$\forall v \in \mathrm{F}, \exists x_{v} \in \mathrm{H}$ tal que

$$
\sup \left\{\left\langle u-v, x_{v}-x\right\rangle \mid x \in \operatorname{dom} C, u \in C(x)\right\}<+\infty .
$$

Então, conv $\mathrm{F} \subset \overline{\operatorname{rge} C} e \operatorname{int}(\operatorname{conv} F) \subset \operatorname{rge} C$.

Podemos agora apresentar o nosso resultado. A demonstração apresentada é uma generalização, mais ou menos direta, da prova do Teorema A.1 de [2]. 
Lema A.1.7. Se $0 \in \overline{\operatorname{rge}\left(\mathrm{T}+\mathrm{N}_{\mathrm{B}}\right)}$ e para todo $\mathrm{y} \in \operatorname{int} \mathrm{B}, w \in \mathrm{B}$

$$
\sup _{z \in \operatorname{int} B}\{\langle\nabla D(z)-\nabla D(y), w-z\rangle\}<+\infty .
$$

Então, existe um ponto $\chi^{*} \in \mathbb{R}^{n}$ que resolve (A.1). Ainda, se D for finita em B, então (A.3) é válida.

Demonstração. A prova está dividida em três partes:

1. $\overline{\operatorname{rge}\left(T+N_{B}+\partial D\right)} \supset \overline{\operatorname{rge}\left(T+N_{B}\right)}+\overline{\operatorname{rge} \partial D}$.

Vamos aplicar o Lema A.1.6 com $F=\operatorname{rge}\left(T+N_{B}\right)+\operatorname{rge} \partial D$ e $C=T+N_{B}+\partial D$. Uma vez que $\operatorname{dom}\left(T+N_{B}+\partial D\right)=\operatorname{dom} T \cap \operatorname{int} B$, devemos provar que

$$
\begin{aligned}
& \forall v \in \operatorname{rge}\left(T+N_{B}\right)+\operatorname{rge} \partial D, \exists x_{v} \in \mathbb{R}^{n} \text { tal que } \\
& \quad \sup \left\{\left\langle u-v, x_{v}-x\right\rangle \mid x \in \operatorname{dom} T \cap \operatorname{int} B, u \in(T+\nabla D)(x)\right\}<+\infty
\end{aligned}
$$

Dado $v \in \operatorname{rge}\left(T+N_{B}\right)+\operatorname{rge} \partial D$, sejam $v_{1}, \nu_{2} \in \mathbb{R}^{n}, x_{v} \in B$ e $\tilde{x}_{v} \in \operatorname{int} B$ tais que:

$$
v=v_{1}+v_{2}, v_{1} \in\left(T+N_{B}\right)\left(x_{v}\right), v_{2} \in \nabla D\left(\widetilde{x}_{v}\right) .
$$

E sejam $x \in \operatorname{dom} T \cap$ int $B$ e $u \in(T+\nabla D)(x)$, tais que $u=u_{1}+u_{2}$ para algum $u_{1} \in T(x)$ e $u_{2} \in \nabla D(x)$.

Temos

$$
\begin{array}{rlr}
\left\langle u-v, x_{v}-x\right\rangle & =\left\langle u_{1}+u_{2}-v_{1}-v_{2}, x_{v}-x\right\rangle & \\
& =\left\langle u_{1}-v_{1}, x_{v}-x\right\rangle+\left\langle u_{2}-v_{2}, x_{v}-x\right\rangle & \\
& \leq\left\langle u_{2}-v_{2}, x_{v}-x\right\rangle & \\
& =\left\langle\nabla D(x)-\nabla D\left(\widetilde{x}_{v}\right), x_{v}-x\right\rangle &
\end{array}
$$

Tomando o supremo em $x \in \operatorname{dom} T$ nint B, podemos usar (A.3) e concluir que (A.4) vale.

Deste modo, aplicando o Lema A.1.6, segue que

$$
\begin{aligned}
& \overline{\operatorname{rge}\left(T+N_{B}+\partial D\right)} \supset \operatorname{conv}\left(\operatorname{rge}\left(T+N_{B}\right)+\operatorname{rge} \partial D\right) \Longrightarrow \\
& \overline{\operatorname{rge}\left(T+N_{B}+\partial D\right)} \supset \overline{\operatorname{rge}\left(T+N_{B}\right)+\operatorname{rge} \partial D} \\
& \supset \overline{\operatorname{rge}\left(T+N_{B}\right)}+\overline{\text { rge } \partial D} \text {. }
\end{aligned}
$$


2. Seja $\delta_{K}^{*}(\cdot)$ a função suporte de um conjunto K:

$$
\delta_{\mathrm{K}}^{*}(d) \stackrel{\text { def }}{=} \sup _{x \in \mathrm{K}}\{\langle x, d\rangle\}
$$

Desejamos estimar

$$
\begin{array}{rlr}
\delta_{\mathrm{rge}\left(T+N_{B}+\partial D\right)}^{*}(d) & \geq \delta_{\frac{\operatorname{rge}\left(T+N_{B}\right)}{*}}^{*} \frac{}{\operatorname{rge} \partial D}(d) & \text { [ítem anterior] } \\
& =\delta_{\frac{\operatorname{rge}\left(T+N_{B}\right)}{*}}^{*}(d)+\delta_{\text {rge } \partial D}^{*}(d) \\
& \geq \delta_{\text {rge } \partial D}^{*}(d),
\end{array}
$$

uma vez que $0 \in \overline{\operatorname{rge}\left(T+N_{B}\right)}$.

Por outro lado, seja $\bar{x}$ o ponto de int $B$ que minimiza D. Dado $d \neq 0$, seja $\alpha$ um número estritamente positivo pequeno o suficiente para que $\bar{x}+\alpha d \in$ int $B$. Temos:

$$
\begin{aligned}
\alpha \delta_{\text {rge } \partial \mathrm{D}}^{*}(\mathrm{~d}) & \geq\left\langle\nabla \mathrm{D}\left(\mathrm{x}^{*}+\alpha \mathrm{d}\right), \alpha \mathrm{d}\right\rangle \\
& \geq \mathrm{D}\left(x^{*}+\alpha \mathrm{d}\right)-\mathrm{D}\left(x^{*}\right) \\
& >0
\end{aligned}
$$

Desta forma,

$$
\forall \mathrm{d} \neq 0, \delta_{\mathrm{rge}\left(\mathrm{T}+\mathrm{N}_{\mathrm{B}}+\partial \mathrm{D}\right)}^{*}(\mathrm{~d})>0 .
$$

Como $\overline{\operatorname{rge}\left(T+N_{B}+\partial D\right)}$ é um conjunto convexo [41, Teorema 12.41], podemos usar [37, Teorema 13.1] e concluir que

$$
0 \in \operatorname{int} \overline{\operatorname{rge}\left(T+N_{B}+\partial D\right)} \text {. }
$$

Mais uma vez por [41, Teorema 12.41], observamos que int $\overline{\operatorname{rge}\left(T+N_{B}+\partial D\right)}$ também é convexo e coincide com int rge $\left(T+N_{B}+\partial D\right)$. Portanto,

$$
0 \in \operatorname{intrge}\left(T+N_{B}+\partial D\right) \text {. }
$$

3. Finalmente provamos que se A.3 vale sempre que $D$ for finita em B. Seja $y \in$ int $\mathrm{B}, w \in \mathrm{B}$. Para todo $z \in \operatorname{int} \mathrm{B}$ :

$$
\begin{aligned}
\langle\nabla \mathrm{D}(z)- & \nabla \mathrm{D}(\mathrm{y}), w-z\rangle \leq \mathrm{D}(w)-\mathrm{D}(z)-\langle\nabla \mathrm{D}(\mathrm{y}), w-z\rangle \\
& =\mathrm{D}(w)-\mathrm{D}(z)-\langle\nabla \mathrm{D}(\mathrm{y}), w\rangle+\langle\nabla \mathrm{D}(\mathrm{y}), z\rangle \\
& \leq \mathrm{D}(w)-\mathrm{D}(z)-\langle\nabla \mathrm{D}(\mathrm{y}), w\rangle+\mathrm{D}(z)-\mathrm{D}(\mathrm{y})+\langle\nabla \mathrm{D}(\mathrm{y}), y\rangle \\
& =\mathrm{D}(w)-\mathrm{D}(\mathrm{y})+\langle\nabla \mathrm{D}(\mathrm{y}), y-w\rangle .
\end{aligned}
$$

Como o lado-direito não depende de $z$, o supremos é finito.

A prova está completa.

Outras regularizações importantes que obedecem a A.3 são as "distâncias de Bregman" e $\varphi$-divergências obtidas a partir de $-\log x$ [2, Apêndice A]. 


\section{APÊNDICE B}

\section{Conexões entre distâncias de Bregman e $\varphi$-divergências}

Neste apêndice, vamos discutir algumas conexões entre distâncias de Bregman e $\varphi$ divergências com mudança de escala, descrita na Seção 4.1.

Em [28], os autores destacam que a única regularização que é, ao mesmo tempo, uma distância de Bregman e uma $\varphi$-divergência é a distância de Kullback-Leibler, dada por

$$
d(x, y)=x \log \frac{x}{y}+y-x .
$$

Esta regularização é a distância de Bregman associada a $h(x)=x \ln x$ e a $\varphi$-divergência associada a $\varphi(t)=t \log t-t+1$.

Por outro lado, ao empregarmos a técnica de mudança de escala a diversas outras regularizações, observamos que o número de $\varphi$-divergências correspondendo a distâncias de Bregman é bem maior. Até o momento observamos que, após a divisão pela segunda derivada, as seguintes regularizações coincidem:

1. Distância de Bregman obtida de $h(x)=x \log x$ e $\varphi$-divergência baseada em $\varphi(x)=x \log x-x+1$

2. Distância de Bregman obtida de $h(x)=-\log x$ e $\varphi$-divergência baseada em $\varphi(t)=-\log x+x-1$

3. Distância de Bregman obtida de $h(x)=1 / x$ e $\varphi$-divergência baseada em $\varphi(t)=$ $1 / 2(1 / x+x-2)$

4. Distância de Bregman obtida de $h(x)=x-\sqrt{x}$ e $\varphi$-divergência baseada em $\varphi(t)=2(\sqrt{x}-1)^{2}$ 


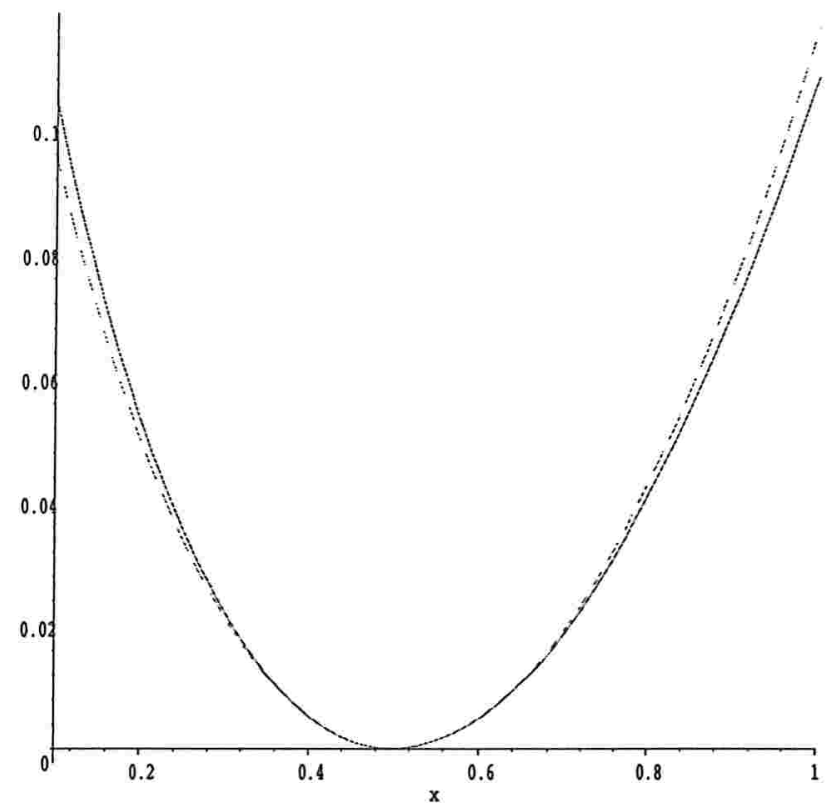

Figura B.1: Mudança de escala atuando na distância de Bregman (linha contínua) e na $\varphi$-divergência (linha tracejada) baseadas em $h(x)=x \log \left(e^{x}-1\right)$, com mudança de escala e centro em 0.5.

Por outro lado, é claro, existem casos onde não é possível estabelecer esta correlação. Dada uma função de Bregman, $h$, para que os dois tipos de regularizações coincidam é preciso que:

$$
\frac{h(x)-h(1)-h^{\prime}(1)(x-1)}{h^{\prime \prime}(1)}=\frac{\varphi(x)}{\varphi^{\prime \prime}(1)} \text {. }
$$

Assim, temos uma maneira de determinar o único núcleo $\varphi$ que pode gerar uma $\varphi$ divergência que coincida com a distância de Bregman gerada a partir de $h$.

Consideremos $h(x)=x \log \left(e^{x}-1\right)$ e seja $\varphi$ obtida de $h$ da forma descrita acima. Podemos então usar a técnica de mudança de escala e estudar as regularizações coercivas baseadas nas respectivas distâncias de Bregman e $\varphi$-divergência. A Figura B.1 mostra seus gráficos com o centro fixado em 0,5 . Notamos que a distância que domina muda de acordo com o lado com relação ao centro e, portanto, as regularizações não coincidem. Outros exemplo de funções $h$ gerando distâncias generalizadas diferentes são $h(x)=$ $\operatorname{dilog}\left(e^{x}\right)+x \log \left(e^{x}-1\right)\left(\right.$ Figura B.2) e $h(x)=x^{2}-\sqrt{x}$ (Figura B.3). 


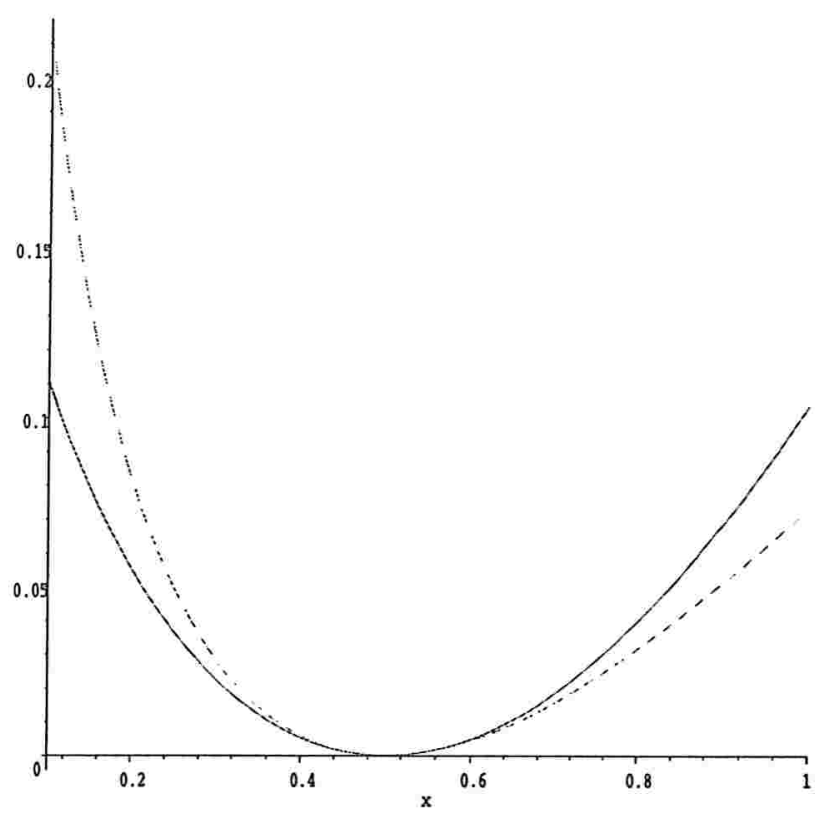

Figura B.2: Mudança de escala atuando na distância de Bregman (linha contínua) e na $\varphi$-divergência (linha tracejada) baseadas em $h(x)=\operatorname{dilog}\left(e^{x}\right)+x \log \left(e^{x}-1\right)$, com mudança de escala e centro em 0.5 .

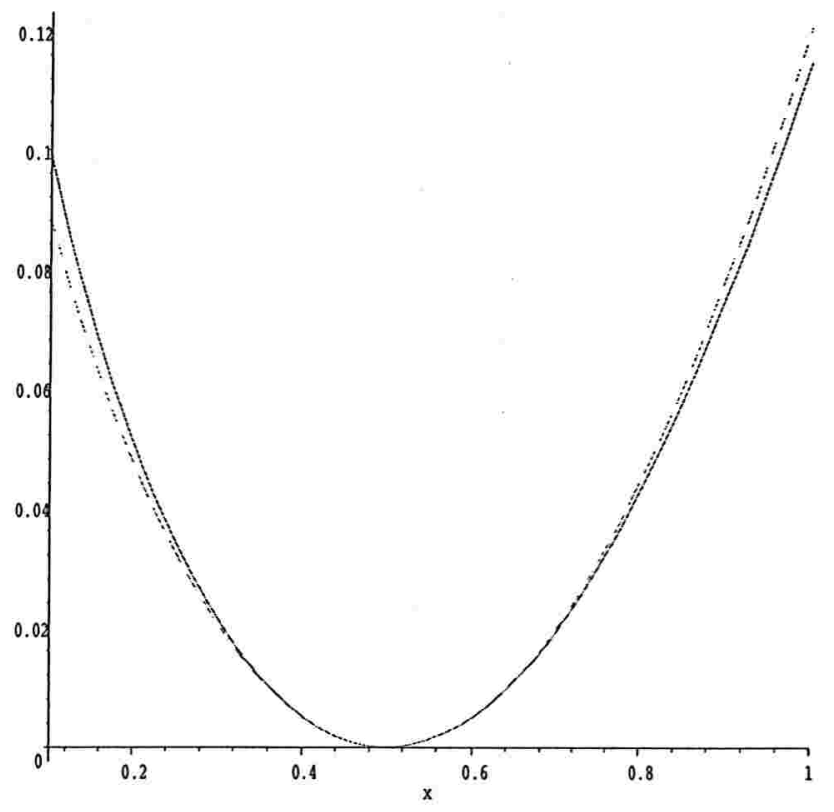

Figura B.3: Mudança de escala atuando na distância de Bregman (linha contínua) e na $\varphi$-divergência (linha tracejada) baseadas em $h(x)=x^{2}-\sqrt{x}$, com mudança de escala e centro em 0.5 . 
Terminamos esta seção com um Teorema, gentilmente cedido pelo Prof. Iusem em uma comunicação pessoal, que caracteriza totalmente a intersecção das duas classes de regularizações.

Consideremos $[\mathrm{a}, \mathrm{b})=\mathbb{R}_{+}$e $\operatorname{sejam} \varphi$ e $h$ funções obedecendo às Hipóteses 4.1.2 e 4.1.4 respectivamente. Supomos ainda que $\varphi$ é continuamente duas vezes diferenciável no $\mathbb{R}_{++}$. Denotamos por $d_{\varphi}$ a $\varphi$-divergência com mudança de escala obtida a partir de $\varphi$, dada por (4.4), e $d_{h}$ a distância de Bregman com mudança de escala obtida a partir de $h$, dada por (4.2).

Teorema B.1.1. Se para todo $x, y \in \mathbb{R}_{++}$

$$
d_{\varphi}(x, y)=d_{h}(x, y)
$$

então existe $\nu \in \mathbb{R}$ tal que

$$
\varphi^{\prime \prime}(x)=\varphi^{\prime \prime}(1) x^{\nu} .
$$

Ainda, se definirmos $\delta \stackrel{\text { def }}{=} h^{\prime \prime}(1) / \varphi^{\prime \prime}(1)$, existem $\alpha$ e $\beta$ tais que

$$
h(x)=\delta \varphi(x)+\alpha x+\beta .
$$

Demonstração. Derivando (B.1) dos dois lados temos:

$$
\frac{y}{\varphi^{\prime \prime}(1)} \varphi^{\prime}\left(\frac{x}{y}\right)=\frac{h^{\prime}(x)-h^{\prime}(y)}{h^{\prime \prime}(y)} .
$$

Tomando $y=1$ e $\alpha=h^{\prime}(1)$, temos

$$
h^{\prime}(x)=\delta \varphi^{\prime}(x)+\alpha .
$$

Assim, (B.3) segue por integração.

Substituindo então (B.3) em (B.1) e derivando duas vezs com relação a $x$ temos:

$$
\frac{\varphi^{\prime \prime}(x / y)}{\varphi^{\prime \prime}(1)}=\frac{\varphi^{\prime \prime}(x)}{\varphi^{\prime \prime}(y)}
$$

Fazendo $s=x / y$, chegamos a

$$
\varphi^{\prime \prime}(s) \varphi^{\prime \prime}(y)=\varphi^{\prime \prime}(s y) \varphi^{\prime \prime}(1) .
$$

Definimos então

$$
\begin{array}{r}
\psi(y) \stackrel{\text { def }}{=} \log \varphi^{\prime \prime}\left(e^{y}\right) \\
\rho(y) \stackrel{\text { def }}{=} \psi(y)-\psi(0) .
\end{array}
$$


Usando (B.4) e as definições obtemos facilmente que

$$
\rho(s+y)=\rho(s)+\rho(y) .
$$

Como $\varphi^{\prime \prime}$ é contínua, $\rho$ também o é e, portanto, (B.5) garante que $\rho$ é linear. Assim, $\psi$ é afim, ou seja, existem $\mu, v \in \mathbb{R}$ tais que $\psi(y)=\mu+v y$. Invertendo a definição de $\psi$ temos que $\varphi^{\prime \prime}(y)=e^{\mu} y^{\nu}, \operatorname{com} \varphi^{\prime \prime}(1)=e^{\mu}$, ou seja (B.2).

Temos em nossas mãos ferramentas suficientes para apresentar as possíveis expressões explícitas para $\varphi$ e h:

Corolário B.1.2. Se para todo $x, y \in \mathbb{R}_{++}$

$$
d_{\varphi}(x, y)=d_{h}(x, y),
$$

então vale um dos seguintes casos:

1. Existe $\gamma \in \mathbb{R}_{++}$tal que

$$
\varphi(x)=\gamma(x \log x-x+1)
$$

e existem $\lambda, \eta, \theta \in \mathbb{R}$ tais que

$$
h(x)=\lambda x \log x+\eta x+\theta .
$$

2. Existe $\gamma \in \mathbb{R}_{++}$tal que

$$
\varphi(x)=\gamma(-\log x+x-1)
$$

e existem $\lambda, \eta, \theta \in \mathbb{R}$ tais que

$$
h(x)=-\lambda \log x+\eta x+\theta .
$$

3. Existem $\gamma \in \mathbb{R}_{++} e \sigma \in \mathbb{R}, \sigma \neq 0,1$, tais que

$$
\varphi(x)=\frac{\gamma}{\sigma(\sigma-1)}\left(t^{\sigma}-\sigma t+\sigma-1\right)
$$

e existem $\lambda, \eta, \theta \in \mathbb{R}$ tais que

$$
h(x)=\lambda x^{\sigma}+\eta x+\theta .
$$

Demonstração. As fórmulas para $\varphi$ vêm da integração de (B.2) para $v=-1, v=-2$ e $v \neq-1,-2$ respectivamente. As fórmulas de $h$ seguem então de (B.3). 


\section{Tabelas dos Testes Numéricos}

Seguem as tabelas dos testes numéricos descritos no Capítulo 6. Iniciamos pela tabela com todos os problemas de teste.

$\begin{array}{llllll}\text { AIRPORT } & \text { ALLINITC } & \text { AVGASA } & \text { AVGASB } & \text { CANTILVR } & \text { CB2 } \\ \text { CB3 } & \text { CHACONN1 } & \text { CHACONN2 } & \text { CONGIGMZ } & \text { CRESC100 } & \text { CRESC4 } \\ \text { CRESC50 } & \text { DEMYMALO } & \text { DIPIGRI } & \text { ELATTAR } & \text { EQC } & \text { EXPFITA } \\ \text { EXPFITB } & \text { GIGOMEZ1 } & \text { GIGOMEZ2 } & \text { GIGOMEZ3 } & \text { GOFFIN } & \text { HAIFAM } \\ \text { HAIFAS } & \text { HALDMADS } & \text { HIMMELBI } & \text { HIMMELP2 } & \text { HIMMELP3 } & \text { HIMMELP4 } \\ \text { HIMMELP5 } & \text { HIMMELP6 } & \text { HS10 } & \text { HS100 } & \text { HS100MOD } & \text { HS105 } \\ \text { HS106 } & \text { HS108 } & \text { HS11 } & \text { HS113 } & \text { HS117 } & \text { HS12 } \\ \text { HS13 } & \text { HS15 } & \text { HS16 } & \text { HS17 } & \text { HS18 } & \text { HS19 } \\ \text { HS20 } & \text { HS21 } & \text { HS21MOD } & \text { HS22 } & \text { HS23 } & \text { HS24 } \\ \text { HS268 } & \text { HS29 } & \text { HS30 } & \text { HS31 } & \text { HS33 } & \text { HS34 } \\ \text { HS35 } & \text { HS35I } & \text { HS35MOD } & \text { HS36 } & \text { HS37 } & \text { HS43 } \\ \text { HS44 } & \text { HS44NEW } & \text { HS57 } & \text { HS59 } & \text { HS64 } & \text { HS65 } \\ \text { HS66 } & \text { HS70 } & \text { HS72 } & \text { HS76 } & \text { HS76I } & \text { HS86 } \\ \text { HS88 } & \text { HS89 } & \text { HS90 } & \text { HS91 } & \text { HS92 } & \text { HS93 } \\ \text { HS95 } & \text { HS96 } & \text { HS97 } & \text { HS98 } & \text { HUBFIT } & \text { KIWCRESC } \\ \text { LHAIFAM } & \text { LOOTSMA } & \text { LSQFIT } & \text { MADSEN } & \text { MAKELA1 } & \text { MAKELA2 } \\ \text { MAKELA3 } & \text { MAKELA4 } & \text { MATRIX2 } & \text { MIFFLIN1 } & \text { MIFFLIN2 } & \text { MINMAXBD } \\ \text { MINMAXRB } & \text { MISTAKE } & \text { OPTPRLOC } & \text { POLAK1 } & \text { POLAK2 } & \text { POLAK3 } \\ \text { POLAK4 } & \text { POLAK5 } & \text { POLAK6 } & \text { PRIMAL1 } & \text { PRIMALC1 } & \text { PRIMALC2 } \\ \text { PRIMALC5 } & \text { QC } & \text { QCNEW } & \text { ROSENMMX } & \text { S268 } & \text { S365 } \\ \text { SIMPLLPA } & \text { SIMPLLPB } & \text { SNAKE } & \text { SYNTHES1 } & \text { TWOBARS } & \text { WOMFLET } \\ \text { ZECEVIC2 } & \text { ZECEVIC3 } & \text { ZECEVIC4 } & \text { ZY2 } & & \end{array}$

Tabela C.1: Conjunto de 130 problemas com restrições de desigualdades da coleção do CUTE/mastsif_small [10] que serviu de base para os testes. 


\section{C.1 Método Multiplicadores com Distâncias Coer- civas (Algoritmo 4.3.5)}

Entre testes da Tabela C.1, as versões exatas e inexatas do Algoritmo 4.3.5 resolveram os subconjuntos de problemas dados nas tabelas a seguir. Apresentamos também a tabela com os 81 problemas usados na comparação entre os métodos.

$\begin{array}{llllll}\text { AIRPORT } & \text { AVGASA } & \text { AVGASB } & \text { CB2 } & \text { CHACONN1 } & \text { CONGIGMZ } \\ \text { DEMYMALO } & \text { DIPIGRI } & \text { EXPFITA } & \text { EXPFITB } & \text { GIGOMEZ1 } & \text { GIGOMEZ2 } \\ \text { HAIFAS } & \text { HIMMELBI } & \text { HIMMELP } & \text { HIMMELP4 } & \text { HIMMELP5 } & \text { HIMMELP6 } \\ \text { HS10 } & \text { HS100 } & \text { HS100MOD } & \text { HS105 } & \text { HS108 } & \text { HS11 } \\ \text { HS113 } & \text { HS117 } & \text { HS12 } & \text { HS15 } & \text { HS16 } & \text { HS17 } \\ \text { HS18 } & \text { HS20 } & \text { HS21 } & \text { HS21MOD } & \text { HS22 } & \text { HS23 } \\ \text { HS24 } & \text { HS29 } & \text { HS30 } & \text { HS31 } & \text { HS33 } & \text { HS34 } \\ \text { HS35 } & \text { HS35I } & \text { HS35MOD } & \text { HS36 } & \text { HS37 } & \text { HS43 } \\ \text { HS44 } & \text { HS44NEW } & \text { HS57 } & \text { HS59 } & \text { HS65 } & \text { HS66 } \\ \text { HS70 } & \text { HS72 } & \text { HS76 } & \text { HS76I } & \text { HS86 } & \text { HS89 } \\ \text { HS91 } & \text { HS95 } & \text { HS96 } & \text { HS97 } & \text { HS98 } & \text { HUBFIT } \\ \text { KIWCRESC } & \text { LSQFIT } & \text { MADSEN } & \text { MAKELA1 } & \text { MAKELA2 } & \text { MAKELA3 } \\ \text { MAKELA4 } & \text { MATRIX2 } & \text { MIFFLIN1 } & \text { MIFFLIN2 } & \text { MINMAXBD } & \text { MINMAXRB } \\ \text { MISTAKE } & \text { POLAK4 } & \text { POLAK5 } & \text { POLAK6 } & \text { PRIMAL1 } & \text { PRIMALC5 } \\ \text { QC } & \text { ROSENMMX } & \text { SIMPLLPA } & \text { SIMPLLPB } & \text { SYNTHES1 } & \text { TWOBARS } \\ \text { WOMFLET } & \text { ZECEVIC2 } & \text { ZECEVIC3 } & \text { ZECEVIC4 } & \text { ZY2 } & \end{array}$

Tabela C.2: Problemas resolvidos pela versão exata do Algoritmo 4.3.5

\begin{tabular}{|c|c|c|c|c|c|}
\hline AIRPORT & AVGASA & AVGASB & CB2 & CHACONN1 & CONGIGMZ \\
\hline DEMYMALO & DIPIGRI & EXPFITA & EXPFITB & GIGOMEZ1 & GIGOMEZ2 \\
\hline HAIFAS & HIMMELBI & HIMMELP2 & HIMMELP3 & HIMMELP4 & HIMMELP5 \\
\hline HIMMELP6 & HS10 & HS100 & HS100MOD & HS105 & HS108 \\
\hline HS11 & HS117 & HS12 & HS15 & HS16 & HS17 \\
\hline HS18 & HS19 & HS20 & HS21 & HS21MOD & HS22 \\
\hline HS23 & HS24 & HS29 & HS30 & HS31 & HS33 \\
\hline HS34 & HS35 & HS35I & HS35MOD & HS36 & HS37 \\
\hline HS43 & HS44 & HS44NEW & HS65 & HS66 & HS72 \\
\hline HS76 & HS76I & HS86 & HS91 & HS95 & HS96 \\
\hline HS97 & HS98 & HUBFIT & KIWCRESC & LSQFIT & MADSEN \\
\hline MAKELA1 & MAKELA2 & MAKELA3 & MAKELA4 & MATRIX2 & MIFFLIN1 \\
\hline MIFFLIN2 & MINMAXBD & MINMAXRB & MISTAKE & POLAK4 & POLAK5 \\
\hline POLAK6 & PRIMAL1 & PRIMALC5 & $\mathrm{QC}$ & ROSENMMX & SIMPLLPA \\
\hline SIMPLLPB & SYNTHES1 & TWOBARS & WOMFLET & ZECEVIC2 & ZECEVIC3 \\
\hline ZECEVIC4 & ZY2 & & & & \\
\hline
\end{tabular}

Tabela C.3: Problemas resolvidos pela versão inexata do Algoritmo 4.3.5 


$\begin{array}{llllll}\text { AIRPORT } & \text { AVGASA } & \text { AVGASB } & \text { CB2 } & \text { CHACONN1 } & \text { CONGIGMZ } \\ \text { DEMYMALO } & \text { DIPIGRI } & \text { EXPFITA } & \text { EXPFITB } & \text { GIGOMEZ1 } & \text { GIGOMEZ2 } \\ \text { HAIFAS } & \text { HIMMELBI } & \text { HS10 } & \text { HS100 } & \text { HS100MOD } & \text { HS108 } \\ \text { HS11 } & \text { HS117 } & \text { HS12 } & \text { HS15 } & \text { HS16 } & \text { HS17 } \\ \text { HS18 } & \text { HS20 } & \text { HS22 } & \text { HS23 } & \text { HS24 } & \text { HS29 } \\ \text { HS31 } & \text { HS33 } & \text { HS34 } & \text { HS35 } & \text { HS35I } & \text { HS35MOD } \\ \text { HS36 } & \text { HS37 } & \text { HS43 } & \text { HS44 } & \text { HS44NEW } & \text { HS65 } \\ \text { HS66 } & \text { HS72 } & \text { HS76 } & \text { HS76I } & \text { HS86 } & \text { HS91 } \\ \text { HS95 } & \text { HS96 } & \text { HS97 } & \text { HS98 } & \text { HUBFIT } & \text { KIWCRESC } \\ \text { LSQFIT } & \text { MADSEN } & \text { MAKELA1 } & \text { MAKELA2 } & \text { MAKELA3 } & \text { MAKELA4 } \\ \text { MATRIX2 } & \text { MIFFLIN1 } & \text { MIFFLIN2 } & \text { MINMAXBD } & \text { MINMAXRB } & \text { MISTAKE } \\ \text { POLAK4 } & \text { POLAK5 } & \text { POLAK6 } & \text { PRIMAL1 } & \text { PRIMALC5 } & \text { ROSENMMX } \\ \text { SIMPLLPA } & \text { SIMPLLPB } & \text { SYNTHES1 } & \text { TWOBARS } & \text { WOMFLET } & \text { ZECEVIC2 } \\ \text { ZECEVIC3 } & \text { ZECEVIC4 } & \text { ZY2 } & & & \end{array}$

Tabela C.4: Problemas usados no teste comparativo do Algoritmo 4.3.5

Apresentamos a seguir as tabelas com indicadores de desempenho das duas versões do Algoritmo 4.3.5. Estas tabelas trazem as seguintes informações:

- A primeira linha refere-se a versão exata do Algoritmo 4.3.5, a segunda a versão inexata.

- A coluna Tempo traz o tempo médio de resolução do problema em segundos. Esta média é obtida de 50 resoluções a partir dos pontos iniciais dados pelo CUTE.

- $\nabla f$ é o número de vezes que o algoritmo calculou o gradiente da função objetivo.

- $\nabla \mathrm{g}$, número de cômputos do gradiente das restrições.

- N. Iter. mede o número de sub-problemas que foram resolvidos para se obter soluções dentro da precisão estipulada.

- V. ótimo é o valor ótimo obtido.

- 75\% Inex. Esta coluna possui 1 caso o critério proposto para aceitar uma solução aproximada dos sub-problemas tenha sido atingindo pelo menos $75 \%$ das vezes. 0 aparece caso contrário. 


\begin{tabular}{|c|c|c|c|c|c|c|}
\hline Problema & Tempo & $\nabla f$ & $\nabla g$ & N. Iter. & V. ótimo & $75 \%$ Inex. \\
\hline AIRPORT & $1.37 \mathrm{E}+01$ & 1702 & 1701 & $\overline{21}$ & $4.795 \mathrm{E}+04$ & $\overline{0}$ \\
\hline AIRPORT & $1.14 \mathrm{E}+01$ & 1380 & 1379 & 21 & $4.795 E+04$ & 0 \\
\hline AVGASA & $1.98 \mathrm{E}-01$ & 238 & 123 & 8 & $-4.632 \mathrm{E}+00$ & 0 \\
\hline AVGASA & $1.49 \mathrm{E}-01$ & 220 & 110 & 8 & $-4.632 \mathrm{E}+00$ & 1 \\
\hline AVGASB & $2.26 \mathrm{E}-01$ & 254 & 154 & 8 & $-4.483 \mathrm{E}+00$ & 0 \\
\hline AVGASB & $1.61 \mathrm{E}-01$ & 186 & 108 & 8 & $-4.483 \mathrm{E}+00$ & 1 \\
\hline CB2 & $1.61 \mathrm{E}-02$ & 70 & 58 & 5 & $1.952 \mathrm{E}+00$ & 0 \\
\hline CB2 & $1.18 \mathrm{E}-02$ & 63 & 51 & 5 & $1.952 \mathrm{E}+00$ & 1 \\
\hline CHACONN1 & $1.86 \mathrm{E}-02$ & 77 & 65 & 5 & $1.952 \mathrm{E}+00$ & 0 \\
\hline CHACONN1 & $1.21 \mathrm{E}-02$ & 61 & 50 & 5 & $1.952 \mathrm{E}+00$ & 1 \\
\hline CONGIGMZ & $9.77 \mathrm{E}-02$ & 169 & 83 & 7 & $2.800 \mathrm{E}+01$ & 0 \\
\hline CONGIGMZ & $1.09 \mathrm{E}-01$ & 238 & 110 & 7 & $2.800 \mathrm{E}+01$ & 0 \\
\hline DEMYMALO & $1.84 \mathrm{E}-02$ & 61 & 48 & 2 & $-3.000 \mathrm{E}+00$ & 0 \\
\hline DEMYMALO & $1.72 \mathrm{E}-02$ & 80 & 67 & 4 & $-3.000 \mathrm{E}+00$ & 1 \\
\hline DIPIGRI & $1.92 \mathrm{E}-01$ & 163 & 76 & 4 & $6.806 \mathrm{E}+02$ & 0 \\
\hline DIPIGRI & $1.80 \mathrm{E}-01$ & 198 & 94 & 4 & $6.806 \mathrm{E}+02$ & 0 \\
\hline EXPFITA & $9.62 \mathrm{E}-01$ & 581 & 157 & 4 & $1.137 \mathrm{E}-03$ & 0 \\
\hline EXPFITA & $8.21 \mathrm{E}-01$ & 495 & 141 & 4 & $1.137 \mathrm{E}-03$ & 1 \\
\hline EXPFITB & $2.22 \mathrm{E}+00$ & 660 & 112 & 9 & $5.019 \mathrm{E}-03$ & 0 \\
\hline EXPFITB & $2.39 \mathrm{E}+00$ & 721 & 114 & 10 & $5.019 \mathrm{E}-03$ & 0 \\
\hline GIGOMEZ1 & $2.16 \mathrm{E}-02$ & 64 & 50 & 2 & $-3.000 \mathrm{E}+00$ & 0 \\
\hline GIGOMEZ1 & $1.69 \mathrm{E}-02$ & 71 & 57 & 3 & $-3.000 \mathrm{E}+00$ & 0 \\
\hline GIGOMEZ2 & $2.14 \mathrm{E}-02$ & 80 & 67 & 5 & $1.952 \mathrm{E}+00$ & 0 \\
\hline GIGOMEZ2 & $1.65 \mathrm{E}-02$ & 72 & 60 & 5 & $1.952 \mathrm{E}+00$ & 1 \\
\hline HAIFAS & $1.54 \mathrm{E}-01$ & 182 & 106 & 6 & $-4.500 \mathrm{E}-01$ & 0 \\
\hline HAIFAS & $9.13 \mathrm{E}-02$ & 229 & 104 & 6 & $-4.500 \mathrm{E}-01$ & 0 \\
\hline HIMMELBI & $7.00 \mathrm{E}+00$ & 1359 & 775 & 3 & $-1.736 \mathrm{E}+03$ & 0 \\
\hline HIMMELBI & $6.11 \mathrm{E}+00$ & 1256 & 715 & 3 & $-1.736 \mathrm{E}+03$ & 0 \\
\hline HS10 & $2.27 \mathrm{E}-02$ & 101 & 101 & 5 & $-1.000 \mathrm{E}+00$ & 0 \\
\hline HS10 & $1.45 \mathrm{E}-02$ & 72 & 72 & 5 & $-1.000 \mathrm{E}+00$ & 1 \\
\hline HS100 & $1.90 \mathrm{E}-01$ & 194 & 92 & 4 & $6.806 \mathrm{E}+02$ & 0 \\
\hline HS100 & $1.88 \mathrm{E}-01$ & 205 & 97 & 4 & $6.806 \mathrm{E}+02$ & 0 \\
\hline HS100MOD & $1.72 \mathrm{E}-01$ & 181 & 48 & 4 & $6.787 \mathrm{E}+02$ & 0 \\
\hline HS100MOD & $1.56 \mathrm{E}-01$ & 138 & 37 & 4 & $6.787 \mathrm{E}+02$ & 0 \\
\hline HS108 & $6.46 \mathrm{E}-02$ & 62 & 54 & 2 & $-8.660 \mathrm{E}-01$ & 0 \\
\hline HS108 & $6.47 \mathrm{E}-02$ & 62 & 54 & 2 & $-8.660 \mathrm{E}-01$ & 0 \\
\hline HS11 & $1.41 \mathrm{E}-02$ & 108 & 108 & 7 & $-8.498 \mathrm{E}+00$ & 0 \\
\hline HS11 & $8.75 \mathrm{E}-03$ & 78 & 78 & 7 & $-8.498 \mathrm{E}+00$ & 1 \\
\hline HS117 & $9.84 \mathrm{E}-01$ & 516 & 475 & 4 & $3.235 \mathrm{E}+01$ & 0 \\
\hline HS117 & $1.10 \mathrm{E}+00$ & 490 & 449 & 4 & $3.235 \mathrm{E}+01$ & 0 \\
\hline HS12 & $1.01 \mathrm{E}-02$ & 75 & 68 & 4 & $-3.000 \mathrm{E}+01$ & 0 \\
\hline HS12 & $7.45 \mathrm{E}-03$ & 71 & 64 & 4 & $-3.000 \mathrm{E}+01$ & 1 \\
\hline
\end{tabular}

Tabela C.5: Comparação entre as versões exata e inexata do Algoritmo 4.3.5 


\begin{tabular}{|c|c|c|c|c|c|c|}
\hline Problema & Tempo & $\nabla f$ & $\nabla g$ & N. Iter. & V. ótimo & $75 \%$ Inex \\
\hline HS15 & $2.94 \mathrm{E}-02$ & 164 & 97 & 17 & $3.065 \mathrm{E}+02$ & 0 \\
\hline HS15 & $2.57 \mathrm{E}-02$ & 121 & 76 & 17 & $3.065 \mathrm{E}+02$ & 1 \\
\hline HS16 & $7.85 \mathrm{E}-03$ & 27 & 23 & 2 & $2.500 \mathrm{E}-01$ & 0 \\
\hline HS16 & $6.75 \mathrm{E}-03$ & 26 & 22 & 2 & $2.500 \mathrm{E}-01$ & 1 \\
\hline HS17 & $1.56 \mathrm{E}-01$ & 1497 & 1496 & 106 & $1.000 \mathrm{E}+00$ & 0 \\
\hline HS17 & $1.43 \mathrm{E}-01$ & 1382 & 1381 & 106 & $1.000 \mathrm{E}+00$ & 0 \\
\hline HS18 & $3.93 \mathrm{E}-02$ & 80 & 34 & 2 & $5.000 \mathrm{E}+00$ & 0 \\
\hline HS18 & $3.38 \mathrm{E}-02$ & 78 & 33 & 2 & $5.000 \mathrm{E}+00$ & 1 \\
\hline HS20 & $1.57 \mathrm{E}-02$ & 110 & 57 & 12 & $3.820 \mathrm{E}+01$ & 0 \\
\hline HS20 & $1.19 \mathrm{E}-02$ & 92 & 50 & 12 & $3.820 \mathrm{E}+01$ & 1 \\
\hline HS22 & $1.01 \mathrm{E}-02$ & 69 & 68 & 7 & $1.000 \mathrm{E}+00$ & 0 \\
\hline HS22 & $7.80 \mathrm{E}-03$ & 62 & 61 & 7 & $1.000 \mathrm{E}+00$ & 1 \\
\hline HS23 & $2.44 \mathrm{E}-02$ & 129 & 82 & 10 & $2.000 \mathrm{E}+00$ & 0 \\
\hline HS23 & $1.91 \mathrm{E}-02$ & 116 & 74 & 10 & $2.000 \mathrm{E}+00$ & 1 \\
\hline HS24 & $4.30 \mathrm{E}-03$ & 35 & 23 & 3 & $0.000 \mathrm{E}+00$ & 0 \\
\hline HS24 & $2.40 \mathrm{E}-03$ & 27 & 18 & 3 & $0.000 \mathrm{E}+00$ & 0 \\
\hline HS29 & $3.89 \mathrm{E}-02$ & 113 & 104 & 4 & $-2.263 \mathrm{E}+01$ & 0 \\
\hline HS29 & $2.80 \mathrm{E}-02$ & 95 & 86 & 4 & $-2.263 \mathrm{E}+01$ & 0 \\
\hline HS31 & $5.10 \mathrm{E}-03$ & 37 & 37 & 4 & $6.000 \mathrm{E}+00$ & 0 \\
\hline HS31 & $4.25 \mathrm{E}-03$ & 37 & 37 & 5 & $6.000 \mathrm{E}+00$ & 1 \\
\hline HS33 & $3.25 \mathrm{E}-03$ & 29 & 16 & 4 & $-4.000 \mathrm{E}+00$ & 0 \\
\hline HS33 & $2.65 \mathrm{E}-03$ & 26 & 14 & 4 & $-4.000 \mathrm{E}+00$ & 1 \\
\hline HS34 & $1.52 \mathrm{E}-02$ & 62 & 62 & 5 & $-8.340 \mathrm{E}-01$ & 0 \\
\hline HS34 & $1.21 \mathrm{E}-02$ & 58 & 58 & 5 & $-8.340 \mathrm{E}-01$ & 1 \\
\hline HS35 & $1.61 \mathrm{E}-02$ & 122 & 122 & 5 & $1.111 \mathrm{E}-01$ & 0 \\
\hline HS35 & $8.10 \mathrm{E}-03$ & 56 & 56 & 5 & $1.111 \mathrm{E}-01$ & 1 \\
\hline HS35I & $1.61 \mathrm{E}-02$ & 122 & 122 & 5 & $1.111 \mathrm{E}-01$ & 0 \\
\hline HS35I & $8.15 \mathrm{E}-03$ & 56 & 56 & 5 & $1.111 \mathrm{E}-01$ & 1 \\
\hline HS35MOD & $9.95 \mathrm{E}-03$ & 78 & 78 & 5 & $2.500 \mathrm{E}-01$ & 0 \\
\hline HS35MOD & $5.70 \mathrm{E}-03$ & 51 & 51 & 6 & $2.500 \mathrm{E}-01$ & 1 \\
\hline HS36 & $4.10 \mathrm{E}-03$ & 68 & 67 & 3 & $-3.300 \mathrm{E}+03$ & 0 \\
\hline HS36 & $2.85 \mathrm{E}-03$ & 44 & 43 & 4 & $-3.300 \mathrm{E}+03$ & 1 \\
\hline HS37 & $1.40 \mathrm{E}-03$ & 15 & 11 & 6 & $0.000 \mathrm{E}+00$ & 0 \\
\hline HS37 & $1.45 \mathrm{E}-03$ & 15 & 11 & 6 & $0.000 \mathrm{E}+00$ & 0 \\
\hline HS43 & $6.18 \mathrm{E}-02$ & 232 & 163 & 11 & $-4.400 \mathrm{E}+01$ & 0 \\
\hline HS43 & $4.55 \mathrm{E}-02$ & 160 & 114 & 11 & $-4.400 \mathrm{E}+01$ & 1 \\
\hline HS44 & $1.37 \mathrm{E}-02$ & 67 & 31 & 6 & $-1.500 \mathrm{E}+01$ & 0 \\
\hline HS44 & $9.85 \mathrm{E}-03$ & 58 & 26 & 6 & $-1.500 \mathrm{E}+01$ & 1 \\
\hline HS44NEW & $1.46 \mathrm{E}-02$ & 88 & 36 & 6 & $-1.500 \mathrm{E}+01$ & 0 \\
\hline HS44NEW & $9.05 \mathrm{E}-03$ & 52 & 23 & 6 & $-1.500 \mathrm{E}+01$ & 1 \\
\hline HS65 & $1.24 \mathrm{E}-02$ & 70 & 62 & 3 & $9.535 \mathrm{E}-01$ & 0 \\
\hline HS65 & $1.19 \mathrm{E}-02$ & 69 & 61 & 3 & $9.535 \mathrm{E}-01$ & 0 \\
\hline
\end{tabular}

Tabela C.6: Comparação entre as versões exata e inexata do Algoritmo 4.3.5 


\begin{tabular}{|c|c|c|c|c|c|c|}
\hline Problema & Tempo & $\nabla f$ & $\nabla g$ & N. Iter. & V. ótimo & $75 \%$ Inex \\
\hline HS66 & $2.47 \mathrm{E}-02$ & 91 & 91 & 6 & $5.182 \mathrm{E}-01$ & 0 \\
\hline HS66 & $2.28 \mathrm{E}-02$ & 90 & 90 & 6 & $5.182 \mathrm{E}-01$ & 0 \\
\hline HS72 & $1.31 \mathrm{E}-01$ & 468 & 468 & 33 & $7.277 \mathrm{E}+02$ & 0 \\
\hline HS72 & $9.28 \mathrm{E}-02$ & 364 & 364 & 33 & $7.277 \mathrm{E}+02$ & 1 \\
\hline HS76 & $1.67 \mathrm{E}-02$ & 74 & 39 & 5 & $-4.682 \mathrm{E}+00$ & 0 \\
\hline HS76 & $1.05 \mathrm{E}-02$ & 57 & 31 & 5 & $-4.682 \mathrm{E}+00$ & 1 \\
\hline HS76I & $1.68 \mathrm{E}-02$ & 74 & 39 & 5 & $-4.682 \mathrm{E}+00$ & 0 \\
\hline HS76I & $1.04 \mathrm{E}-02$ & 57 & 31 & 5 & $-4.682 \mathrm{E}+00$ & 1 \\
\hline HS86 & $1.29 \mathrm{E}-01$ & 313 & 124 & 11 & $-3.235 \mathrm{E}+01$ & 0 \\
\hline HS86 & $8.53 \mathrm{E}-02$ & 198 & 81 & 11 & $-3.235 \mathrm{E}+01$ & 1 \\
\hline HS91 & $4.81 \mathrm{E}+00$ & 339 & 339 & 30 & $1.363 \mathrm{E}+00$ & 0 \\
\hline HS91 & $4.46 \mathrm{E}+00$ & 317 & 317 & 30 & $1.363 \mathrm{E}+00$ & 0 \\
\hline HS95 & $7.40 \mathrm{E}-03$ & 79 & 12 & 2 & $1.562 \mathrm{E}-02$ & 0 \\
\hline HS95 & $7.40 \mathrm{E}-03$ & 82 & 12 & 4 & $1.562 \mathrm{E}-02$ & 1 \\
\hline HS96 & $7.50 \mathrm{E}-03$ & 79 & 12 & 2 & $1.562 \mathrm{E}-02$ & 0 \\
\hline HS96 & $6.95 \mathrm{E}-03$ & 66 & 10 & 3 & $1.562 \mathrm{E}-02$ & 0 \\
\hline HS97 & $4.40 \mathrm{E}-02$ & 72 & 31 & 3 & $4.071 \mathrm{E}+00$ & 0 \\
\hline HS97 & $3.75 \mathrm{E}-02$ & 70 & 30 & 3 & $4.071 \mathrm{E}+00$ & 0 \\
\hline HS98 & $4.40 \mathrm{E}-02$ & 72 & 31 & 3 & $4.071 \mathrm{E}+00$ & 0 \\
\hline HS98 & $3.76 \mathrm{E}-02$ & 70 & 30 & 3 & $4.071 \mathrm{E}+00$ & 0 \\
\hline HUBFIT & $6.85 \mathrm{E}-03$ & 58 & 58 & 6 & $1.689 \mathrm{E}-02$ & 0 \\
\hline HUBFIT & $4.45 \mathrm{E}-03$ & 44 & 44 & 5 & $1.689 \mathrm{E}-02$ & 1 \\
\hline KIWCRESC & $1.11 \mathrm{E}-02$ & 56 & 55 & 5 & $1.069 \mathrm{E}-07$ & 0 \\
\hline KIWCRESC & $8.10 \mathrm{E}-03$ & 52 & 51 & 5 & $1.063 \mathrm{E}-07$ & 1 \\
\hline LSQFIT & $1.31 \mathrm{E}-02$ & 82 & 82 & 7 & $3.379 \mathrm{E}-02$ & 0 \\
\hline LSQFIT & $9.05 \mathrm{E}-03$ & 71 & 71 & 7 & $3.379 \mathrm{E}-02$ & 1 \\
\hline MADSEN & $1.91 \mathrm{E}-02$ & 63 & 41 & 4 & $6.164 \mathrm{E}-01$ & 0 \\
\hline MADSEN & $1.24 \mathrm{E}-02$ & 54 & 34 & 4 & $6.164 \mathrm{E}-01$ & 1 \\
\hline MAKELA1 & $9.75 \mathrm{E}-03$ & 82 & 81 & 8 & $-1.414 \mathrm{E}+00$ & 0 \\
\hline MAKELA1 & 7.10E-03 & 64 & 63 & 8 & $-1.414 \mathrm{E}+00$ & 1 \\
\hline MAKELA2 & $3.59 \mathrm{E}-02$ & 110 & 75 & 4 & $7.200 \mathrm{E}+00$ & 0 \\
\hline MAKELA2 & $2.83 \mathrm{E}-02$ & 107 & 73 & 4 & $7.200 \mathrm{E}+00$ & 0 \\
\hline MAKELA3 & $2.14 \mathrm{E}-01$ & 95 & 89 & 2 & $-4.339 \mathrm{E}-10$ & 0 \\
\hline MAKELA3 & $1.57 \mathrm{E}-01$ & 88 & 82 & 3 & $-1.349 \mathrm{E}-10$ & 0 \\
\hline MAKELA4 & $3.39 \mathrm{E}-01$ & 166 & 96 & 2 & $2.645 \mathrm{E}-10$ & 0 \\
\hline MAKELA4 & $2.65 \mathrm{E}-01$ & 188 & 74 & 17 & $-8.587 \mathrm{E}-11$ & 1 \\
\hline MATRIX2 & $3.30 \mathrm{E}-02$ & 46 & 46 & 2 & $1.297 \mathrm{E}-10$ & 0 \\
\hline MATRIX2 & $1.33 \mathrm{E}-02$ & 34 & 34 & 2 & $3.560 \mathrm{E}-21$ & 0 \\
\hline MIFFLIN1 & $3.90 \mathrm{E}-03$ & 23 & 23 & 2 & $-1.000 \mathrm{E}+00$ & 0 \\
\hline MIFFLIN1 & $5.55 \mathrm{E}-03$ & 37 & 37 & 4 & $-1.000 \mathrm{E}+00$ & 1 \\
\hline MIFFLIN2 & $2.66 \mathrm{E}-02$ & 80 & 77 & 5 & $-1.000 \mathrm{E}+00$ & 0 \\
\hline MIFFLIN2 & $1.84 \mathrm{E}-02$ & 77 & 74 & 5 & $-1.000 \mathrm{E}+00$ & 1 \\
\hline
\end{tabular}

Tabela C.7: Comparação entre as versões exata e inexata do Algoritmo 4.3.5 


\begin{tabular}{|r||r|r|r|r|r|r|}
\hline Problema & Tempo & $\nabla \mathrm{f}$ & $\nabla \mathrm{g}$ & N. Iter. & V. ótimo & $75 \%$ Inex \\
\hline \hline MINMAXBD & $5.03 \mathrm{E}-01$ & 421 & 86 & 3 & $1.157 \mathrm{E}+02$ & 0 \\
MINMAXBD & $4.83 \mathrm{E}-01$ & 405 & 84 & 3 & $1.157 \mathrm{E}+02$ & 0 \\
\hline MINMAXRB & $9.52 \mathrm{E}-02$ & 88 & 82 & 2 & $6.597 \mathrm{E}-10$ & 0 \\
MINMAXRB & $8.42 \mathrm{E}-02$ & 83 & 77 & 2 & $1.836 \mathrm{E}-07$ & 1 \\
\hline MISTAKE & $3.83 \mathrm{E}-01$ & 216 & 142 & 5 & $-1.000 \mathrm{E}+00$ & 0 \\
MISTAKE & $1.06 \mathrm{E}-01$ & 128 & 78 & 5 & $-1.000 \mathrm{E}+00$ & 1 \\
\hline POLAK4 & $1.29 \mathrm{E}-01$ & 564 & 499 & 15 & $-8.548 \mathrm{E}-12$ & 0 \\
POLAK4 & $1.29 \mathrm{E}-01$ & 564 & 499 & 15 & $-8.548 \mathrm{E}-12$ & 0 \\
\hline POLAK5 & $9.25 \mathrm{E}-03$ & 104 & 84 & 2 & $5.000 \mathrm{E}+01$ & 0 \\
POLAK5 & $9.05 \mathrm{E}-03$ & 103 & 83 & 2 & $5.000 \mathrm{E}+01$ & 0 \\
\hline POLAK6 & $1.65 \mathrm{E}-01$ & 190 & 131 & 4 & $-4.400 \mathrm{E}+01$ & 0 \\
POLAK6 & $1.63 \mathrm{E}-01$ & 190 & 132 & 8 & $-4.400 \mathrm{E}+01$ & 0 \\
\hline PRIMAL1 & $2.18 \mathrm{E}+01$ & 1487 & 1209 & 9 & $-3.501 \mathrm{E}-02$ & 0 \\
PRIMAL1 & $2.07 \mathrm{E}+01$ & 1439 & 1152 & 9 & $-3.501 \mathrm{E}-02$ & 0 \\
\hline PRIMALC5 & $3.99 \mathrm{E}+00$ & 1738 & 797 & 39 & $-4.272 \mathrm{E}+02$ & 0 \\
PRIMALC5 & $4.08 \mathrm{E}+00$ & 1678 & 753 & 25 & $-4.272 \mathrm{E}+02$ & 0 \\
\hline ROSENMMX & $1.38 \mathrm{E}-01$ & 205 & 143 & 4 & $-4.400 \mathrm{E}+01$ & 0 \\
ROSENMMX & $1.28 \mathrm{E}-01$ & 169 & 116 & 4 & $-4.400 \mathrm{E}+01$ & 0 \\
\hline SIMPLLPA & $5.40 \mathrm{E}-03$ & 38 & 31 & 4 & $1.000 \mathrm{E}+00$ & 0 \\
SIMPLLPA & $3.95 \mathrm{E}-03$ & 33 & 27 & 4 & $1.000 \mathrm{E}+00$ & 1 \\
\hline SIMPLLPB & $8.45 \mathrm{E}-03$ & 43 & 34 & 3 & $1.100 \mathrm{E}+00$ & 0 \\
SIMPLLPB & $6.80 \mathrm{E}-03$ & 45 & 34 & 4 & $1.100 \mathrm{E}+00$ & 1 \\
\hline SYNTHES1 & $5.80 \mathrm{E}-02$ & 200 & 111 & 11 & $7.593 \mathrm{E}-01$ & 0 \\
SYNTHES1 & $4.70 \mathrm{E}-02$ & 178 & 99 & 11 & $7.593 \mathrm{E}-01$ & 0 \\
\hline TWOBARS & $1.32 \mathrm{E}-02$ & 80 & 50 & 8 & $1.509 \mathrm{E}+00$ & 0 \\
TWOBARS & $8.40 \mathrm{E}-03$ & 65 & 41 & 8 & $1.509 \mathrm{E}+00$ & 1 \\
\hline WOMFLET & $1.37 \mathrm{E}-01$ & 140 & 122 & 3 & $-4.533 \mathrm{E}-09$ & 0 \\
WOMFLET & $1.44 \mathrm{E}-01$ & 140 & 122 & 3 & $-8.577 \mathrm{E}-09$ & 0 \\
\hline ZECEVIC2 & $7.45 \mathrm{E}-03$ & 37 & 28 & 3 & $-4.125 \mathrm{E}+00$ & 0 \\
ZECEVIC2 & $4.35 \mathrm{E}-03$ & 31 & 23 & 3 & $-4.125 \mathrm{E}+00$ & 1 \\
\hline ZECEVIC3 & $1.30 \mathrm{E}-02$ & 108 & 59 & 7 & $9.731 \mathrm{E}+01$ & 0 \\
ZECEVIC3 & $7.75 \mathrm{E}-03$ & 67 & 38 & 7 & $9.731 \mathrm{E}+01$ & 1 \\
\hline ZECEVIC4 & $7.40 \mathrm{E}-03$ & 50 & 28 & 5 & $7.558 \mathrm{E}+00$ & 0 \\
ZECEVIC4 & $5.60 \mathrm{E}-03$ & 44 & 25 & 5 & $7.558 \mathrm{E}+00$ & 1 \\
\hline ZY2 & $3.25 \mathrm{E}-03$ & 29 & 16 & 4 & $2.000 \mathrm{E}+00$ & 0 \\
ZY2 & $2.80 \mathrm{E}-03$ & 27 & 15 & 4 & $2.000 \mathrm{E}+00$ & 1 \\
\hline \hline & & & & & & \\
\hline
\end{tabular}

Tabela C.8: Comparação entre as versões exata e inexata do Algoritmo 4.3.5 


\section{C.2 Método Híbrido Projeção-Lagrangiano Aumen- tado Proximal}

Apresentamos a seguir as tabelas com os problemas resolvidos por cada uma das versões de nossa implementação do método híbrido. Como o conjunto inicial de 130 testes é o mesmo da seção anterior ele não será repetido.

$\begin{array}{llllll}\text { AIRPORT } & \text { AVGASA } & \text { AVGASB } & \text { CANTILVR } & \text { CB2 } & \text { CHACONN1 } \\ \text { CONGIGMZ } & \text { DEMYMALO } & \text { DIPIGRI } & \text { GIGOMEZ1 } & \text { GIGOMEZ2 } & \text { HAIFAS } \\ \text { HALDMADS } & \text { HIMMELB } & \text { HIMMELP2 } & \text { HIMMELP } & \text { HIMMELP4 } & \text { HIMMELP5 } \\ \text { HIMMELP6 } & \text { HS10 } & \text { HS100 } & \text { HS100MOD } & \text { HS108 } & \text { HS11 } \\ \text { HS113 } & \text { HS12 } & \text { HS15 } & \text { HS16 } & \text { HS17 } & \text { HS18 } \\ \text { HS20 } & \text { HS21 } & \text { HS21MOD } & \text { HS22 } & \text { HS23 } & \text { HS24 } \\ \text { HS29 } & \text { HS30 } & \text { HS31 } & \text { HS33 } & \text { HS34 } & \text { HS35 } \\ \text { HS35I } & \text { HS35MOD } & \text { HS36 } & \text { HS37 } & \text { HS43 } & \text { HS44 } \\ \text { HS44NEW } & \text { HS65 } & \text { HS66 } & \text { HS72 } & \text { HS76 } & \text { HS76I } \\ \text { HS86 } & \text { HS88 } & \text { HS90 } & \text { HS91 } & \text { HS92 } & \text { HS95 } \\ \text { HS96 } & \text { HS97 } & \text { HS98 } & \text { HUBFIT } & \text { KIWCRESC } & \text { LSQFIT } \\ \text { MADSEN } & \text { MAKELA1 } & \text { MAKELA2 } & \text { MAKELA3 } & \text { MAKELA4 } & \text { MATRIX2 } \\ \text { MIFFLIN1 } & \text { MIFFLIN2 } & \text { MINMAXBD } & \text { MINMAXRB } & \text { MISTAKE } & \text { POLAK5 } \\ \text { POLAK6 } & \text { PRIMAL1 } & \text { QC } & \text { ROSENMMX } & \text { SIMPLLPA } & \text { SIMPLLPB } \\ \text { SYNTHES1 } & \text { TWOBARS } & \text { WOMFLET } & \text { ZECEVIC2 } & \text { ZECEVIC3 } & \text { ZECEVIC4 } \\ \text { ZY2 } & & & & & \end{array}$

Tabela C.9: Problemas resolvidos pela versão exata do algoritmo Híbrido

$\begin{array}{llllll}\text { AVGASA } & \text { AVGASB } & \text { CANTILVR } & \text { CB2 } & \text { CB3 } & \text { CHACONN1 } \\ \text { CHACONN2 } & \text { CONGIGMZ } & \text { DEMYMALO } & \text { DIPIGRI } & \text { GIGOMEZ1 } & \text { GIGOMEZ2 } \\ \text { GIGOMEZ3 } & \text { HAIFAS } & \text { HALDMADS } & \text { HIMMELP2 } & \text { HIMMELP3 } & \text { HIMMELP4 } \\ \text { HIMMELP5 } & \text { HIMMELP6 } & \text { HS10 } & \text { HS100 } & \text { HS100MOD } & \text { HS108 } \\ \text { HS11 } & \text { HS113 } & \text { HS117 } & \text { HS12 } & \text { HS15 } & \text { HS16 } \\ \text { HS17 } & \text { HS18 } & \text { HS19 } & \text { HS20 } & \text { HS21 } & \text { HS21MOD } \\ \text { HS22 } & \text { HS23 } & \text { HS24 } & \text { HS29 } & \text { HS30 } & \text { HS31 } \\ \text { HS33 } & \text { HS34 } & \text { HS35 } & \text { HS35I } & \text { HS35MOD } & \text { HS36 } \\ \text { HS37 } & \text { HS43 } & \text { HS44 } & \text { HS44NEW } & \text { HS65 } & \text { HS66 } \\ \text { HS72 } & \text { HS76 } & \text { HS76I } & \text { HS86 } & \text { HS88 } & \text { HS89 } \\ \text { HS90 } & \text { HS91 } & \text { HS92 } & \text { HS93 } & \text { HS97 } & \text { HS98 } \\ \text { HUBFIT } & \text { KIWCRESC } & \text { LSQFIT } & \text { MADSEN } & \text { MAKELA1 } & \text { MAKELA2 } \\ \text { MATRIX2 } & \text { MIFFLIN1 } & \text { MIFFLIN2 } & \text { MINMAXRB } & \text { MISTAKE } & \text { POLAK5 } \\ \text { POLAK6 } & \text { PRIMAL1 } & \text { QC } & \text { ROSENMMX } & \text { SIMPLLPA } & \text { SIMPLLPB } \\ \text { SYNTHES1 } & \text { TWOBARS } & \text { WOMFLET } & \text { ZECEVIC2 } & \text { ZECEVIC3 } & \text { ZECEVIC4 } \\ \text { ZY2 } & & & & & \end{array}$

Tabela C.10: Problemas resolvidos pela versão inexata do algoritmo Híbrido 


$\begin{array}{llllll}\text { AVGASA } & \text { AVGASB } & \text { CANTILVR } & \text { CB2 } & \text { CHACONN1 } & \text { CONGIGMZ } \\ \text { DEMYMALO } & \text { DIPIGRI } & \text { GIGOMEZ1 } & \text { GIGOMEZ2 } & \text { HAIFAS } & \text { HALDMADS } \\ \text { HIMMELP5 } & \text { HIMMELP6 } & \text { HS10 } & \text { HS100 } & \text { HS100MOD } & \text { HS108 } \\ \text { HS11 } & \text { HS113 } & \text { HS12 } & \text { HS15 } & \text { HS16 } & \text { HS17 } \\ \text { HS18 } & \text { HS20 } & \text { HS22 } & \text { HS23 } & \text { HS24 } & \text { HS29 } \\ \text { HS31 } & \text { HS33 } & \text { HS34 } & \text { HS35 } & \text { HS35I } & \text { HS35MOD } \\ \text { HS36 } & \text { HS43 } & \text { HS44 } & \text { HS44NEW } & \text { HS65 } & \text { HS66 } \\ \text { HS72 } & \text { HS76 } & \text { HS76I } & \text { HS86 } & \text { HS88 } & \text { HS90 } \\ \text { HS91 } & \text { HS92 } & \text { HS97 } & \text { HS98 } & \text { HUBFIT } & \text { KIWCRESC } \\ \text { LSQFIT } & \text { MADSEN } & \text { MAKELA1 } & \text { MAKELA2 } & \text { MATRIX2 } & \text { MIFFLIN1 } \\ \text { MIFFLIN2 } & \text { MINMAXRB } & \text { MISTAKE } & \text { POLAK5 } & \text { POLAK6 } & \text { PRIMAL1 } \\ \text { ROSENMMX } & \text { SIMPLLPA } & \text { SIMPLLPB } & \text { SYNTHES1 } & \text { TWOBARS } & \text { WOMFLET } \\ \text { ZECEVIC2 } & \text { ZECEVIC3 } & \text { ZECEVIC4 } & \text { ZY2 } & & \end{array}$

Tabela C.11: Problemas usados no teste comparativo do algoritmo Híbrido

Apresentamos a seguir as tabelas com indicadores de desempenho das duas versões do método híbrido. Mais uma vez, estas tabelas trazem as seguintes informações:

- A primeira linha refere-se a versão exata do método híbrido projeção-lagrangiano aumentado proximal, a segunda a versão inexata.

- A coluna Tempo traz o tempo médio de resolução do problema em segundos. Esta média é obtida de 50 resoluções a partir dos pontos iniciais dados pelo CUTE.

- $\nabla f$ é o número de vezes que o algoritmo calculou o gradiente da função objetivo.

- $\nabla \mathrm{g}$, número de cômputos do gradiente das restrições.

- N. Iter. mede o número de sub-problemas que foram resolvidos para se obter soluções dentro da precisão estipulada.

- V. ótimo é o valor ótimo obtido.

- 75\% Inex. Esta coluna possui 1 caso o critério proposto para aceitar uma solução aproximada dos sub-problemas tenha sido atingindo pelo menos $75 \%$ das vezes. 0 aparece caso contrário. 


\begin{tabular}{|c|c|c|c|c|c|c|}
\hline Problema & Tempo & $\nabla f$ & $\nabla \mathrm{g}$ & N. Iter. & V. ótimo & $75 \%$ Inex \\
\hline AVGASA & $2.25 \mathrm{E}-01$ & 285 & 122 & 8 & $-4.632 \mathrm{E}+00$ & 0 \\
\hline AVGASA & $3.23 \mathrm{E}-01$ & 283 & 110 & 8 & $-4.632 \mathrm{E}+00$ & 0 \\
\hline AVGASB & $1.69 \mathrm{E}-01$ & 213 & 97 & 8 & $-4.483 \mathrm{E}+00$ & 0 \\
\hline AVGASB & $3.68 \mathrm{E}-01$ & 383 & 159 & 8 & $-4.483 \mathrm{E}+00$ & 0 \\
\hline CANTILVR & $5.02 \mathrm{E}-02$ & 244 & 225 & 19 & $1.340 \mathrm{E}+00$ & 0 \\
\hline CANTILVR & $1.72 \mathrm{E}-02$ & 184 & 165 & 20 & $1.340 \mathrm{E}+00$ & 1 \\
\hline CB2 & $1.80 \mathrm{E}-02$ & 108 & 75 & 8 & $1.952 \mathrm{E}+00$ & 0 \\
\hline CB2 & $1.48 \mathrm{E}-02$ & 106 & 71 & 8 & $1.952 \mathrm{E}+00$ & 1 \\
\hline CHACONN1 & $1.50 \mathrm{E}-02$ & 128 & 90 & 10 & $1.952 \mathrm{E}+00$ & 0 \\
\hline CHACONN1 & $1.16 \mathrm{E}-02$ & 104 & 73 & 10 & $1.952 \mathrm{E}+00$ & 1 \\
\hline CONGIGMZ & $1.51 \mathrm{E}-01$ & 384 & 174 & 12 & $2.800 \mathrm{E}+01$ & 0 \\
\hline CONGIGMZ & $3.23 \mathrm{E}-01$ & 1065 & 460 & 16 & $2.800 \mathrm{E}+01$ & 0 \\
\hline DEMYMALO & $2.92 \mathrm{E}-02$ & 157 & 135 & 7 & $-3.000 \mathrm{E}+00$ & 0 \\
\hline DEMYMALO & $2.04 \mathrm{E}-02$ & 136 & 101 & 8 & $-3.000 \mathrm{E}+00$ & 1 \\
\hline DIPIGRI & $2.43 \mathrm{E}-01$ & 264 & 124 & 5 & $6.806 \mathrm{E}+02$ & 0 \\
\hline DIPIGRI & $1.57 \mathrm{E}-01$ & 179 & 74 & 4 & $6.806 \mathrm{E}+02$ & 1 \\
\hline GIGOMEZ1 & $5.40 \mathrm{E}-02$ & 193 & 135 & 7 & $-3.000 \mathrm{E}+00$ & 0 \\
\hline GIGOMEZ1 & $2.76 \mathrm{E}-02$ & 169 & 103 & 8 & $-3.000 \mathrm{E}+00$ & 1 \\
\hline GIGOMEZ2 & $2.44 \mathrm{E}-02$ & 109 & 70 & 8 & $1.952 \mathrm{E}+00$ & 0 \\
\hline GIGOMEZ2 & $1.20 \mathrm{E}-02$ & 94 & 60 & 7 & $1.952 \mathrm{E}+00$ & 1 \\
\hline HAIFAS & $1.74 \mathrm{E}-01$ & 347 & 154 & 8 & $-4.500 \mathrm{E}-01$ & 0 \\
\hline HAIFAS & $2.30 \mathrm{E}-01$ & 444 & 178 & 8 & $-4.500 \mathrm{E}-01$ & 1 \\
\hline HALDMADS & $8.10 \mathrm{E}-01$ & 790 & 326 & 17 & $1.222 \mathrm{E}-04$ & 0 \\
\hline HALDMADS & $1.15 \mathrm{E}+00$ & 998 & 326 & 17 & $1.222 \mathrm{E}-04$ & 1 \\
\hline HIMMELP5 & $4.66 \mathrm{E}-02$ & 263 & 57 & 7 & $-5.901 \mathrm{E}+01$ & 0 \\
\hline HIMMELP5 & $1.86 \mathrm{E}-02$ & 209 & 35 & 8 & $-5.901 \mathrm{E}+01$ & 1 \\
\hline HIMMELP6 & $4.76 \mathrm{E}-02$ & 263 & 34 & 7 & $-5.901 \mathrm{E}+01$ & 0 \\
\hline HIMMELP6 & $3.54 \mathrm{E}-02$ & 178 & 19 & 8 & $-5.901 \mathrm{E}+01$ & 1 \\
\hline HS10 & $1.90 \mathrm{E}-02$ & 129 & 125 & 9 & $-1.000 \mathrm{E}+00$ & 0 \\
\hline HS10 & $9.00 \mathrm{E}-03$ & 102 & 100 & 9 & $-1.000 \mathrm{E}+00$ & 1 \\
\hline HS100 & $2.40 \mathrm{E}-01$ & 225 & 104 & 5 & $6.806 \mathrm{E}+02$ & 0 \\
\hline HS100 & $2.94 \mathrm{E}-01$ & 269 & 119 & 5 & $6.806 \mathrm{E}+02$ & 1 \\
\hline HS100MOD & $2.06 \mathrm{E}-01$ & 214 & 52 & 5 & $6.787 \mathrm{E}+02$ & 0 \\
\hline HS100MOD & $2.88 \mathrm{E}-01$ & 236 & 57 & 5 & $6.787 \mathrm{E}+02$ & 1 \\
\hline HS108 & $1.42 \mathrm{E}-01$ & 186 & 92 & 7 & $-8.660 \mathrm{E}-01$ & 0 \\
\hline HS108 & $2.87 \mathrm{E}-01$ & 235 & 128 & 7 & $-8.660 \mathrm{E}-01$ & 0 \\
\hline HS11 & $8.40 \mathrm{E}-03$ & 77 & 74 & 7 & $-8.498 \mathrm{E}+00$ & 0 \\
\hline HS11 & $4.80 \mathrm{E}-03$ & 70 & 66 & 7 & $-8.498 \mathrm{E}+00$ & 1 \\
\hline HS113 & $5.31 \mathrm{E}-01$ & 480 & 331 & 6 & $2.431 \mathrm{E}+01$ & 0 \\
\hline HS113 & $5.69 \mathrm{E}-01$ & 526 & 352 & 8 & $2.431 \mathrm{E}+01$ & 0 \\
\hline HS12 & $1.58 \mathrm{E}-02$ & 153 & 136 & 5 & $-3.000 \mathrm{E}+01$ & 0 \\
\hline HS12 & $8.60 \mathrm{E}-03$ & 89 & 75 & 5 & $-3.000 \mathrm{E}+01$ & 1 \\
\hline
\end{tabular}

Tabela C.12: Comparação entre as versões exata e inexata do algoritmo Híbrido 


\begin{tabular}{|c|c|c|c|c|c|c|}
\hline Problema & Tempo & $\nabla f$ & $\nabla \mathrm{g}$ & N. Iter. & V. ótimo & $75 \%$ Inex \\
\hline HS15 & $4.14 \mathrm{E}-02$ & 122 & 68 & 17 & $3.065 \mathrm{E}+02$ & 0 \\
\hline HS15 & $4.44 \mathrm{E}-02$ & 284 & 144 & 17 & $3.065 \mathrm{E}+02$ & 0 \\
\hline HS16 & $1.76 \mathrm{E}-02$ & 53 & 22 & 5 & 2.500E-01 & 0 \\
\hline HS16 & $9.60 \mathrm{E}-03$ & 73 & 19 & 6 & $2.500 \mathrm{E}-01$ & 0 \\
\hline HS17 & $3.22 \mathrm{E}-02$ & 129 & 114 & 10 & $1.000 \mathrm{E}+00$ & 0 \\
\hline HS17 & $1.38 \mathrm{E}-02$ & 114 & 99 & 10 & $1.000 \mathrm{E}+00$ & 1 \\
\hline HS18 & $7.56 \mathrm{E}-02$ & 1024 & 508 & 96 & $5.000 \mathrm{E}+00$ & 0 \\
\hline HS18 & $6.62 \mathrm{E}-02$ & 1087 & 527 & 99 & $5.000 \mathrm{E}+00$ & 1 \\
\hline HS20 & $1.78 \mathrm{E}-02$ & 158 & 57 & 12 & $3.820 \mathrm{E}+01$ & 0 \\
\hline HS20 & $1.36 \mathrm{E}-02$ & 137 & 69 & 12 & $4.020 \mathrm{E}+01$ & 0 \\
\hline HS22 & $6.00 \mathrm{E}-03$ & 64 & 58 & 7 & $1.000 \mathrm{E}+00$ & 0 \\
\hline HS22 & $3.60 \mathrm{E}-03$ & 57 & 51 & 7 & $1.000 \mathrm{E}+00$ & 1 \\
\hline HS23 & $1.76 \mathrm{E}-02$ & 138 & 60 & 9 & $2.000 \mathrm{E}+00$ & 0 \\
\hline HS23 & $1.00 \mathrm{E}-02$ & 123 & 52 & 9 & $2.000 \mathrm{E}+00$ & 1 \\
\hline HS24 & $9.20 \mathrm{E}-03$ & 93 & 46 & 9 & $-1.000 \mathrm{E}+00$ & 0 \\
\hline HS24 & $5.20 \mathrm{E}-03$ & 83 & 42 & 9 & $-1.000 \mathrm{E}+00$ & 1 \\
\hline HS29 & $4.14 \mathrm{E}-02$ & 166 & 148 & 6 & $-2.263 \mathrm{E}+01$ & 0 \\
\hline HS29 & $2.90 \mathrm{E}-02$ & 141 & 118 & 6 & $-2.263 \mathrm{E}+01$ & 1 \\
\hline HS31 & $7.40 \mathrm{E}-03$ & 72 & 69 & 8 & $6.000 \mathrm{E}+00$ & 0 \\
\hline HS31 & $5.40 \mathrm{E}-03$ & 72 & 69 & 8 & $6.000 \mathrm{E}+00$ & 0 \\
\hline HS33 & $4.40 \mathrm{E}-03$ & 58 & 23 & 7 & $-4.000 \mathrm{E}+00$ & 0 \\
\hline HS33 & $4.60 \mathrm{E}-03$ & 62 & 25 & 7 & $-4.000 \mathrm{E}+00$ & 0 \\
\hline HS34 & $7.22 \mathrm{E}-02$ & 439 & 436 & 28 & $-8.340 \mathrm{E}-01$ & 0 \\
\hline HS34 & $1.04 \mathrm{E}-01$ & 654 & 567 & 28 & $-8.340 \mathrm{E}-01$ & 1 \\
\hline HS35 & $1.48 \mathrm{E}-02$ & 128 & 125 & 8 & $1.111 \mathrm{E}-01$ & 0 \\
\hline HS35 & $6.20 \mathrm{E}-03$ & 75 & 72 & 8 & $1.111 \mathrm{E}-01$ & 1 \\
\hline HS35I & $1.46 \mathrm{E}-02$ & 128 & 125 & 8 & $1.111 \mathrm{E}-01$ & 0 \\
\hline HS35I & $6.20 \mathrm{E}-03$ & 75 & 72 & 8 & $1.111 \mathrm{E}-01$ & 1 \\
\hline HS35MOD & $1.08 \mathrm{E}-02$ & 116 & 42 & 13 & $2.500 \mathrm{E}-01$ & 0 \\
\hline HS35MOD & $1.06 \mathrm{E}-02$ & 114 & 47 & 13 & $2.500 \mathrm{E}-01$ & 0 \\
\hline HS36 & $1.80 \mathrm{E}-03$ & 33 & 30 & 8 & $-3.300 \mathrm{E}+03$ & 0 \\
\hline HS36 & $5.40 \mathrm{E}-03$ & 75 & 67 & 8 & $-3.300 \mathrm{E}+03$ & 0 \\
\hline HS43 & $5.20 \mathrm{E}-02$ & 185 & 118 & 8 & $-4.400 \mathrm{E}+01$ & 0 \\
\hline HS43 & $3.28 \mathrm{E}-02$ & 129 & 83 & 8 & $-4.400 \mathrm{E}+01$ & 1 \\
\hline HS44 & $1.26 \mathrm{E}-02$ & 83 & 23 & 7 & $-1.500 \mathrm{E}+01$ & 0 \\
\hline HS44 & $1.28 \mathrm{E}-02$ & 87 & 24 & 7 & $-1.500 \mathrm{E}+01$ & 0 \\
\hline HS44NEW & $1.24 \mathrm{E}-02$ & 93 & 27 & 7 & $-1.500 \mathrm{E}+01$ & 0 \\
\hline HS44NEW & $3.74 \mathrm{E}-02$ & 195 & 50 & 7 & $-1.500 \mathrm{E}+01$ & 0 \\
\hline HS65 & $3.78 \mathrm{E}-02$ & 190 & 136 & 10 & $9.535 \mathrm{E}-01$ & 0 \\
\hline HS65 & $2.94 \mathrm{E}-02$ & 182 & 134 & 10 & $9.535 \mathrm{E}-01$ & 1 \\
\hline HS66 & $7.24 \mathrm{E}-02$ & 335 & 333 & 23 & $5.182 \mathrm{E}-01$ & 0 \\
\hline HS66 & $4.00 \mathrm{E}-02$ & 294 & 289 & 23 & $5.182 \mathrm{E}-01$ & 1 \\
\hline
\end{tabular}

Tabela C.13: Comparação entre as versões exata e inexata do algoritmo Híbrido 


\begin{tabular}{|c|c|c|c|c|c|c|}
\hline Problema & Tempo & $\nabla f$ & $\nabla \mathrm{g}$ & N. Iter. & V. ótimo & $75 \%$ Inex \\
\hline " HS72 & $8.72 \mathrm{E}-02$ & 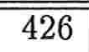 & $\overline{426}$ & 30 & $\overline{77.277 \mathrm{E}+02}$ & $\begin{array}{ll} & 0\end{array}$ \\
\hline HS72 & $7.56 \mathrm{E}-02$ & 365 & 361 & 30 & $7.277 \mathrm{E}+02$ & 1 \\
\hline HS76 & $1.38 \mathrm{E}-02$ & 91 & 26 & 8 & $-4.682 \mathrm{E}+00$ & 0 \\
\hline HS76 & $6.54 \mathrm{E}-02$ & 192 & 50 & 8 & $-4.682 \mathrm{E}+00$ & 0 \\
\hline HS76I & $1.40 \mathrm{E}-02$ & 91 & 26 & 8 & $-4.682 \mathrm{E}+00$ & 0 \\
\hline HS76I & $6.56 \mathrm{E}-02$ & 192 & 50 & 8 & $-4.682 \mathrm{E}+00$ & 0 \\
\hline HS86 & $9.80 \mathrm{E}-02$ & 230 & 75 & 10 & $-3.235 \mathrm{E}+01$ & 0 \\
\hline HS86 & $7.90 \mathrm{E}-02$ & 198 & 67 & 10 & $-3.235 \mathrm{E}+01$ & 1 \\
\hline HS88 & $1.31 \mathrm{E}+00$ & 360 & 360 & 31 & $1.363 \mathrm{E}+00$ & 0 \\
\hline HS88 & $1.79 \mathrm{E}+00$ & 485 & 485 & 34 & $1.363 \mathrm{E}+00$ & 0 \\
\hline HS90 & $2.82 \mathrm{E}+00$ & 319 & 319 & 31 & $1.363 \mathrm{E}+00$ & 0 \\
\hline HS90 & $8.00 \mathrm{E}+00$ & 893 & 887 & 34 & $1.363 \mathrm{E}+00$ & 0 \\
\hline HS91 & $5.67 \mathrm{E}+00$ & 444 & 444 & 31 & $1.363 \mathrm{E}+00$ & 0 \\
\hline HS91 & $1.76 \mathrm{E}+01$ & 1348 & 1346 & 39 & $1.363 \mathrm{E}+00$ & 0 \\
\hline HS92 & $6.05 \mathrm{E}+00$ & 358 & 358 & 31 & $1.363 \mathrm{E}+00$ & 0 \\
\hline HS92 & $1.53 \mathrm{E}+01$ & 894 & 885 & 34 & $1.363 \mathrm{E}+00$ & 0 \\
\hline HS97 & $1.66 \mathrm{E}-02$ & 67 & 15 & 3 & $4.071 \mathrm{E}+00$ & 0 \\
\hline HS97 & $4.06 \mathrm{E}-02$ & 115 & 27 & 3 & $4.071 \mathrm{E}+00$ & 0 \\
\hline HS98 & $1.68 \mathrm{E}-02$ & 67 & 15 & 3 & $4.071 \mathrm{E}+00$ & 0 \\
\hline HS98 & $4.04 \mathrm{E}-02$ & 115 & 27 & 3 & $4.071 \mathrm{E}+00$ & 0 \\
\hline HUBFIT & $1.18 \mathrm{E}-02$ & 101 & 70 & 10 & $1.689 \mathrm{E}-02$ & 0 \\
\hline HUBFIT & $6.60 \mathrm{E}-03$ & 87 & 64 & 10 & $1.689 \mathrm{E}-02$ & 1 \\
\hline KIWCRESC & $2.46 \mathrm{E}-02$ & 128 & 121 & 8 & $-1.387 \mathrm{E}-07$ & 0 \\
\hline KIWCRESC & $1.02 \mathrm{E}-02$ & 109 & 100 & 9 & $-4.891 \mathrm{E}-08$ & 1 \\
\hline LSQFIT & $9.00 \mathrm{E}-03$ & 91 & 67 & 10 & $3.379 \mathrm{E}-02$ & 0 \\
\hline LSQFIT & $5.40 \mathrm{E}-03$ & 83 & 66 & 10 & $3.379 \mathrm{E}-02$ & 1 \\
\hline MADSEN & $3.02 \mathrm{E}-02$ & 123 & 43 & 7 & $6.164 \mathrm{E}-01$ & 0 \\
\hline MADSEN & $1.36 \mathrm{E}-02$ & 98 & 33 & 7 & $6.164 \mathrm{E}-01$ & 1 \\
\hline MAKELA1 & $8.00 \mathrm{E}-03$ & 69 & 57 & 7 & $-1.414 \mathrm{E}+00$ & 0 \\
\hline MAKELA1 & $3.40 \mathrm{E}-03$ & 55 & 44 & 7 & $-1.414 \mathrm{E}+00$ & 1 \\
\hline MAKELA2 & $3.68 \mathrm{E}-02$ & 155 & 100 & 10 & $7.200 \mathrm{E}+00$ & 0 \\
\hline MAKELA2 & $3.32 \mathrm{E}-02$ & 177 & 111 & 10 & $7.200 \mathrm{E}+00$ & 1 \\
\hline MATRIX2 & $1.16 \mathrm{E}-02$ & 75 & 37 & 8 & $6.454 \mathrm{E}-15$ & 0 \\
\hline MATRIX2 & $1.62 \mathrm{E}-02$ & 96 & 48 & 8 & $8.594 \mathrm{E}-14$ & 0 \\
\hline MIFFLIN1 & $1.22 \mathrm{E}-02$ & 95 & 90 & 8 & $-1.000 \mathrm{E}+00$ & 0 \\
\hline MIFFLIN1 & $5.60 \mathrm{E}-03$ & 76 & 72 & 8 & $-1.000 \mathrm{E}+00$ & 1 \\
\hline MIFFLIN2 & $2.96 \mathrm{E}-02$ & 147 & 124 & 8 & $-1.000 \mathrm{E}+00$ & 0 \\
\hline MIFFLIN2 & $3.04 \mathrm{E}-02$ & 175 & 148 & 8 & $-1.000 \mathrm{E}+00$ & 1 \\
\hline MINMAXRB & $1.39 \mathrm{E}-01$ & 310 & 207 & 9 & $-6.011 \mathrm{E}-12$ & 0 \\
\hline MINMAXRB & $1.14 \mathrm{E}-01$ & 267 & 192 & 9 & $4.449 \mathrm{E}-09$ & 1 \\
\hline MISTAKE & $1.78 \mathrm{E}-01$ & 214 & 68 & 7 & $-1.000 \mathrm{E}+00$ & 0 \\
\hline MISTAKE & 4.82E-02 & 134 & 42 & 7 & $-1.000 \mathrm{E}+00$ & 1 \\
\hline
\end{tabular}

Tabela C.14: Comparação entre as versões exata e inexata do algoritmo Híbrido 


\begin{tabular}{|r||r|r|r|r|r|r|}
\hline Problema & Tempo & $\nabla \mathrm{f}$ & $\nabla \mathbf{g}$ & N. Iter. & V. ótimo & $75 \%$ Inex \\
\hline \hline POLAK5 & $7.62 \mathrm{E}-02$ & 224 & 205 & 10 & $5.000 \mathrm{E}+01$ & 0 \\
POLAK5 & $4.08 \mathrm{E}-02$ & 322 & 220 & 10 & $5.000 \mathrm{E}+01$ & 1 \\
\hline POLAK6 & $5.16 \mathrm{E}-01$ & 753 & 233 & 11 & $-4.400 \mathrm{E}+01$ & 0 \\
POLAK6 & $3.58 \mathrm{E}-01$ & 741 & 269 & 15 & $-4.400 \mathrm{E}+01$ & 1 \\
\hline PRIMAL1 & $1.11 \mathrm{E}+01$ & 966 & 708 & 6 & $-3.501 \mathrm{E}-02$ & 0 \\
PRIMAL1 & $7.30 \mathrm{E}+00$ & 622 & 453 & 6 & $-3.501 \mathrm{E}-02$ & 1 \\
\hline ROSENMMX & $2.25 \mathrm{E}-01$ & 601 & 197 & 12 & $-4.400 \mathrm{E}+01$ & 0 \\
ROSENMMX & $3.89 \mathrm{E}-01$ & 765 & 274 & 15 & $-4.400 \mathrm{E}+01$ & 1 \\
\hline SIMPLLPA & $2.40 \mathrm{E}-03$ & 41 & 24 & 8 & $1.000 \mathrm{E}+00$ & 0 \\
SIMPLLPA & $3.20 \mathrm{E}-03$ & 57 & 34 & 9 & $1.000 \mathrm{E}+00$ & 0 \\
\hline SIMPLLPB & $6.00 \mathrm{E}-03$ & 69 & 45 & 9 & $1.100 \mathrm{E}+00$ & 0 \\
SIMPLLPB & $6.40 \mathrm{E}-03$ & 82 & 53 & 9 & $1.100 \mathrm{E}+00$ & 1 \\
\hline SYNTHES1 & $4.78 \mathrm{E}-02$ & 380 & 189 & 10 & $7.593 \mathrm{E}-01$ & 0 \\
SYNTHES1 & $1.15 \mathrm{E}-01$ & 292 & 125 & 10 & $7.593 \mathrm{E}-01$ & 0 \\
\hline TWOBARS & $1.02 \mathrm{E}-02$ & 118 & 62 & 8 & $1.509 \mathrm{E}+00$ & 0 \\
TWOBARS & $4.60 \mathrm{E}-03$ & 63 & 32 & 8 & $1.509 \mathrm{E}+00$ & 1 \\
\hline WOMFLET & $2.69 \mathrm{E}-01$ & 452 & 372 & 14 & $1.212 \mathrm{E}-11$ & 0 \\
WOMFLET & $2.46 \mathrm{E}-02$ & 162 & 53 & 10 & $6.050 \mathrm{E}+00$ & 1 \\
\hline ZECEVIC2 & $9.00 \mathrm{E}-03$ & 80 & 40 & 8 & $-4.125 \mathrm{E}+00$ & 0 \\
ZECEVIC2 & $4.20 \mathrm{E}-03$ & 64 & 32 & 8 & $-4.125 \mathrm{E}+00$ & 1 \\
\hline ZECEVIC3 & $7.80 \mathrm{E}-03$ & 74 & 36 & 7 & $9.731 \mathrm{E}+01$ & 0 \\
ZECEVIC3 & $4.60 \mathrm{E}-03$ & 66 & 32 & 7 & $9.731 \mathrm{E}+01$ & 1 \\
\hline ZECEVIC4 & $6.20 \mathrm{E}-03$ & 108 & 53 & 6 & $7.558 \mathrm{E}+00$ & 0 \\
ZECEVIC4 & $3.60 \mathrm{E}-03$ & 58 & 27 & 7 & $7.558 \mathrm{E}+00$ & 1 \\
\hline ZY2 & $4.60 \mathrm{E}-03$ & 57 & 22 & 7 & $2.000 \mathrm{E}+00$ & 0 \\
ZY2 & $8.80 \mathrm{E}-03$ & 90 & 38 & 7 & $2.000 \mathrm{E}+00$ & 0 \\
\hline
\end{tabular}

Tabela C.15: Comparação entre as versões exata e inexata do algoritmo Híbrido 


\section{Referências Bibliográficas}

[1] A. Auslender, R. Cominetti, and M. Haddou. Asymptotic analysis for penalty and barrier methods in convex and linear programming. Mathematics of Operations Research, 22(1):43-62, 1997.

[2] A. Auslender and M. Haddou. An interior-proximal methods for convex linearly constrained problems and its extension to variational problems. Mathematical Programming, 71:77-100, 1995.

[3] A. Auslender and M. Teboulle. Lagrangian duality and related multiplier methods for variational inequality problems. SIAM J. Optim., 10(4):1097-1115, 2000.

[4] A. Auslender, M. Teboulle, and S. Ben-Tiba. Interior proximal and multiplier methods based on second order homogeneous kernels. Mathematics of Operations Research, 24:645-668, 1999.

[5] A. Auslender, M. Teboulle, and S. Ben-Tiba. A logarithmic-quadratic proximal method for variational inequalities. Computational Optimization and Applications, 12:31-40, 1999 .

[6] A. BenTal and M. Zibulevsky. Penalty/barrier multiplier methods for convex programming problems. SIAM Journal on Optimization, 7(2):347-366, 1997.

[7] D. Bertsekas. Nonlinear Programming. Athena Scientific, 1995.

[8] D. Bertsekas. Constrained Optimization and Lagrange Multipliers. Athena Scientific, 1996.

[9] J. R. Birge, L. Qi, and Z. Wei. A general approach to convergence properties of some methods for nonsmooth convex optimization. Applied Mathematics and Optimization, 38:141-158, 1998. 
[10] I. Bongartz, A. R. Conn, N. Gold, and Ph.L. Toint. CUTE: Constrained and Unconstrained Testing Enviroment. ACM Transactions on Mathematical Software, 21:121-160, 1995.

[11] L Bregman. The relaxation method of finding the common points of convex sets and its application to the solution of problems in convex programming. USSR Computational Mathematics and Mathematical Physics, 7:200-217, 1967.

[12] H. Brézis and A. Haraux. Image d'une somme d'operateurs monotones et applications. Israel Journal of Mathematics, 23(2):165-186, 1976.

[13] R. Burachik and B. Svaiter. $\epsilon$-enlargements of maximal monotone operators in Banach spaces. Set valued Analysis, 7(2):117-132, 1999.

[14] R. S. Burachik. Generalized Proximal Point Methods for the Variational Inequality Problem. PhD thesis, Insituto de Matemática Pura e Aplicada, IMPA, 1995.

[15] R. H. Byrd, P. Lu, J. Nocedal, and C. Y. Zhu. A limited memory algorithm for bound constrained optimization. SIAM Journal on Scientific Computing, 16(5):1190-1208, 1995.

[16] Y. Censor and J Zenios. The proximal minimization algorithms with D-functions. Jounal of Optimization: Theory and Applications, 73:451-464, 1992.

[17] Chunhui Chen and O. L. Mangasarian. A class of smoothing functions for nonlinear and mixed complementarity problems. Comput. Optim. Appl., 5(2):97-138, 1996.

[18] G. Chen and M. Teboulle. A convergence analysis of proximal-like minimization algorithms using Bregman functions. SIAM Journal on Optimization, 3:538-543, 1993.

[19] J. Eckstein. Nonlinear proximal point algorithms using Bregman functions, with applications to convex programming. Mathematics of Operations Research, 18:202226, 1993.

[20] J. Eckstein. Approximate iterations in Bregman-function-based proximal algorithms. Mathematical Programming, 83:113-124, 1998.

[21] J. Gauvin. A necessary and sufficient condition to have bounded multipliers in nonconvex programming. Mathematical Programming, 12:136-138, 1977.

[22] M.R. Hestenes. Multiplier and gradient methods. Jornal on Optimization: Theory and Applications, 4:303-320, 1969. 
[23] J.-B. Hiriart-Urruty and C. Lemaréchal. Convex analysis and minimization algorithms. I, Fundamentals. Springer-Verlag, Berlin, 1993.

[24] J.-B. Hiriart-Urruty and C. Lemaréchal. Convex analysis and minimization algorithms. II, Advanced theory and bundle methods. Springer-Verlag, Berlin, 1993.

[25] C. Humes and P. Silva. Strict convex regularizations, proximal point and augmented lagrangians. RAIRO Operations Research, 34(3):283-303, 2000.

[26] C. Humes, P. Silva, and B. F. Svaiter. An inexact hybrid projection-proximal augmented lagrangian algorithm. In Proceedings of the Thirty Sixth Annual Allerton Conference on Communication, Control and Computing, pages 450-459, 1998.

[27] A. Iusem. Métodos de Ponto Proximal em Otimização. Instituto de Matemática Pura e Aplicada - CNPq, 1995. Livro do $20^{\circ}$ Colóquio Brasileiro de Matemática.

[28] A. Iusem, M. Teboulle, and B. Svaiter. Entropy-like proximal methods in covex programming. Mathematics of Operations Research, 19(4):790-814, Novembro 1994.

[29] O. L. Mangasarian. Nonlinear Programming. McGraw-Hill Book Company, New York, 1969.

[30] V.H. Nguyen, J.J. Strodiot, and R. Mifflin. Conditions to have bounded multipliers in locally Lipschitz programming. Mathematical Programming, 16(3):100-106, 1978.

[31] M. Pappalardo. Error bounds for generalized Lagrange multipliers in locally Lipschitz programming. Journal on Optimization: Theory and Applications, 73(1):205210, 1992.

[32] T. Pennanen. Multiplier methods for monotone inclusions. comunicação pessoal, 1998.

[33] L. Polak. Optimization: Algorithms and Consistent Approximations. Springer Verlag, 1997.

[34] M.J.D. Powell. Optimization, chapter A method for nonlinear constraints in minimization problems, pages 283-298. Academic Press, New York, 1969. Edited by Fletcher, R.

[35] Burachick. R. and A. Iusem. A generalized proximal point algorithm for the variational inequality problem in a Hilbert space. SIAM Journal on Optimization, 8(1):197-216, 1998. 
[36] R. T. Rockafellar. Extension of Fenchel's duality theorem for convex functions. Duke Mathematical Journal, 33:81-89, 1966.

[37] R. T. Rockafellar. Convex Analysis. Princeton University Press, 1970.

[38] R. T. Rockafellar. Conjugate Duality and Optimization. Society for Industrial and Applied Mathematics, 1974.

[39] R. T. Rockafellar. Augmented lagrangians and applications of the proximal point algorithm in convex programming. Mathematics of Operations Research, 1:97-116, 1976.

[40] R. T. Rockafellar. Monotone operators and the proximal point algorithm. SIAM Journal on Control and Optimization, 14:887-898, Agosto 1976.

[41] R. T. Rockafellar and R. J.-B. Wets. Variational analysis, volume 317 of Grundlehren der Mathematischen Wissenschaften [Fundamental Principles of Mathematical Sciences]. Springer-Verlag, Berlin, 1998.

[42] P. Silva, J. Eckstein, and C. Humes. Rescaling and stepsize selection in proximal methods using generalized distances. Technical Report RRR 35-99, Rutgers Center for Operations Research, 1999. Submetido.

[43] M. Solodov and B. Svaiter. A hybrid projection-proximal point algorithm. Jounal of Convex Analysis, 6(1), 1999.

[44] M. Solodov and B. Svaiter. An inexact hybrid generalized proximal point algorithm and some new results in the theory of Bregman functions. Mathematics of Operations Research, 25(2):214-230, 2000.

[45] M. Teboulle. Entropic proximal methods with aplications to nonlinear programming. Mathematics of Operations Research, 17:670-690, 1992. 